TRANSACTIONS OF THE

AMERICAN MATHEMATICAL SOCIETY

Volume 358, Number 2, Pages 509-551

S 0002-9947(05)03858-4

Article electronically published on September 9, 2005

\title{
STEINBERG SYMBOLS MODULO THE TRACE CLASS, HOLONOMY, AND LIMIT THEOREMS FOR TOEPLITZ DETERMINANTS
}

\author{
RICHARD W. CAREY AND JOEL D. PINCUS
}

Abstract. Suppose that $\phi=\psi z^{\gamma}$ where $\gamma \in Z_{+}$and $\psi \in \operatorname{Lip}_{\beta}, \frac{1}{2}<\beta<1$, and the Toeplitz operator $T_{\psi}$ is invertible. Let $D_{n}\left(T_{\phi}\right)$ be the determinant of the Toeplitz matrix $\left(\left(\hat{\phi}_{i, j}\right)\right)=\left(\left(\hat{\phi}_{i-j}\right)\right), \quad 0 \leq i, j \leq n$, where $\hat{\phi}_{k}=$ $\frac{1}{2 \pi} \int_{0}^{2 \pi} \phi(\theta) e^{-i k \theta} d \theta$. Let $P_{n}$ be the orthogonal projection onto $\operatorname{ker} S^{* n+1}=$ $\bigvee\left\{1, e^{i \theta}, e^{2 i \theta}, \ldots, e^{i n \theta}\right\}$, where $S=T_{z}$; set $Q_{n}=1-P_{n}$, let $H_{\omega}$ denote the Hankel operator associated to $\omega$, and set $\tilde{\omega}(t)=\omega\left(\frac{1}{t}\right)$ for $t \in \mathbb{T}$. For the Wiener-Hopf factorization $\psi=f \bar{g}$ where $f, g$ and $\frac{1}{f}, \frac{1}{g} \in \operatorname{Lip}_{\beta} \cap H^{\infty}(\mathbb{T}), \frac{1}{2}<$ $\beta<1$, put $E(\psi)=\exp \sum_{k=1}^{\infty} k(\log f)_{k}(\log \bar{g})_{-k}, G(\psi)=\exp (\log \psi)_{0}$.

Theorem A. $\quad D_{n}\left(T_{\phi}\right)=(-1)^{(n+1) \gamma} G(\psi)^{n+1} E(\psi) G\left(\frac{\bar{g}}{f}\right)^{\gamma}$

$$
\cdot \operatorname{det}\left(\left(T_{\frac{f}{\bar{g}} z^{n+1}} \cdot\left[1-H_{\frac{\bar{g}}{f}} Q_{n-\gamma} H_{\left(\frac{f}{\bar{g}}\right)^{\gamma}}\right]^{-1} z^{\alpha-1}, z^{\tau-1}\right)\right)_{\gamma \times \gamma} \cdot\left[1+O\left(n^{1-2 \beta}\right)\right] .
$$

Let $H^{2}(\mathbb{T})=\mathcal{X}+\mathcal{Y}$ be a decomposition into $T_{\phi} T_{\phi^{-1}}$ invariant subspaces, $\mathcal{X}=\bigcap_{n=1}^{\infty} \operatorname{ran}\left(T_{\phi} T_{\phi^{-1}}\right)^{n}$ and $\mathcal{Y}=\bigcup_{n=1}^{\infty} \operatorname{ker}\left(T_{\phi} T_{\phi^{-1}}\right)^{n}$, so that $T_{\phi} T_{\phi^{-1}}$ restricted to $\mathcal{X}$ is invertible, $\mathcal{Y}$ is finite dimensional, and $T_{\phi} T_{\phi^{-1}}$ restricted to $\mathcal{Y}$ is nilpotent. Let $\left\{w_{\alpha}\right\}_{1}^{\gamma}$ be the basis $\left\{T_{f} z^{\alpha}\right\}_{0}^{\gamma-1}$ for the null space of $T_{\phi} T_{\phi^{-1}}$, and let $u_{\alpha}$ be the top vector in a Jordan root vector chain of length $m_{\alpha}+1$ lying over $(-1)^{m_{\alpha}} w_{\alpha}$, i.e., $\left(T_{\phi} T_{\phi}-1\right)^{m_{\alpha}} u_{\alpha}=(-1)^{m_{\alpha}} w_{\alpha}$ where $m_{\alpha}=\max \left\{m \in Z_{+}: \exists x \text { so that }\left(T_{\phi} T_{\phi^{-1}}\right)^{m} x=w_{\alpha}\right\}^{-1}$.

Theorem B. $E(\psi) G\left(\frac{\bar{g}}{f}\right)^{\gamma}=\frac{\left.\prod_{\lambda \in \sigma\left(T_{\phi} T_{\phi}-1\right.}\right) \backslash\{0\}}{} \lambda=\left(\bar{g} \cup f \times \frac{\bar{g}}{f} \cup z^{\gamma}\right)(\mathbb{T})$, the holonomy of a Deligne bundle with connection defined by the factorization $\phi=f \bar{g} z^{\gamma}$.

Note that the generalizations of the Szegö limit theorem for $D_{n}\left(T_{\phi}\right)$ which have appeared in the literature with 1 instead of $\left[1-H_{\frac{\bar{g}}{f}} Q_{n-\gamma} H_{\left(\frac{f}{g}\right)^{-}}\right]^{-1}$ have the defect that the limit of $\frac{D_{n}\left(T_{\phi}\right)}{(-1)^{(n+1) \gamma G(\psi)^{n+1} \operatorname{det}\left(T_{\frac{f}{g}} z^{n+1} z^{\alpha-1}, z^{\tau-1}\right)}}$ does not exist in general. An example is given with $D_{n}\left(T_{\phi}\right) \stackrel{\frac{f}{g}}{\neq} 0$ yet $D_{\gamma-1}\left(T_{\frac{f}{g}} z^{n+1}\right)=0$ for infinitely many $n$.

Received by the editors January 23, 2004.

2000 Mathematics Subject Classification. Primary 47A55, 47B30, 47A53, 47B35, 46L87, $19 \mathrm{C} 20$.

(C)2005 American Mathematical Society Reverts to public domain 28 years from publication 


\section{INTRODUCTION}

Jacobi's theorem on the conjugate minors of the adjugate matrix formed from the cofactors of $D_{n}\left(T_{\phi}\right)$ has been the main tool of previous attempts to generalize the classical strong Szegö limit theorem. But Toeplitz operators are defined by an algebraic relation with the shift, $S^{* n+1} T_{\phi} S^{n+1}=T_{\phi}$, and it is natural instead to probe the implications of the Toeplitz property algebraically. The result gives, for the first time to our knowledge, a full extension of the Szegö theorem to the case where wind $(\phi, 0) \neq 0$,

$$
\begin{aligned}
& \lim _{n \rightarrow \infty} \frac{D_{n}\left(T_{\phi}\right)}{(-1)^{(n+1) \gamma} G(\psi)^{n+1} \cdot \operatorname{det}\left(\left(T_{\frac{f}{\bar{g}} z^{n+1}} \cdot\left[1-H_{\frac{\bar{g}}{f}} Q_{n-\gamma} H_{\left(\frac{f}{\bar{g}}\right)}^{-1} z^{\alpha-1}, z^{\tau-1}\right)\right)_{\gamma \times \gamma}\right.} \\
& \quad=E(\psi) G\left(\frac{\bar{g}}{f}\right)^{\gamma}=\frac{\prod_{\lambda \in \sigma\left(T_{\phi} T_{\phi}-1\right) \backslash\{0\}} \lambda}{\operatorname{det}\left(u_{\alpha}, T_{\frac{1}{g}} z^{\tau-1}\right)_{\gamma \times \gamma}},
\end{aligned}
$$

and shows that the limit splits into two parts, a "torsion", $\prod_{\lambda \in \sigma\left(T_{\phi} T_{\phi}-1\right) \backslash\{0\}} \lambda$, the product of the non-zero eigenvalues of $T_{\phi} T_{\phi^{-1}}$ and a denominator reflecting the Jordan chain structure of the finite dimensional nilpotent operator $T_{\phi} T_{\phi^{-1}}$ acting on $\mathcal{Y}$.

The methods we employ have wider implications. In Orthogonal polynomials and periodic recurrence relations, perturbation vectors are related to linear systems on Riemann surfaces to give the orthogonal polynomials corresponding to periodic Jacobi matrices.

Steinberg symbols. The relative algebraic $K$ group $K_{1}\left(\mathcal{L}(H), \mathcal{L}^{1}(H)\right)$ may be identified with the quotient group of the invertible elements of $\mathcal{L}(H)$ of the form $1+J$ where $J$ is in $\mathcal{L}^{1}(H)$, by the commutator subgroup $\left\{1+\mathcal{L}^{1}(H), \mathcal{L}(H)\right\}$ generated by elements of the form $\{1+J, A\} \equiv(1+J) A(1+J)^{-1} A^{-1}$ where $A$ and $1+$ $J$ are invertible and $J$ is in $\mathcal{L}^{1}(H)$. If $J$ is trace class and $1+J$ is invertible, then write $[1+J]_{1}$ for the corresponding element in $K_{1}\left(\mathcal{L}(H), \mathcal{L}^{1}(H)\right)$. Every element in $K_{1}\left(\mathcal{L}(H), \mathcal{L}^{1}(H)\right)$ has such a representation. The map det is a group homomorphism of the invertible operators of the form $1+J$, where $J \in \mathcal{L}^{1}(H)$, onto

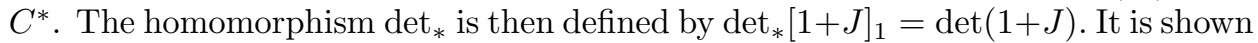
in [1] that the map $\operatorname{det}_{*}$ is the projection onto the first factor in the isomorphism $K_{1}\left(\mathcal{L}(H), \mathcal{L}^{1}(H)\right) \cong C^{*} \oplus V$ in which $V$ has uncountable linear dimension.

Furthermore, there is a connecting map $\partial$, so that there is a homomorphism to the non-zero complex numbers $C^{*}$ given by the composition

$$
\operatorname{det}_{*} \circ \partial: K_{2}\left(\mathcal{L}(H) / \mathcal{L}^{1}(H)\right) \rightarrow C^{*},
$$

where, for invertible elements $\alpha, \beta \in \mathcal{L}(H) / \mathcal{L}^{1}(H)$ which happen to commute, there is the Steinberg symbol

$$
\{\alpha, \beta\} \in K_{2}\left(\mathcal{L}(H) / \mathcal{L}^{1}(H)\right) \stackrel{\partial}{\longrightarrow} K_{1}\left(\mathcal{L}(H) ; \mathcal{L}^{1}(H)\right) \cong C^{*} \oplus V .
$$

Let $R, S$, and $T$ denote regularizers for $A, B$ and $A B$ respectively. Thus $R A-$ $I, A R-I \in \mathcal{L}^{1}(H)$, etc. The elements $a, b \in \mathcal{L}(H) / \mathcal{L}^{1}(H)$ are lifted to $A, B$ and $a^{-1}, b^{-1},(a b)^{-1}$ are lifted to $R, S$ and $T$. 
The definition of Steinberg symbols leads to (see [15])

$$
\begin{aligned}
\partial\{a, b\}=\left[\left(\begin{array}{cc}
I & A \\
0 & I
\end{array}\right) \cdot\left(\begin{array}{cc}
I & 0 \\
-R & I
\end{array}\right) \cdot\left(\begin{array}{ll}
I & A \\
0 & I
\end{array}\right) \cdot\left(\begin{array}{cc}
I & -I \\
0 & I
\end{array}\right) \cdot\left(\begin{array}{ll}
I & 0 \\
I & I
\end{array}\right)\right. \\
\cdot\left(\begin{array}{cc}
I & -I \\
0 & I
\end{array}\right) \cdot\left(\begin{array}{cc}
I & B \\
0 & I
\end{array}\right) \cdot\left(\begin{array}{cc}
I & 0 \\
-S & I
\end{array}\right) \cdot\left(\begin{array}{cc}
I & B \\
0 & I
\end{array}\right) \\
\left.\cdot\left(\begin{array}{cc}
I & -A B \\
0 & I
\end{array}\right) \cdot\left(\begin{array}{cc}
I & 0 \\
T & I
\end{array}\right) \cdot\left(\begin{array}{cc}
I & -A B \\
0 & I
\end{array}\right)\right] \bmod \mathcal{L}^{1}(H),
\end{aligned}
$$

where there is latitude in the choice of regularizers, since different regularizers lead to the same class, and therefore define liftings with the same determinant.

Thus, for example, when $T_{\phi}$ is invertible, take $A=T_{\phi}, B=S^{*}$ so that $A B=$ $T_{\phi} S^{*}$ with regularizers $T=S T_{\phi}^{-1}, R=T_{\phi}{ }^{-1}$. Then, by substitution as above,

$$
\begin{gathered}
\partial\left\{T_{\phi}+\mathcal{L}^{1}(H), S^{*}+\mathcal{L}^{1}(H)\right\} \\
=\left[\left(\begin{array}{cc}
I & T_{\phi} \\
0 & I
\end{array}\right) \cdot\left(\begin{array}{cc}
I & 0 \\
-T_{\phi}^{-1} & I
\end{array}\right) \cdot\left(\begin{array}{cc}
I & T_{\phi} \\
0 & I
\end{array}\right) \cdot\left(\begin{array}{cc}
I & -I \\
0 & I
\end{array}\right) \cdot\left(\begin{array}{cc}
I & 0 \\
I & I
\end{array}\right) \cdot\left(\begin{array}{cc}
I & -I \\
0 & I
\end{array}\right)\right. \\
\left.\cdot\left(\begin{array}{cc}
I & S^{*} \\
0 & I
\end{array}\right) \cdot\left(\begin{array}{cc}
I & 0 \\
-S & I
\end{array}\right) \cdot\left(\begin{array}{cc}
I & S^{*} \\
0 & I
\end{array}\right) \cdot\left(\begin{array}{cc}
I & -T_{\phi} S^{*} \\
0 & I
\end{array}\right) \cdot\left(\begin{array}{cc}
I & 0 \\
S T_{\phi}^{-1} & I
\end{array}\right) \cdot\left(\begin{array}{cc}
I & -T_{\phi} S^{*} \\
0 & I
\end{array}\right)\right] .
\end{gathered}
$$

If we multiply these twelve factors together and use $S S^{*}=1-P_{0}$, we get

$$
\operatorname{det}_{*} \circ \partial\left\{T_{\phi}+\mathcal{L}^{1}(H), S^{*}+\mathcal{L}^{1}(H)\right\}=\operatorname{det}\left(T_{\phi}{ }^{-1} S T_{\phi} S^{*}+T_{\phi}{ }^{-1} P_{0}\right) .
$$

Thus,

$$
\begin{gathered}
\frac{\operatorname{det}\left(P_{0} T_{\phi} P_{0}\right)}{\operatorname{det}_{*} \circ \partial\left\{T_{\phi}+\mathcal{L}^{1}(H), S+\mathcal{L}^{1}(H)\right\}} \\
=\operatorname{det}\left(P_{0} T_{\phi} P_{0}\right) \cdot \operatorname{det}_{*} \circ \partial\left\{T_{\phi}+\mathcal{L}^{1}(H), S^{*}+\mathcal{L}^{1}(H)\right\} \\
=\operatorname{det}\left(P_{0}^{\perp}+P_{0} T_{\phi}\right) \cdot \operatorname{det}\left(T_{\phi}{ }^{-1} S T_{\phi} S^{*}+T_{\phi}{ }^{-1} P_{0}\right) \\
=\operatorname{det}\left(P_{0}^{\perp} T_{\phi}{ }^{-1} S T_{\phi} S^{*}+P_{0}^{\perp} T_{\phi}{ }^{-1} P_{0}+P_{0}\right)=\operatorname{det}\left(P_{0}^{\perp} T_{\phi}{ }^{-1} S T_{\phi} S^{*}+P_{0}\right) \\
=\operatorname{det}\left(P_{0}^{\perp} T_{\phi}{ }^{-1} S T_{\phi} S^{*}+1-S S^{*}\right)=\operatorname{det}\left(1+\left(P_{0}^{\perp} T_{\phi}{ }^{-1} S T_{\phi}-S\right) S^{*}\right) \\
=\operatorname{det}\left(1+S^{*}\left(P_{0}^{\perp} T_{\phi}{ }^{-1} S T_{\phi}-S\right)\right)=\operatorname{det}\left(1+S^{*}\left(T_{\phi}{ }^{-1} S T_{\phi}-S\right)\right)=\operatorname{det}\left(S^{*} T_{\phi}{ }^{-1} S T_{\phi}\right) .
\end{gathered}
$$

The "same" calculation carried out with $S^{*}$ replaced by $S^{* n+1}$ and $P_{0}$ replaced by $P_{n}$ gives

$$
\frac{\operatorname{det}\left(P_{n} T_{\phi} P_{n}\right)}{\operatorname{det}_{*} \circ \partial\left\{T_{\phi}+\mathcal{L}^{1}(H), S^{n+1}+\mathcal{L}^{1}(H)\right\}}=\operatorname{det}\left(S^{* n+1} T_{\phi}{ }^{-1} S^{n+1} T_{\phi}\right) .
$$

Proposition 1.1 (Equation (35) of [15]). If $\phi \in \mathcal{L}^{\infty}(0,2 \pi)$ with $T_{\phi}$ Fredholm, injective, and $D_{n}(\phi) \neq 0$, then

$$
\frac{D_{n}\left(T_{\phi}\right)}{\operatorname{det}_{*} \circ \partial\left\{T_{\phi}+\mathcal{L}^{1}(H), S^{n+1}+\mathcal{L}^{1}(H)\right\}}=\operatorname{det}\left(T_{\phi} S^{* n+1} R\left(T_{\phi}\right) S^{n+1}+P\left(T_{\bar{\phi}}\right) S^{n+1}\right) .
$$

The Steinberg symbol determinant in the denominator makes sense when $T_{\phi}$ is Fredholm and not invertible and as already noted can be expressed explicitly. It is shown in $\S 1.2$, that if $T_{\psi}$ is invertible, then $\operatorname{det}_{*} \circ \partial\left\{T_{\psi z^{\gamma}}+\mathcal{L}^{1}(H), S^{n+1}+\mathcal{L}^{1}(H)\right\}=$ $\mathbf{G}(\phi)^{n+1}=G(\psi)^{n+1} \cdot(-1)^{(n+1) \gamma}$. 
Thus, when $T_{\phi}$ is invertible and $\gamma=0$, since $\mathbf{G}(\phi)=G(\psi)$, the geometric mean, the proposition becomes the very special case proved above:

$$
\frac{D_{n}\left(T_{\phi}\right)}{G(\phi)^{n+1}}=\operatorname{det}\left(S^{* n+1} T_{\phi}^{-1} S^{n+1} T_{\phi}\right) .
$$

This immediately gives the strong Szegö limit theorem

$$
\lim _{n \rightarrow \infty} \frac{D_{n}\left(T_{\phi}\right)}{G(\phi)^{n+1}}=\operatorname{det}\left(T_{\phi} T_{\phi^{-1}}\right) .
$$

Proposition 1.1 was proved in [15] in order to treat the case where wind $(\phi, 0) \neq 0$. Nevertheless, since there have now been a number of papers (published subsequent to [15]) giving variant proofs of a formula of A. Borodin and A. Okunkov [9]:

$$
\operatorname{det}\left(P_{n} T_{\phi} P_{n}\right)=G(\phi)^{n+1} \frac{\operatorname{det}\left[1-Q_{n} H_{\frac{\bar{g}}{f}} H_{\frac{\tilde{f}}{\tilde{g}}} Q_{n}\right]}{\operatorname{det}\left[1-H_{\frac{\bar{g}}{f}} H_{\frac{\tilde{f}}{\tilde{g}}}\right]},
$$

for the case of invertible $T_{\phi}, \phi=f \bar{g}$, we note the following:

\section{Remark 1.1. The Borodin-Okunkov formula is equivalent to the special case $(*)$ of Proposition 1.1.}

This is immediate.

Since $T_{a b}=T_{a} T_{b}+H_{a} H_{\tilde{b}}$, and $T_{\phi}$ is invertible, we have, with $\tilde{b}(t) \equiv b(\bar{t})$ for $t \in \mathbb{T}$, and $\langle\alpha, \beta\rangle=\alpha \beta \alpha^{-1} \beta^{-1}$ denoting the multiplicative commutator of the operators $\alpha$ and $\beta$,

$$
\begin{gathered}
\operatorname{det}\left(T_{\phi} S^{* n+1} T_{\phi}^{-1} S^{n+1}\right)=\operatorname{det}\left(T_{\bar{g}} T_{f} S^{* n+1} T_{f-1} T_{\bar{g}-1} S^{n+1}\right) \\
=\operatorname{det}\left(T_{f}\left\langle T_{f-1}, T_{\bar{g}}\right\rangle T_{\bar{g}} S^{* n+1} T_{f-1} T_{\bar{g}^{-1}} S^{n+1}\right) \\
=\operatorname{det}\left(\left\langle T_{f}, T_{\bar{g}}\right\rangle\right)\left(S^{* n+1} T_{\bar{g}} T_{f^{-1}} T_{\bar{g}^{-1}} T_{f} S^{n+1}\right) \\
=\frac{\operatorname{det}\left(S^{* n+1} T_{\frac{\bar{g}}{f}} T_{\frac{f}{\bar{g}}} S^{n+1}\right)}{\operatorname{det}\left(\left\langle T_{\bar{g}}, T_{f^{-1}}\right\rangle\right)}=\frac{\operatorname{det}\left[1-Q_{n} H_{\frac{\bar{g}}{f}} H_{\frac{\tilde{f}}{\tilde{g}}} Q_{n}\right]}{\operatorname{det}\left[1-H_{\frac{\bar{g}}{f}} H_{\frac{\tilde{f}}{\tilde{g}}}\right]} .
\end{gathered}
$$

However, the goal here is to describe a more comprehensive framework for wind $(\phi, 0) \neq 0$. Thus, in 15 . we introduced perturbation vectors $\sigma_{A, B}$ associated to a pair of Fredholm operators $A, B$ with $A-B$ in the trace ideal. These non-zero vectors are a substitute for the perturbation determinants studied by Aronszjan, Weinstein, M. G. Krein, and many others. In the special case where $A$ is the identity, $\sigma_{A, B}$ splits and the scalar part of these perturbation vectors amounts to taking the product of the non-zero eigenvalues, while the vector itself comes from a section of an appropriate bundle.

To create this object the authors proved that there exists a trivialization of the pullback $i_{M}^{*}(\wp)$ of a determinant bundle, given by a section, $\sigma$, which they called the perturbation section. Let $\mathcal{L}(H)$ be the algebra of all bounded linear operators on a Hilbert space $H$. Let $\mathcal{L}^{1}(\mathcal{H})$ denote the ideal of compact operators $T$ with trace $|T|<\infty$. Let $\mathcal{F}$ denote the Fredholm operators on $H$. Let $\mathcal{Q} \rightarrow \mathcal{F}$ be the Quillen det bundle. Form $\mathcal{Q}+\mathcal{Q}^{*}$ as a bundle over $\mathcal{F} \times \mathcal{F}$. Let $\wp=\operatorname{det}\left(\mathcal{Q}+\mathcal{Q}^{*}\right)$ be the determinant bundle. Let $M \equiv\left\{(A, B):(A, B) \in \mathcal{F} \times \mathcal{F}\right.$ and $\left.A-B \in \mathcal{L}^{1}(H)\right\}$ with $i_{M}: M \rightarrow \mathcal{F} \times \mathcal{F}$ the inclusion map.

The perturbation vector $\sigma_{A, B}$ is the value of the section at $(A, B) \in M$. 
When wind $(\phi, 0)$ is not zero and the normalized Szegö determinant $\frac{D_{n}\left(T_{\phi}\right.}{G(\phi)^{n+1}}$ converges to zero, the perturbation vector $\sigma_{1, T_{\phi} S *^{n+1}} R\left(T_{\phi}\right) S^{n+1}$ gives a replacement 1 because

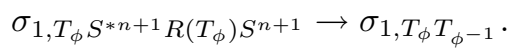

The analysis of the convergence of this sequence of perturbation vectors in combination with Proposition 1.1 will be our basic study. A review of the perturbation vector construction and some of their properties is given in $\S 2$.

We will see in $\S 2$ that if $1-X \in \mathcal{L}^{1}$, then

$$
\left\|\sigma_{1, X}\right\|=\frac{\prod_{\lambda \in \sigma(X) \backslash\{0\}}|\lambda|}{\left|\operatorname{det}\left(u_{i}, y_{j}\right)\right|}=\prod_{s_{\alpha}(X) \neq 0} \mathbf{s}_{\alpha}(X),
$$

where $\left\{y_{j}\right\}$ is any orthonormal basis for $\operatorname{ker} X^{*},\left\{u_{i}\right\}$ is any set of maximal root vectors relative to any orthonormal basis of $\operatorname{ker} X$ and $\left\{\mathbf{s}_{\alpha}(X)\right\}$ are the singular values of $X$. For example this implies that $d d^{c} \prod_{\mathbf{s}_{\alpha} \neq 0} \mathbf{s}_{\alpha}\left(T_{z-\lambda} T_{(z-\lambda)^{-1}}\right)=$ $d d^{c}\left\|\sigma_{1, T_{z-\lambda}} T_{(z-\lambda)^{-1}}\right\|=-\frac{1}{\left(1-|\lambda|^{2}\right)^{2}} d \lambda \wedge d \bar{\lambda}$ is the Gaussian curvature of the Hermitian bundle whose fibre at the point $\lambda$ is $\operatorname{ker} T_{z-\lambda}{ }^{*}$.

The perturbation vector version of Proposition 1.1. Proposition 1.1 is the scalar part of a vector result proved in [15].

The fact that the ratio

$$
\frac{D_{n}\left(T_{\phi}\right)}{\operatorname{det}\left(T_{\phi} S^{*}{ }^{n+1} R\left(T_{\phi}\right) S^{n+1}+P\left(T_{\bar{\phi}}\right) S^{n+1}\right)}=\operatorname{det}_{*} \circ \partial\left\{T_{\phi}+\mathcal{L}^{1}(H), S^{n+1}+\mathcal{L}^{1}(H)\right\}
$$

depends only on the cosets of $T_{\phi}$ and the shift $S$ modulo the trace ideal is part of a statement made in terms of perturbation vectors:

For $\gamma=0$,

$$
\begin{aligned}
\sigma_{S^{* n+1}, T_{\phi} S^{* n+1} T_{\phi}-1} & =\frac{\operatorname{det}\left(T_{\phi} S^{* n+1} T_{\phi}^{-1} S^{n+1}\right)}{D_{n}\left(T_{\phi}\right)} \rho_{n}^{*} \otimes T_{\phi} \rho_{n} \\
& =\frac{\rho_{n}^{*} \otimes T_{\phi} \rho_{n}}{\operatorname{det}_{*} \circ \partial\left\{T_{\phi}+\mathcal{L}^{1}(H), S^{n+1}+\mathcal{L}^{1}(H)\right\}},
\end{aligned}
$$

where $\rho_{n}$ is any non-zero vector in the exterior product space $\operatorname{det} P_{n} H \equiv \bigwedge^{n+1} P_{n} H$, i.e., the Grassmann product of any set of basis vectors in $\operatorname{ker} S^{* n+1}$. And when $\gamma \neq 0$

$$
\begin{aligned}
& \sigma_{S^{* n+1}, T_{\phi} S^{* n+1} R\left(T_{\phi}\right)} \\
& \quad=\frac{\operatorname{det}\left(T_{\phi} S^{* n+1} R\left(T_{\phi}\right) S^{n+1}+P\left(T_{\bar{\phi}}\right) S^{n+1}\right)}{D_{n}\left(T_{\phi}\right)} \rho_{n}^{*} \otimes T_{\phi} \rho_{n} \wedge x \otimes\left(\pi_{T_{\phi}} x\right)^{*},
\end{aligned}
$$

where $0 \neq x \in \operatorname{det} \operatorname{ker} T_{\bar{\phi}}$ and $\pi_{T_{\phi}}: H \rightarrow H / T_{\phi}(H)$ is the quotient map.

The Szegö Problem. For $\phi \in \mathcal{L}^{\infty}(\mathbb{T})$, the Toeplitz operator $T_{\phi}$ is defined on $H^{2}(\mathbb{T})$ by $T_{\phi} f=P \phi \cdot f$ where $P$ is the orthogonal projection of $\mathcal{L}^{2}(\mathbb{T})$ onto $H^{2}(\mathcal{L}(\mathbb{T}))$. Let $P_{n}$ be the orthogonal projection onto

$$
\operatorname{ker} S^{* n+1}=\bigvee\left\{1, e^{i \theta}, e^{2 i \theta}, \ldots, e^{i n \theta}\right\}=\bigvee\left\{e_{j}\right\},
$$

\footnotetext{
${ }^{1}$ For definiteness we henceforth take $R\left(T_{\phi}\right)=\left(T_{\bar{\phi}} T_{\phi}\right)^{-1} T_{\bar{\phi}}$, the Moore-Penrose inverse of $T_{\phi}$.
} 
where $S=T_{z} ;$ set $Q_{n}=1-P_{n}$ with $D_{n}\left(T_{\phi}\right) \equiv \operatorname{det}\left(P_{n} T_{\phi} P_{n}\right)$. Let $G(|\phi|) \equiv$ $\exp \frac{1}{2 \pi} \int_{0}^{2 \pi} \log \left|\phi\left(e^{i \theta}\right)\right| d \theta$.

The study of the asymptotics of $D_{n}\left(T_{\phi}\right)$ originated with the discovery in 1915 by G. Szegö that powers of the geometric mean serve as normalizing factors for the determinants $D_{n}\left(T_{\phi}\right) \equiv \operatorname{det}\left(P_{n} T_{\phi} P_{n}\right)$. Finally, in 1952 he proved the following statement:

The sharp Szegö Theorem ([30]). If $\phi\left(e^{i \theta}\right)>0$ in $[0,2 \pi]$ and $\phi^{\prime}$ satisfies a Lipschitz condition with exponent $\beta, 0<\beta \leq 1$, then

$$
\lim _{n \rightarrow \infty} \frac{D_{n}\left(T_{\phi}\right)}{[G(\phi)]^{n+1}}=\exp \left\{\frac{1}{4} \sum_{1}^{\infty} n\left|k_{n}\right|^{2}\right\},
$$

where $\sum_{1}^{\infty} k_{n} z^{n}=\frac{1}{2 \pi} \int_{0}^{2 \pi} \log \phi(\theta) \frac{1+z e^{-i \theta}}{1-z e^{-i \theta}} d \theta$, and $D_{n}\left(T_{\phi}\right)$ is the determinant of the Toeplitz matrix $\left(\left(\hat{\phi}_{i, j}\right)\right)=\left(\left(\hat{\phi}_{i-j}\right)\right), \quad 0 \leq i, j \leq n$, with $\hat{\phi}_{k}=\frac{1}{2 \pi} \int_{0}^{2 \pi} \phi(\theta) e^{-i k \theta} d \theta$.

Szegö's theorem was extended by Baxter [5], [6], Devinatz [20], Hirschman 25, Ibragimov [26], Kac [28, Widom [31, 32] and others with fewer restrictive smoothness conditions, and finally with complex symbols but originally with wind $(\phi, 0)=0$.

However, something different is required when wind $(\phi, 0) \neq 0$. For then the normalized Szegö determinant $\frac{\left|D_{n}\left(T_{\phi}\right)\right|}{G(|\phi|)^{n+1}} \rightarrow 0$ for smooth $\phi$. Thus, to solve the problem of finding a full analog of the Szegö theorem when wind $(\phi, 0) \neq 0$ it is necessary to

a) produce a normalization divisor $A_{n}$ so that the sequence $\frac{D_{n}\left(T_{\phi}\right)}{A_{n}}$ has a natural limit,

b) explain the limit in terms of topological and analytic data implicit in the symbol.

The main results below are contained in Theorems 1.1, 1.2, 1.3, and 1.6.

If $\phi=\psi z^{\gamma}$, wind $(\psi, 0)=0$, put

$$
\bar{g} \equiv \exp \left(\sum_{n=1}^{\infty}(\log \psi)_{-n} t^{-n}\right), \quad f \equiv \exp \left(\sum_{n=0}^{\infty}(\log \psi)_{n} t^{n}\right)
$$

so that $\psi=f \bar{g}$. Let $H_{\omega}$ be the Hankel operator with symbol $\omega$. Then, with $E(\psi) \equiv$ $\exp \sum_{k=1}^{\infty} k(\log \psi)_{k}(\log \psi)_{-k}$, and $\mathbf{G}(\phi) \equiv \operatorname{det}_{*} \partial\left\{T_{\psi z \gamma}+\mathcal{L}^{1}(H), S+\mathcal{L}^{1}(H)\right\}$, a Steinberg symbol determinant defined in terms of the cosets relative to the trace ideal of the Toeplitz operator and the shift:

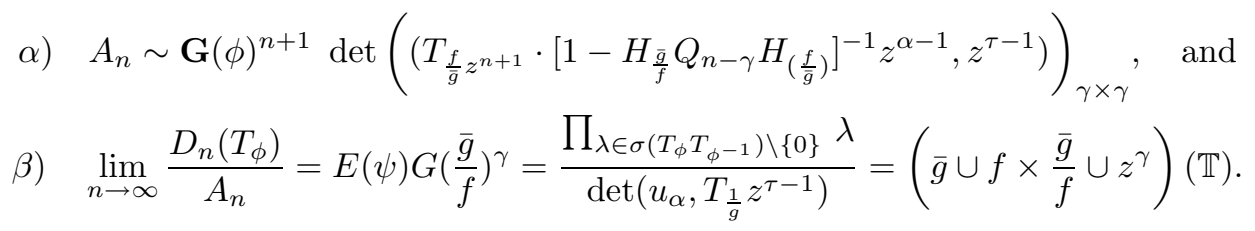

When $\phi=\psi z^{\gamma}$ and wind $(\psi, 0)=0$, we will prove that

$$
\mathbf{G}(\phi)^{n+1}=\operatorname{det}_{*} \partial\left\{T_{\psi z^{\gamma}}+\mathcal{L}^{1}(H), S^{n+1}+\mathcal{L}^{1}(H)\right\}=(-1)^{(n+1) \gamma} G(\psi)^{n+1} .
$$

Although it is known (see $\S 1.0 .1$ and $\S 5$ ) that

$$
\lim _{n \rightarrow \infty} \frac{D_{n}\left(T_{\phi}\right)}{(-1)^{(n+1) \gamma} G(\psi)^{n+1} \operatorname{det}\left(T_{\frac{f}{g} z^{n+1}} z^{\alpha-1}, z^{\tau-1}\right)}=E(\psi) G\left(\frac{\bar{g}}{f}\right)^{\gamma}
$$


for many symbols, the limit on the left does not exist in general. There are smooth symbols $\phi$ so that $D_{n}\left(T_{\phi}\right) \neq 0$, yet $D_{\gamma-1}\left(T_{\frac{f}{g}} z^{n+1}\right)=\operatorname{det}\left(T_{\frac{f}{q}} z^{n+1} z^{\alpha-1}, z^{\tau-1}\right)=0$ for infinitely many $n$. See $\S 6$ below. Thus the corrected nomalization provided by $\alpha$ ) is necessary to find the leading term in the asymptotics of $D_{n}\left(T_{\phi}\right)$ and thereby get the general limit theorem in $\beta$ ).

The topological formula $\left(\bar{g} \cup f \times \frac{\bar{g}}{f} \cup z^{\gamma}\right)(\mathbb{T})$ denotes the holonomy of a flat bundle taken over the unit circle $\mathbb{T}$. Both the bundle and its connection are defined by the factorization $\phi=z^{\gamma} f \bar{g}$. This will be discussed in $\S 1.2$.

Theorem 1.1. Suppose $\phi \in \mathcal{L}^{\infty}(\mathbb{T})$ where $T_{\phi}$ is Fredholm, injective, $T_{\phi} T_{\phi^{-1}}-$ $1 \in \mathcal{L}^{1}\left(H^{2}(\mathbb{T})\right)$. Let $\left\{w_{\alpha}\right\}_{1}^{\gamma}$ be any basis for $\operatorname{ker} T_{\phi^{-1}}$ and let $\left\{u_{\alpha}\right\}_{1}^{\gamma}$ be any set of maximal root vectors relative to $\left\{(-1)^{m_{\alpha}} w_{\alpha}\right\}_{1}^{\gamma}$ and $T_{\phi} T_{\phi^{-1}}$, i.e., $\left(T_{\phi} T_{\phi^{-1}}\right)^{m_{\alpha}} u_{\alpha}=$ $(-1)^{m_{\alpha}} w_{\alpha}$ where $m_{\alpha}=\max \left\{m \in Z_{+}: \exists x\right.$ so that $\left.\left(T_{\phi} T_{\phi^{-1}}\right)^{m} x=w_{\alpha}\right\}$. Then, if $\left\{w_{\alpha}^{(n)}\right\}_{1}^{\gamma}$ is any sequence of bases in $\operatorname{ker} S^{* n+1} R\left(T_{\phi}\right) S^{n+1}$ for which $w_{\alpha}^{(n)}$ converges to $w_{\alpha}$ for $1 \leq \alpha \leq \gamma$ and if $\left\{t_{\beta}\right\}_{1}^{\gamma}$ is any basis of $\operatorname{ker} T_{\bar{\phi}}$, then for $n \gg 0$,

i)

$$
D_{n}\left(T_{\phi}\right)=\mathbf{G}(\phi)^{n+1} \cdot \operatorname{det}\left(w_{\alpha}^{(n)}, S^{* n+1} t_{\tau}\right)_{\gamma \times \gamma^{\cdot}}\left[\frac{\prod_{\lambda \in \sigma\left(T_{\phi} T_{\phi^{-1}}\right) \backslash\{0\}} \lambda}{\operatorname{det}\left(u_{\alpha}, t_{\tau}\right)}\right][1+o(1)] .
$$

If in addition, $\phi \in \operatorname{Lip}_{\beta}, \frac{1}{2}<\beta<1$, then for $n \gg 0$,

$$
\begin{aligned}
D_{n}\left(T_{\phi}\right)= & \mathbf{G}(\phi)^{n+1} \cdot \operatorname{det}\left(w_{\alpha}^{(n)}, S^{* n+1} t_{\tau}\right)_{\gamma \times \gamma} \\
& \cdot\left[\frac{\prod_{\lambda \in \sigma\left(T_{\phi} T_{\phi}-1\right) \backslash\{0\}}^{\lambda}}{\operatorname{det}\left(u_{\alpha}, t_{\tau}\right)}\right]\left[1+O\left(n^{(1-2 \beta)}\right)\right] .
\end{aligned}
$$

Theorem 1.2. Let $\phi=\psi z^{\gamma}$ for integer-valued $\gamma>0$ with normalized WienerHopf factorization $\psi=f \bar{g}$ and let $f$ and $g$ be outer functions in $K_{2,2}^{\frac{1}{2}, \frac{1}{2}}=\{\phi$ : $\left.\phi \in L^{\infty}, \sum_{-\infty}^{\infty}|\hat{\phi}(k)|^{2}|k|<\infty\right\}$. Let $\left\{e_{\alpha}\right\}_{1}^{\gamma} \equiv\left\{1, z, \ldots, z^{\gamma-1}\right\}$ denote the standard basis for $\operatorname{ker} T_{\bar{z} \gamma}$, and let $u_{1}, u_{2}, \ldots, u_{\gamma}$ be associated root vectors of the nilpotent part of the operator $T_{\phi} T_{\phi^{-1}}$ so that $u_{\alpha}$ has maximal order with respect to $(-1)^{m_{\alpha}} T_{f} e_{\alpha}$, , for $\alpha=1,2, \ldots, \gamma$, i.e., $\left(T_{\phi} T_{\phi^{-1}}\right)^{m_{\alpha}} u_{\alpha}=(-1)^{m_{\alpha}} T_{f} e_{\alpha}$ where $m_{\alpha}=$ $\max \left\{m: \exists x\right.$ so that $\left.\left(T_{\phi} T_{\phi^{-1}}\right)^{m} x=T_{f} e_{\alpha}\right\}$. Then with $E(\psi)=\operatorname{det}\left(T_{\psi} T_{\psi^{-1}}\right)=$ $\exp \sum_{k=1}^{\infty} k(\log f)_{k}(\log \bar{g})_{-k}$, we have

a) $\sum_{1}^{\gamma} m_{\alpha}=\operatorname{dim} \operatorname{root}$ space $\left[Q_{\gamma-1}\left(I-H_{\psi} H_{\frac{1}{\psi} \mid \operatorname{ran}\left(Q_{\gamma-1)}\right)}\right)\right.$,

b) $E(\psi) G\left(\frac{\bar{g}}{f}\right)^{\gamma}=\frac{\prod_{\lambda \in \sigma\left(T_{\phi} T_{\phi}-1\right) \backslash\{0\}} \lambda}{\operatorname{det}\left(u_{\alpha}, T_{\frac{1}{g}} e_{\tau}\right)}$,

c) when $m_{\alpha}=0$ for $\alpha=1, \ldots, \gamma$, then $E(\psi) G\left(\frac{\bar{g}}{f}\right)^{\gamma}=\frac{\prod_{\lambda \in \sigma\left(T_{\phi} T_{\phi-1}\right) \backslash\{0\}} \lambda}{D_{\gamma-1}\left(T_{\frac{f}{g}}\right)}$.

Note that $m_{\alpha}=0$ if $\phi$ is unimodular.

Theorem 1.3. Suppose $\phi=\psi z^{\gamma}$ where $\gamma \in Z_{+}$and $\psi \in \operatorname{Lip}_{\beta}, \frac{1}{2}<\beta<1$ and $T_{\psi}$ is invertible. Then we have $\psi=f \bar{g}$ where $f, g$ and $\frac{1}{f}, \frac{1}{g} \in \operatorname{Lip}_{\beta} \cap H^{\infty}(\mathbb{T}), \frac{1}{2}<\beta<1$, 


$$
\begin{aligned}
& \text { if } e_{\alpha}=z^{\alpha}, \alpha=1,2, \ldots, \gamma, \text { and } b=\frac{\bar{g}}{f}, \\
& \begin{aligned}
D_{n}\left(T_{\phi}\right)=\mathbf{G}(\phi)^{n+1} E(\psi) G(b)^{\gamma} \\
\cdot \operatorname{det}\left(\left(T_{\frac{f}{g} z^{n+1}} \cdot\left[1-H_{\frac{\bar{g}}{f}} Q_{n-\gamma} H_{\left(\frac{f}{g}\right)}\right]^{-1} e_{\alpha}, e_{\tau}\right)\right)_{\gamma \times \gamma}\left[1+O\left(n^{1-2 \beta}\right)\right] . \\
\text { If } \phi \in K_{2,2}^{\frac{1}{2}, \frac{1}{2}}, \text { then } \\
D_{n}\left(T_{\phi}\right)=\mathbf{G}(\phi)^{n+1} E(\psi) G(b)^{\gamma} \\
\cdot \operatorname{det}\left(\left(T_{\frac{f}{g}} z^{n+1} \cdot\left[1-H_{\frac{\bar{g}}{f}} Q_{n-\gamma} H_{\left(\frac{f}{g}\right)}\right]^{-1} e_{\alpha}, e_{\tau}\right)\right)_{\gamma \times \gamma}[1+o(1)] .
\end{aligned}
\end{aligned}
$$

The proof uses Theorem 1.1 and the identity

$$
\begin{aligned}
\operatorname{det} & \left(\left(S^{n+1}\left[S^{* n+1-\gamma} T_{\psi}^{-1} S^{n+1-\gamma}\right]^{-1} e_{\alpha}, T_{\frac{1}{g}} e_{\tau}\right)\right) \\
& =\operatorname{det}\left(\left(T_{\frac{f}{g} z^{n+1}} \cdot\left[1-H_{\frac{\bar{g}}{f}} Q_{n-\gamma} H_{\left(\frac{f}{g}\right)}\right]^{-1} T_{\bar{g}} e_{\alpha}, e_{\tau}\right)\right)_{\gamma \times \gamma} .
\end{aligned}
$$

1.0.1. Relation to other results. Undefined factors in the BöttcherSilbermann approximation. An important step towards the non-zero index case, was made when M. Fisher and E. Hartwig [21] used Jacobi's theorem on the conjugate minors of the adjugate matrix formed from the cofactors of $D_{n}\left(T_{\phi}\right)$ to give asymptotic information even when $\phi$ is piecewise continuous and wind $(\phi, 0) \neq 0$.

The pioneering Fisher-Hartwig results were refined by Böttcher and Silbermann [10] where it was assumed that $\phi=a z^{\gamma}$, with $a \in \operatorname{Lip}_{\beta}, \beta>\frac{1}{2}$, with the WienerHopf factorization $a=a_{+} a_{-}$, and invertible $T_{a}$. For integer $\gamma \geq 0$ and $n \gg 0$, the Böttcher-Silbermann approximation is:

$$
\begin{aligned}
D_{n}\left(T_{a z^{\gamma}}\right)= & (-1)^{(n+1) \gamma} G(a)^{n+1} E(a) G(b)^{\gamma} \\
& \cdot\left[\operatorname{det}\left(\begin{array}{ccc}
\hat{c}_{-n-1} & \cdots & \hat{c}_{-n+\gamma-2} \\
\vdots & \vdots & \vdots \\
\hat{c}_{-n-\gamma} & \cdots & \hat{c}_{-n-1}
\end{array}\right)+O\left(n^{-3 \beta}\right)\right]\left[1+O\left(n^{1-2 \beta)}\right)\right],
\end{aligned}
$$

with $c=\frac{a_{+}}{a_{-}}, b=\frac{a_{-}}{a_{+}}$, and $\hat{c}_{j}$ denoting the $j$ th Fourier coefficient, and $E(a)=$ $\exp \sum_{k=1}^{\infty} k\left(\log a_{+}\right)_{k}\left(\log a_{-}\right)_{-k}$. As noted above, this approximation fails to give a limit theorem. In $\S 6$ an example is given of a rational symbol for which the factor $\operatorname{det}\left(C_{n, \gamma}\right)=0$ for infinitely many $n$ and yet $D_{n}(\phi) \neq 0$ for $n \gg 0$. In this case, for a subsequence $\left\{n_{j}\right\}$ for which $\operatorname{det}\left(C_{n_{j}, \gamma}\right)=0$, the Fisher-Hartwig, Böttcher-Silbermann assertion becomes the statement that there is an unspecified denominator, $\left[O\left(n_{j}^{-3 \beta}\right)\right]$, so that

$$
\frac{D_{n_{j}}\left(T_{a z^{\gamma}}\right)}{(-1)^{\left(n_{j}+\gamma\right) \gamma} G(a)^{n_{j}+1}\left[O\left(n_{j}^{-3 \beta}\right)\right]}=E(a) G(b)^{\gamma}\left[1+O\left(n_{j}^{1-2 \beta)}\right]\right), \quad j=1, \ldots, \infty .
$$

Note that the geometric series for

$$
\left[1-H_{\frac{\bar{g}}{f}} Q_{n-\gamma} H_{\left(\frac{f}{\bar{g}}\right)}\right]^{-1}=1+\sum_{k=1}^{\infty}\left(H_{\frac{\bar{g}}{f}} Q_{n-\gamma} H_{\left(\frac{f}{\bar{g}}\right)}\right)^{k}
$$

of Theorem 1.3, which is valid for large $n$, provides a correction for $\operatorname{det} C_{n, \gamma}$ since we have the following remark. 
Remark 1.2. If $a=a_{+} a_{-} \in L^{\infty}(\mathbb{T})$ with $a_{+}$and $\bar{a}_{-}$outer in $H^{2}(\mathbb{T})$ and with $T_{a}$ invertible, then

$$
\begin{gathered}
\operatorname{ker} T_{\bar{a} \bar{z}^{\gamma}}=\bigvee\left\{\frac{1}{\bar{a}_{-}}, \frac{z}{\bar{a}_{-}}, \ldots, \frac{z^{\gamma-1}}{\bar{a}_{-}}\right\}, \\
\operatorname{ker} T_{\left(a z^{\gamma}\right)^{-1}}=\bigvee\left\{a_{+}, z a_{+}, z^{2} a_{+}, \ldots, z^{\gamma-1} a_{+}\right\},
\end{gathered}
$$

and if we set $w_{\alpha}=z^{\alpha-1} a_{+}$and $y_{\tau}=\frac{z^{\tau-1}}{\bar{a}_{-}}$for $\alpha, \tau=1, \ldots, \gamma$, then

$$
\begin{aligned}
\operatorname{det}\left(C_{n, \gamma}\right) & =\operatorname{det}\left(w_{\alpha}, S^{* n+1} y_{\tau}\right) \\
& =\operatorname{det}\left(\begin{array}{ccc}
\hat{c}_{-n-1} & \cdots & \hat{c}_{-n+\gamma-2} \\
\vdots & \vdots & \vdots \\
\hat{c}_{-n-\gamma} & \cdots & \hat{c}_{-n-1}
\end{array}\right)=\operatorname{det}\left(T_{\frac{a_{+}}{a_{-}} z^{n+1}} z^{\alpha}, z^{\tau}\right) .
\end{aligned}
$$

Identification of factors in the Widom approximation. Harold Widom 32 treats symbols having the form $\phi=f \bar{g} z^{\gamma}$ where $f$ is piecewise $C^{\infty}$ but $g$ is not in $C^{\infty}$, i.e., there are finitely many points at which certain higher order derivatives are piecewise continuous. The aim of his work is to produce an asymptotic formula for $D_{n}\left(T_{\phi}\right)$ in the presence of such higher order singularities and non-vanishing index. His main result is

$$
D_{n}\left(T_{\phi}\right)=G(f \bar{g})^{n+1} n^{-e}\left(c_{n}+o(1)\right),
$$

where $e$ is a non-negative exponent depending on the nature of the singularities of $\phi$ (these are points with no neighborhoods in which $\phi \in C^{\infty}$ ) and $c_{n}$ is a bounded sequence given by "a rather complicated but (in principle) perfectly explicit formula."

Comparison of the Widom result and Theorem 1.1 gives

$$
n^{-e}\left[c_{n}+o(1)\right]=(-1)^{(n+1) \gamma} \operatorname{det}\left(S^{n+1} w_{\alpha}^{(n)}, t_{\tau}\right) \frac{\prod_{\lambda \in \sigma\left(T_{\phi} T_{\phi}-1\right) \backslash\{0\}} \lambda}{\operatorname{det}\left(u_{\alpha}, t_{\tau}\right)} .
$$

For a certain class of generating functions $\phi$ Widom succeeds in showing that for almost all $x$ not in the range of $\phi$, the sequence $c_{n}=c_{n}(x)$ corresponding to the symbol $\phi-x$ remains bounded away from zero and he says that "we have no doubt that this is always true" - but "we have not been able to prove that this is always the case".

Note that for fixed $x$ the sequence $c_{n}(x)$ may approach zero very rapidly. For example, suppose that $f \in \operatorname{Lip}(\beta)$ with $\beta>\frac{1}{2}$ and is piecewise in $C^{\infty}$, while $g \in C^{\infty}$. Since $\operatorname{ker} T_{\bar{\phi}}=T_{\frac{1}{q}}\left\{1, z, z^{2}, \ldots, z^{\gamma-1}\right\}$, it follows that $\left\|S^{* n+1} y_{\tau}\right\|=o\left(n^{-p}\right), \forall p>0$, and therefore $c_{n}+o(1)=o\left(n^{-p}\right)$ for any $p>0$.

On the other hand, if $c_{n}$ is bounded away from zero, Theorem 1.3 says

$$
c_{n} \sim(-1)^{(n+1) \gamma} n^{e} E(f \bar{g}) G\left(\frac{\bar{g}}{f}\right)^{\gamma} \cdot \operatorname{det}\left(\left(T_{\frac{f}{g} z^{n+1}} \cdot\left[1-H_{\frac{\bar{g}}{f}} Q_{n-\gamma} H_{\left(\frac{f}{g}\right)}\right]^{-1} e_{\alpha}, e_{\tau}\right)\right)_{\gamma \times \gamma} .
$$

Some key relations. The vectors $\sigma_{S^{* n+1}, T_{\phi} S^{* n+1} T_{\phi}{ }^{-1}}$ and $\sigma_{1, T_{\phi} S^{* n+1} R\left(T_{\phi}\right) S^{n+1}}$ were calculated in [15]. The following is one consequence of these calculations that is fundamental for the present investigation. 
The appearance of root vectors. The limit in Theorem 1.1 above is obtained from the convergence of the sequence of perturbation vectors

$$
\begin{aligned}
\lim _{n \rightarrow \infty} & \sigma_{1, T_{\phi} S^{* n+1} R\left(T_{\phi}\right) S^{n+1}} \\
& =\sigma_{1, T_{\phi} T_{\phi^{-1}}}=\frac{\prod_{\lambda \in \sigma\left(T_{\phi} T_{\phi^{-1}}\right) \backslash\{0\}} \lambda}{\operatorname{det}\left(u_{\alpha}, y_{\beta}\right)}\left(\bigwedge_{k=1}^{\gamma} w_{k}\right) \otimes\left(\bigwedge_{k=1}^{\gamma}\left[y_{k}+\operatorname{ran} T_{\phi}\right]\right)^{*} .
\end{aligned}
$$

This form for the right-hand side of the limiting perturbation vector $\sigma_{1, T_{\phi} T_{\phi}-1}$ follows from an apparently new observation about finite matrices given in Proposition 2.1 below:

If $X$ is an operator on Hilbert space so that $X=$ identity + trace class, and $\left\{w_{\alpha}\right\}_{1}^{\gamma}$ is any basis for $\operatorname{ker} X,\left\{y_{\alpha}\right\}_{1}^{\gamma}$ is any orthonormal basis for $\operatorname{ker} X^{*}$, and if $\left\{u_{\alpha}\right\}_{1}^{\gamma}$ is a corresponding set of root vectors of maximal height for $\left\{w_{\alpha}\right\}_{1}^{\gamma}$, i.e., $(X)^{\max } u_{\alpha}=$ $w_{\alpha}, \alpha=1,2, \ldots, \gamma$. Then with $m_{\alpha}+1$ denoting the algebraic multiplicty of $w_{\alpha}$,

$$
(-1)^{\sum_{\alpha=1}^{\gamma} m_{\alpha}} \cdot \prod_{\lambda \in \sigma(X) \backslash\{0\}} \lambda \cdot \frac{\left\|\bigwedge w_{j}\right\|^{2}}{\operatorname{det}\left(u_{j}, y_{i}\right)}=\operatorname{det}\left(X+\sum_{j=1}^{\gamma} y_{j} \otimes w_{j}\right) .
$$

Three examples. Consider the matrix given by $A=\left(\begin{array}{llll}1 & 2 & 1 & 0 \\ 0 & 1 & 3 & 0 \\ 0 & 0 & 1 & 0 \\ 1 & 0 & 0 & 0\end{array}\right)$ with minimal polynomial $x(x-1)^{3}$. Then an orthonormal basis for $\operatorname{ker} A$ is $w_{1}=(0,0,0,1)$ and an orthonormal basis for $\operatorname{ker} A^{*}$ is $y_{1}=\left(\frac{1}{\sqrt{3} 1},-\frac{2}{\sqrt{3} 1}, \frac{5}{\sqrt{3} 1},-\frac{1}{\sqrt{3} 1}\right)$. Since $m_{\alpha}=0$, $u_{1}=w_{1}$ is a root vector of maximal height lying over the kernel vector of $A$, i.e., $(A)^{0} u_{1}=w_{1}$.

Then, $\operatorname{det}\left(w_{1}, y_{1}\right)=-\frac{1}{\sqrt{3} 1}$ so that

$$
\frac{1}{\operatorname{det}\left(u_{1}, y_{1}\right)}=-\sqrt{31}=\operatorname{det}\left(A+y_{1} \otimes w_{1}\right)=\operatorname{det}\left(\begin{array}{cccc}
1 & 2 & 1 & \frac{1}{\sqrt{3} 1} \\
0 & 1 & 3 & -\frac{2}{\sqrt{31}} \\
0 & 0 & 1 & \frac{5}{\sqrt{31}} \\
1 & 0 & 0 & -\frac{1}{\sqrt{3} 1}
\end{array}\right) .
$$

For another example, take $B=\left(\begin{array}{cccc}0 & 2 & 1 & 0 \\ 0 & 0 & 3 & 0 \\ 0 & 0 & 0 & 0 \\ 1 & 0 & 0 & -1\end{array}\right)$. The minimal polynomial is $x^{3}(x+1)$, an orthonormal basis for $\operatorname{ker} B$ is $w_{1}=\left(\frac{1}{\sqrt{2}}, 0,0, \frac{1}{\sqrt{2}}\right)$ while $y_{1}=(0,0,1,0)$ is an orthonormal basis for $\operatorname{ker} X^{*} \cdot u_{1}=\left(0,0, \frac{\sqrt{2}}{12}, 0\right)$ is a root vector of maximal height lying over $w_{1}$, i.e., $B^{2} u_{1}=w_{1}$. The length $m_{\alpha}+1$ of the corresponding Jordan chain is 3 , but $\prod_{\lambda \in \sigma(X) \backslash\{0\}} \lambda=-1$. Hence we have

$$
y_{1} \otimes w_{1}=\left(\begin{array}{cccc}
0 & 0 & 0 & 0 \\
0 & 0 & 0 & 0 \\
(1 / 2) \cdot \sqrt{2} & 0 & 0 & (1 / 2) \cdot \sqrt{2} \\
0 & 0 & 0 & 0
\end{array}\right)
$$


and

$$
\begin{aligned}
\operatorname{det}\left(B+y_{1} \otimes w_{1}\right) & =\operatorname{det}\left(\begin{array}{cccc}
0 & 2 & 1 & 0 \\
0 & 0 & 3 & 0 \\
(1 / 2) \cdot \sqrt{2} & 0 & 0 & (1 / 2) \cdot \sqrt{2} \\
1 & 0 & 0 & -1
\end{array}\right) \\
& =-6 \sqrt{2}=\frac{(-1)^{3}}{\operatorname{det}\left(u_{1}, y_{1}\right)}=\frac{(-1)^{3}}{\frac{\sqrt{2}}{12}} .
\end{aligned}
$$

For a Hilbert space example, let us consider $B \equiv 1-a \otimes b$ where $(a, b)=1$. Then ker $B$ has basis $\{a\}$ and $a$ is a maximal root vector lying over itself. Hence, since the non-zero eigenvalues of $B$ are all equal to 1 , we get $\sigma_{1, B}=a \otimes(a+\operatorname{ran} B)^{*}$. Thus, $\|a+\operatorname{ran} B\|=\left\|P\left(B^{*}\right) a\right\|$. Now $P\left(B^{*}\right)=\frac{b}{\|b\|} \otimes \frac{b}{\|b\|}$, and $(a, b)=1$, so we have $\left\|P\left(B^{*}\right) a\right\|=\frac{1}{\|b\|}$. Consequently, $\left\|\sigma_{1, B}\right\|=\|a\| \cdot\|b\|$.

Now let $\phi=z-\lambda,\|\lambda\|<1$. Then $T_{\phi} T_{\phi^{-1}}=1-e_{0} \otimes e_{\bar{\lambda}}$, where $e_{\bar{\lambda}}=\sum_{k=0}^{\infty} \bar{\lambda}^{k} z^{k}$. But $\left\|e_{0}\right\|=1$ and $\left\|e_{\bar{\lambda}}\right\|=\frac{1}{\sqrt{1-|\lambda|^{2}}}$. Thus, by the paragraph above, $\left\|\sigma_{1, T_{\phi} T_{\phi^{-}}}\right\|=$

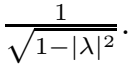

The invariant vector. Suppose that for a Fredholm operator $B$ we have $1-B \in$ $\mathcal{L}^{1}$. We observe now that if we pick any basis $\left\{(-1)^{m_{\alpha}} w_{\alpha}\right\}$ for $\operatorname{ker} B$, and any corresponding basis of top root vectors $\left\{u_{\alpha}\right\}$ for Jordan chains lying over the kernel vectors, we may form the vector

$$
\mathcal{I}(B) \equiv\left(\bigwedge w_{\alpha}\right)^{*} \otimes\left(\bigwedge u_{\alpha}+H / B H\right) .
$$

a) $\mathcal{I}(B)$ is invariant under the choice of the root vectors $\left\{u_{\alpha}\right\}$ for a fixed choice of $\left\{w_{\alpha}\right\}$.

b) $\mathcal{I}(B)$ is invariant under the choice of null vectors $\left\{w_{\alpha}\right\}$.

Pairing the invariant vector with the perturbation vector gives $\prod_{\lambda \in \sigma(B) \backslash\{0\}} \lambda$ so that

$$
\sigma_{1, B}=\prod_{\lambda \in \sigma(B) \backslash\{0\}} \lambda \cdot \mathcal{I}(B) .
$$

But Theorem 1.1 exploits the fact that we have the freedom to choose a different basis $\left\{t_{\tau}\right\}$ for the cokernel space. We obtain then the same vector $\sigma_{1, B}$, but another numerical coefficient multiplying the corresponding tensor product. In the preceding theorems and Theorem 1.6 below we exploit this freedom to choose bases for the cokernel space corresponding to the factorization $\phi=\psi z^{\gamma}$, and in Theorem A we obtain an additional meaning for the resulting coefficient in terms of holonomy.

The separation property for finite matrices or for operators of the form $1+$ trace class. Suppose that $A$ and $B$ are stationary 2 Fredholm operators. Then we have the Riesz reduction $H^{2}(\mathbb{T})=R_{\infty}(A)+N_{\infty}(A)=R_{\infty}(B)+N_{\infty}(B)$, where now we use the notation $N_{\infty}(T)=\bigcup_{n=1}^{\infty} \operatorname{ker} T^{n}$ and $R_{\infty}(T)=\bigcap_{n=1}^{\infty} \operatorname{ran} T^{n}$.

\footnotetext{
${ }^{2}$ Recall that $T$ is stationary if there exist a finite $p$ so that $N_{p} \equiv \operatorname{ker} T^{p}=\operatorname{ker} T^{p+1}=\cdots=$ $N_{\infty}(T)$ and $R_{p}(T) \equiv \operatorname{ran} T^{p}=R_{p+1}(T)=\cdots=R_{\infty}(T)$.
} 
Select a basis $\bigwedge_{1}^{\gamma}(-1)^{m_{j}} w_{j}^{A}$ for $\operatorname{det}$ ker $A$ with associated maximal root vectors $\left\{u_{j}^{A}\right\}_{j=1}^{\gamma}$ and form the invariant vector

$$
\mathcal{I}(A)=\left(\bigwedge_{1}^{\gamma} w_{j}^{A}\right)^{*} \otimes\left[\bigwedge_{1}^{\gamma} u_{j}^{A}+\operatorname{ran} A\right]
$$

Similarly, select a basis $\bigwedge_{1}^{\gamma}(-1)^{m_{\tau}} w_{\tau}^{B}$ for det ker $B$ and associated maximal root vectors $\left\{u_{\tau}^{B}\right\}_{\tau=1}^{\mu}$ lying over these basis vectors and form the invariant vector

$$
\mathcal{I}(B)=\left(\bigwedge_{1}^{\mu} w_{j}^{B}\right)^{*} \otimes\left[\bigwedge_{1}^{\mu} u_{j}^{B}+\operatorname{ran} B\right]
$$

Let $M$ be the linear map on $H$ so that $M=0$ on $R_{\infty}(A)$ and $M$ maps $w_{j}^{A}$ to $u_{j}^{A}$ for all $j=1, \ldots, \gamma$.

Let $L$ be the linear map on $H$ so that $L=0$ on $R_{\infty}(B)$ and $L$ maps $w_{\tau}^{B}$ to $u_{\tau}^{B}$ for all $\tau=1, \ldots, \mu$. Then

$$
\sigma_{A, B}=\operatorname{det}\left[(A+M)^{-1}(B+L)\right] \cdot \mathcal{I}(A) \otimes \mathcal{I}(B)^{*} .
$$

Factorization. Suppose $A$ and $B$ are finite matrices (alternately, $A$ and $B$ are of the form $\left.1+\mathcal{L}^{1}\right)$. The indices are both zero and the given description of the vector $\sigma_{A, B}$ applies. The coefficient factors as $\frac{\operatorname{det}(B+L)}{\operatorname{det}(A+M)}=\frac{\prod_{\lambda_{B} \in \sigma(B) \backslash\{0\}} \lambda_{B}}{\prod_{\lambda_{A} \in \sigma(A) \backslash\{0\}} \lambda_{A}}$, and therefore

$$
\sigma_{A, B}=\sigma_{1, A}^{*} \otimes \sigma_{1, B}=\sigma_{A, 1} \otimes \sigma_{1, B}
$$

\subsection{Perturbation vectors and the Szegö limit theorem.}

Lemma 1.1. Suppose $T_{\phi}$ is injective and $T_{\phi} T_{\phi^{-1}}-1 \in \mathcal{L}^{1}(\mathbb{T})$. Let $w_{1}, \ldots, w_{\gamma}$ be any basis for $\operatorname{ker} T_{\phi^{-1}}$. Then there is a basis $\left\{w_{k}^{(n)}\right\}$ in $\operatorname{ker}\left[S^{* n+1} R\left(T_{\phi}\right) S^{n+1}\right]$ for $n \gg 0$ so that $w_{k}^{(n)}$ converges to $w_{k}$ for $1 \leq k \leq \gamma$.

Lemma 1.2. Suppose $D_{n}\left(T_{\phi}\right) \neq 0$, and $\left\{t_{\tau}\right\}_{1}^{\gamma}$ is a basis for $\operatorname{ker} T_{\bar{\phi}}$, while $\left\{w_{k}^{(n)}\right\}_{k=1}^{\gamma}$ is any basis for $\operatorname{ker}\left[S^{* n+1} R\left(T_{\phi}\right) S^{n+1}\right]$. Then

$$
\begin{aligned}
\sigma_{1, T_{\phi} S^{* n+1} R\left(T_{\phi}\right) S^{n+1}=} & \frac{D_{n}\left(T_{\phi}\right)}{\mathbf{G}(\phi)^{n+1} \cdot \operatorname{det}\left(\left(w_{\alpha}^{(n)}, S^{* n+1} t_{\tau}\right)\right)} \\
& \cdot\left(\bigwedge_{k=1}^{\gamma} w_{k}^{(n)}\right) \otimes\left(\bigwedge_{k=1}^{\gamma}\left[t_{k}+\operatorname{ran} T_{\phi}\right]\right)^{*},
\end{aligned}
$$

and if top root vectors $u_{\alpha}$ of $T_{\phi} T_{\phi^{-1}}$ are chosen to lie over the kernel vectors $(-1)^{m_{\alpha}} w_{\alpha} \in \operatorname{ker} T_{\phi^{-1}}$, i.e., $\left(T_{\phi} T_{\phi^{-1}}\right)^{m_{\alpha}} u_{\alpha}=(-1)^{m_{\alpha}} w_{\alpha}$ where $m_{\alpha}=\max \{m \in$ $Z_{+}: \exists x$ so that $\left(T_{\phi} T_{\phi^{-1}}\right)^{m} x=w_{\alpha}$, we have

$$
\sigma_{1, T_{\phi} T_{\phi}-1}=\frac{\prod_{\lambda \in \sigma}\left(T_{\phi} T_{\phi^{-1}}\right) \backslash\{0\} \lambda}{\operatorname{det}\left(u_{\alpha}, t_{\tau}\right)}\left(\bigwedge_{k=1}^{\gamma} w_{k}\right) \otimes\left(\bigwedge_{k=1}^{\gamma}\left[t_{k}+\operatorname{ran} T_{\phi}\right]\right)^{*} .
$$

If $w_{k}^{(n)} \rightarrow w_{k}$ it is clear that this representation of $\sigma_{1, T_{\phi} T_{\phi^{-1}}}$ immediately gives a limit theorem for the coefficients of the two perturbation vectors

$$
\lim _{n \rightarrow \infty} \frac{D_{n}\left(T_{\phi}\right)}{\mathbf{G}(\phi)^{n+1} \cdot \operatorname{det}\left(w_{\alpha}^{(n)}, S^{* n+1} t_{\tau}\right)}=\frac{\left.\prod_{\lambda \in \sigma\left(T_{\phi} T_{\phi}-1\right.}\right) \backslash\{0\}}{\operatorname{det}\left(u_{\alpha}, t_{\tau}\right)} .
$$


The factor $\mathbf{G}(\phi)^{n+1}$ is explictly evaluated using Theorem 1.5 below. If $\phi=\psi z^{\gamma}$, then $\mathbf{G}(\phi)^{n+1}=(-1)^{\gamma(n+1)} \cdot G(\psi)^{n+1}=\operatorname{det}_{*} \partial\left\{T_{\phi}+\mathcal{L}^{1}(H), T_{z^{n+1}}+\mathcal{L}^{1}(H)\right\}=$ $\phi \cup z^{n+1}(\Sigma)$, the holonomy of a bundle $\phi \cup z^{n+1}$ over the unit circle $\mathbb{T}$ which is computed by an integral.

The form of the perturbation vector in Lemma 1.2. Using Proposition 1.1 it was shown in [15], equation (73), that when $T_{\phi}$ is injective, Fredholm, $\operatorname{det}\left(P_{n} T_{\phi} P_{n}\right) \neq 0$, and $\left\{v_{\alpha}^{(n)}\right\}_{1}^{\gamma}$ is a basis for $\operatorname{ker} S^{* n+1} R\left(T_{\phi}\right) S^{n+1}$, then

$$
\begin{aligned}
\sigma_{1, T_{\phi} S^{* n+1} R\left(T_{\phi}\right) S^{n+1}=} & \frac{D_{n}\left(T_{\phi}\right)}{\operatorname{det}_{*} \partial\left\{T_{\phi}+\mathcal{L}^{1}(H), S^{n+1}+\mathcal{L}^{1}(H)\right\}} \\
& \cdot\left(\bigwedge_{\alpha=1}^{\gamma} v_{\alpha}^{(n)}\right) \otimes\left(\bigwedge_{\alpha=1}^{\gamma}\left[S^{n+1} v_{\alpha}^{(n)}+\operatorname{ran} T_{\phi}\right]\right)^{*} .
\end{aligned}
$$

Since the cosets $S^{n+1} v_{\alpha}^{(n)}+\operatorname{ran} T_{\phi}=\sum_{1}^{\gamma}\left(S^{n+1} v_{\alpha}^{(n)} y_{\beta}\right) y_{\beta}+\operatorname{ran} T_{\phi}$, for any orthonormal basis $\left\{y_{\alpha}\right\}_{1}^{\gamma} \subset \operatorname{ker} T_{\bar{\phi}}$, it follows that $\left(\bigwedge_{\alpha=1}^{\gamma}\left[S^{n+1} v_{\alpha}^{(n)}+\operatorname{ran} T_{\phi}\right]\right)^{*}=$ $\frac{\left(\bigwedge_{\alpha=1}^{\gamma}\left[y_{\alpha}+\operatorname{ran} T_{\phi}\right]\right)^{*}}{\operatorname{det}\left(v_{\alpha}^{(n)}, S^{* n+1} y_{\tau}\right)}$, and therefore (1.1) becomes the stated result in Lemma 1.2.

$$
\begin{aligned}
\sigma_{1, T_{\phi} S^{* n+1} R\left(T_{\phi}\right) S^{n+1}=} & \frac{D_{n}\left(T_{\phi}\right)}{\mathbf{G}(\phi)^{n+1} \cdot \operatorname{det}\left(v_{\alpha}^{(n)}, S^{* n+1} y_{\beta}\right)} \\
& \cdot\left(\bigwedge_{\alpha=1}^{\gamma} v_{\alpha}^{(n)}\right) \otimes\left(\bigwedge_{\alpha=1}^{\gamma}\left[y_{\alpha}+\operatorname{ran} T_{\phi}\right]\right)^{*} .
\end{aligned}
$$

The second equation in Lemma 1.2 follows by substitution of the formulas of Proposition 2.1 into the definition of the perturbation vector.

1.2. Bundles, holonomy, Steinberg symbols. Recall the definition of the tame symbol: if $x \in X$, a complex curve in $C^{n}$, and $f$ and $h$ are meromorphic functions at $x$, the function $\left(\frac{f^{\text {order }_{x} h}}{h^{\text {order }_{f}}}\right)$ is of order 0 at $x$ and we can say it has a value at $x$. The value

$$
c_{x}(f, h)=(-1)^{\operatorname{order}_{x} h \cdot \operatorname{order}_{x} f} \cdot\left(\frac{f^{\operatorname{order}_{x} h}}{h^{\operatorname{order}_{x} f}}\right)
$$

is called the tame symbol at $x$.

Beilinson [8] and Deligne [19] investigated the "universal" bundle $(m, \nabla)$ with connection on $C^{*} \times C^{*}$, and $f \cup g$ the pull-back $(f, g)^{*}(m, \nabla)$. It is known that for any Riemann surface $\mathcal{Y}$ there is an element of the group $H^{1}\left(Y, \mathcal{O}_{\mathcal{Y}}^{*}\right)$ obtained from the universal line bundle by the pull-back

$$
r_{0}(f, g)=(f, g)^{*}(m, \nabla) \quad \text { where } \quad(f, g): \mathcal{Y} \rightarrow C^{*} \times C^{*}, \quad x \rightarrow((f(x), g(x)) .
$$

The regulator map $r_{0}$ is associated with the residue map, so that if $S=Z(f) \cup$ $Z(g)$ is a finite subset of $\mathcal{Y}$ there is the long exact Gysin sequence:

$$
\left.0 \rightarrow H^{1}\left(\mathcal{Y}, C^{*}\right) \rightarrow H^{1}(\mathcal{Y}-S), C^{*}\right) \stackrel{\amalg_{\lambda \in S} \partial_{\lambda}}{\longrightarrow} \coprod_{\lambda \in S} C^{*} \rightarrow H^{2}\left(\mathcal{Y}, C^{*}\right) \longrightarrow \cdots
$$

where $\partial_{\lambda}$ is the residue map at $\lambda, \coprod_{\lambda \in S} C^{*}$ denotes the direct product and $\partial_{\lambda} r_{0}(f, g)$ $=c_{\lambda}(f, g)$. 
The bundle construction. For smooth complex valued $\phi$ and $\psi$ defined and non-vanishing on $\mathbb{T}$, the line bundle $\phi \cup \psi$ over the loop $\mathbb{T}$ is defined by specifying transition functions

$$
\left\{\psi^{\frac{1}{2 \pi i}\left(\log _{\alpha} \phi-\log _{\beta} \phi\right)}\right\} \quad \text { together with the connection form } \quad\left\{-\frac{1}{2 \pi i} \log _{\alpha} \phi \cdot \frac{d \psi}{\psi}\right\}
$$

for a Čech cover $\left\{U_{\alpha}\right\}$ such that $\left.\log \phi\right|_{U_{\alpha}}$ is defined (denoted by $\log _{\alpha} \phi$ ). In other words, the relation between local sections $s_{\mu}=\left\{\log _{\mu} \phi, \psi\right\}$ defined by different branches of the logarithm on $U_{\mu} \cap U_{\nu}$ is

$$
\left\{\psi^{\frac{1}{2 \pi i}\left(\log _{\mu} \phi-\log _{\nu} \phi\right)}\right\} \cdot\left\{\log _{\nu} \phi, \psi\right\}=\left\{\log _{\mu} \phi, \psi\right\}
$$

and the connection $\nabla$ on the bundle $E$ defined by these transition functions is characterized by

$$
\nabla(\{\log (\phi), \psi\})=\frac{1}{2 \pi i} \log \phi \cdot \frac{d \psi}{\psi} \otimes\{\log (\phi), \psi\} .
$$

There is an identification $H^{1}\left(\mathbb{T}, C^{*}\right) \cong \operatorname{Hom}\left(\pi_{1}(\mathbb{T}), C^{*}\right) \cong C^{*}$ determined by lifting a generator $\rho$ of $\pi_{1}(\mathbb{T})$ to the bundle $\mathcal{E}$ defined by these transition functions.

Fix base points $p \in \mathbb{T}$ and $\zeta \in C^{*}$ so that $[(p, \zeta)] \in \mathcal{E}$. Let $\rho$ be a closed path in $\mathbb{T}$ starting at $p$ and winding counterclockwise to $p$. Let $\tilde{\rho}$ be a horizontal lift of $\rho$ to $\mathcal{E}$ starting at $[(p, \zeta)]$. Parallel transporting via the connection around a loop leads to $[(p, \tau \cdot \zeta)]$, where $\tau \in C^{*}$. The map $\rho \rightarrow \tau$ defines an element of $\operatorname{Hom}\left(\pi_{1}(\mathbb{T}), C^{*}\right)$.

The holonomy $\tau$ is computed by choosing a disjoint union of open arcs obtained by decomposing $\left\{\phi^{-1}\left(U_{\alpha}\right) \cap \psi^{-1}\left(U_{\beta}\right)\right\}_{\alpha, \beta}$ into a disjoint union of open arcs $\left\{I_{j}^{\alpha, \beta}\right\}_{j=1}^{m(\alpha, \beta)}$ on $\mathbb{T}$ so that each arc $I_{j}$ has a branch $\log _{j}$ of the logarithm defined, and then further refining this union into a covering by open $\operatorname{arcs}\left\{J_{j}\right\}_{j=1}^{n}$, of $\mathbb{T}$, so that $p \in J_{1}$ and $p_{\mu} \in J_{\mu} \cap J_{\mu+1}$ for $2 \leq \mu \leq n-1$ while $p_{n} \in J_{1} \cap J_{n}$. Then as we proceed around the circle counterclockwise we first hit $J_{2}$, then $J_{3}$, etc. Thus for $1 \leq \mu \leq n-1$ we have $J_{\mu} \cap J_{\mu+1} \neq \emptyset$, and $J_{1} \cap J_{n} \neq \emptyset$.

The horizontal lift on $J_{\mu}$ is

$$
\psi\left(p_{\mu+1}\right)^{\frac{1}{2 \pi i}\left[\log _{\mu+1} \phi\left(p_{\mu+1}\right)-\log _{\mu} \phi\left(p_{\mu+1}\right)\right]} \cdot \exp \frac{1}{2 \pi i} \int_{p_{\mu}}^{p_{\mu+1}} \log _{\mu} \phi \frac{d \psi}{\psi},
$$

so that the total lift around $\mathbb{T}$ is

$$
\begin{aligned}
\tau=\prod_{\mu=1}^{n-1}\left(\psi\left(p_{\mu+1}\right)^{\frac{1}{2 \pi i}\left[\log _{\mu+1} \phi\left(p_{\mu+1}\right)-\log _{\mu} \phi\left(p_{\mu+1}\right)\right]} \cdot \exp \frac{1}{2 \pi i} \int_{p_{\mu}}^{p_{\mu+1}} \log _{\mu} \phi \frac{d \psi}{\psi}\right) \\
\times \psi\left(p_{n}\right)^{\left(\frac{1}{2 \pi i} \log _{1} \phi\left(p_{n}\right)-\log _{n} \phi\left(p_{n}\right)\right)} \cdot \exp \left(\frac{1}{2 \pi i} \int_{p_{n}}^{p_{1}} \log _{1} \phi \frac{d \psi}{\psi}\right) .
\end{aligned}
$$

Keeping track of telescoping factors [8, [16] shows that this product can be rewritten:

Theorem 1.4. If the unit circle taken positively is a generator $\Sigma$ of $\pi_{1}(\mathbb{T})$, then for functions $\phi$ and $\psi$ smooth on $\mathbb{T}$, for example in the Krein algebra $K_{2,2}^{\frac{1}{2}, \frac{1}{2}}$, the element $\phi \cup \psi$ of $H^{1}\left(\mathbb{T}, C^{*}\right) \cong \operatorname{Hom}\left(\pi_{1}(\mathbb{T}), C^{*}\right)$ is given by

$$
\phi \cup \psi(\Sigma)=\exp \frac{1}{2 \pi i}\left(\int_{|z|=1} \log \phi \cdot \frac{d \psi}{\psi}-\log \psi(p) \int_{|z|=1} \frac{d \phi}{\phi}\right) .
$$


Here $p$ is a base point of the unit circle $\mathbb{T}$, the branches of the logarithms are continuous except possibly at $p$, and the integrals are taken positively over the circle $\mathbb{T}$ starting at $p$.

The present authors proved an Index Theorem [14, [16] which deals with the Steinberg symbols of equivalence classes of Toeplitz operators modulo the trace ideal and smooth functions $\phi, \psi$ defined on the unit circle $\mathbb{T}$ :

Theorem 1.5. For $\phi, \psi \in K_{2,2}^{\frac{1}{2}, \frac{1}{2}}$, Fredholm $T_{\psi}$ and $T_{\phi}$,

$$
\operatorname{det}_{*} \partial\left\{T_{\phi}+\mathcal{L}^{1}(H), T_{\psi}+\mathcal{L}^{1}(H)\right\}=\phi \cup \psi(\Sigma) .
$$

where $\Sigma$ is the unit circle taken positively.

Theorem 1.5 is applied to give $\operatorname{det}_{*} \partial\left\{T_{\phi}+\mathcal{L}^{1}(H), T_{z^{n+1}}+\mathcal{L}^{1}(H)\right\}=\phi \cup z^{n+1}(\Sigma)$.

Remark 1.3. Taking $p=1$ and using Theorem 1.4 and the Index Theorem 1.5 above we get

$$
\begin{gathered}
\mathbf{G}(\phi)^{n+1} \equiv \operatorname{det}_{*} \partial\left\{T_{\psi z^{\gamma}}+\mathcal{L}^{1}(H), S^{n+1}+\mathcal{L}^{1}(H)\right\}=\psi z^{\gamma} \cup z^{n+1}(\Sigma) \\
=\exp \frac{1}{2 \pi i}\left(\int_{|z|=1} \log \psi z^{\gamma} \cdot \frac{d z^{n+1}}{z^{n+1}}-\log p^{n+1} \int_{|z|=1} \frac{d \psi z^{\gamma}}{\psi z^{\gamma}}\right) .
\end{gathered}
$$

Accordingly, since

$$
\frac{1}{2 \pi i} \oint_{\mathbb{T}} \log z^{\gamma} d \log z=\frac{\gamma}{2 \pi i} \int_{0}^{2 \pi} i \theta d(i \theta)=\pi \gamma i
$$

we have

$$
\begin{aligned}
\mathbf{G}(\phi)^{n+1} & =\phi \cup z^{n+1}(\Sigma)=\operatorname{det}_{*} \partial\left\{T_{\phi}+\mathcal{L}^{1}(H), T_{z^{n+1}}+\mathcal{L}^{1}(H)\right\} \\
& =G(\psi)^{n+1} \cdot \exp [-(n+1) \pi \cdot \gamma i] .
\end{aligned}
$$

The connection of the Steinberg symbols of operator cosets and the geometric mean of an essentially bounded symbol $\phi$ is intrinsic and does not depend on the smoothness of the symbol. A relationship persists even when the smoothness assumptions used here to define the bundle $\phi \cup z^{n+1}$ are not satisfied:

Recall the following result [17, [16. If $\phi \in \mathcal{L}^{\infty}(\mathbb{T})$ and $T_{\phi}$ is Fredholm, then

$$
\left|\operatorname{det}_{*}\left\{T_{\phi}+\mathcal{L}^{1}(H), S^{n+1}+\mathcal{L}^{1}(H)\right\}\right|=\exp \frac{n+1}{2 \pi} \int_{0}^{2 \pi} \log \left|\phi\left(e^{i \theta}\right)\right| d \theta .
$$

The classical Szegö Theorem as a holonomy result. It is of some interest to see now that the original Szegö result may be viewed geometrically:

Remark 1.4. Suppose $\phi \in K_{2,2}^{\frac{1}{2}, \frac{1}{2}} \cap \mathcal{W}$, where $\mathcal{W}$ is the Wiener algebra, and wind $(\phi, 0)=0$. Then with the Wiener- Hopf factorization $\phi=\phi_{+} \phi_{-}$, the foregoing considerations give

$$
\begin{aligned}
\lim _{n \rightarrow \infty} \frac{D_{n}\left(T_{\phi}\right)}{\phi \cup z^{n+1}(\Sigma)} & =\phi_{-} \cup \phi_{+}(\Sigma)=\operatorname{det}_{*} \circ \partial\left\{T_{\phi_{-}}+\mathcal{L}^{1}(H), T_{\phi_{+}}+\mathcal{L}^{1}(H)\right\} \\
& =\exp \sum_{k=1}^{\infty} k\left(\log \phi_{+}\right)_{k}\left(\log \phi_{-}\right)_{-k} .
\end{aligned}
$$


The limit is holonomy when the index is non-zero. Note that for $\gamma \neq 0$, $E(\psi)=\bar{g} \cup f(\Sigma)$ and by definition $G(b)=\frac{\bar{g}}{f} \cup z(\Sigma)$ is a holonomy. The bundles over $\mathbb{T}$ form a group. Thus with $\times$ the group multiplication, the holonomy of the product bundle is

$$
E(\psi) G(b)^{\gamma}=\left(\bar{g} \cup f \times \frac{\bar{g}}{f} \cup z^{\gamma}\right)(\Sigma) .
$$

There is a relationship between the Jordan chains lying over null vectors, and the holonomy of a product bundle.

Proposition 1.2. Let $\phi=\psi z^{\gamma}$ for integer-valued $\gamma>0$ with normalized WienerHopf factorization $\psi=f \bar{g}$, and let $f$ and $g$ be outer functions in $K_{2,2}^{\frac{1}{2}, \frac{1}{2}} \cap \mathcal{W}$. Then

$$
(-1)^{\sum_{\alpha=1}^{\gamma} m_{\alpha}} \frac{\prod_{\lambda \in \sigma\left(T_{\phi} T_{\phi}-1\right) \backslash\{0\}} \lambda}{\operatorname{det}\left(u_{\alpha}, T_{\frac{1}{g}} e_{\tau}\right)_{1 \leq \alpha, \tau \leq \gamma}}=E(\psi) G\left(\frac{\bar{g}}{f}\right)^{\gamma}=\left(\bar{g} \cup f \times \frac{\bar{g}}{f} \cup z^{\gamma}\right)(\Sigma) .
$$

1.3. Inner outer factorization and normalization. We are free to take any factorization for the symbol. There are several reasons for considering the factorization in the form $\phi=\Theta_{1}{ }^{-} \Theta_{2} f \cdot \bar{g}$ with $\Theta_{1}=\prod_{i=1}^{q} \frac{z-\nu_{i}}{1-\bar{\nu}_{i} z}$ and $\Theta_{2}=\prod_{k=1}^{\ell} \frac{z-\mu_{k}}{1-\bar{\mu}_{k} z}$ coprime finite Blaschke products, and $f$ and $g$ outer functions. Among these we note:

- Any $\phi \in \mathcal{L}^{\infty}$ with $\inf |\phi|>0$ factor 3 as $\Theta_{1} \bar{\Theta}_{2} h k$ where $h$ is outer and $k$ is continuous; $T_{\phi}$ is Fredholm iff $T_{\Theta_{1} \bar{\Theta}_{2}}$ is Fredholm and index $T_{\phi}=\operatorname{index} T_{\Theta_{1} \bar{\Theta}_{2}}$. This factorization 4 is unique (up to constants) when $k=1$.

- The factorization is unique if $\phi$ is rational.

- Factoring $\phi$ into $\Theta_{1} \bar{\Theta}_{2} f \bar{g}$ defines a generator of $\operatorname{det} H\left(T_{\phi} T_{\phi^{-1}}\right)$ as follows: pick a basis $\left\{x_{j}\right\}$ of $\operatorname{ker} T_{\Theta_{1} \bar{\Theta}_{2}}$. The tensor product $\bigwedge T_{f} x_{j} \otimes\left(T_{\frac{1}{g}} x_{j}+\operatorname{ran} T_{\phi}\right)^{*}$ is a non-zero element of $\operatorname{det}\left(T_{\phi} T_{\phi^{-1}}\right)$ which does not depend on the choice of $\left\{x_{j}\right\}$. Pairing with the perturbation vector produces the coefficient

$$
\frac{(-1)^{\sum_{\alpha=1}^{\gamma} m_{\alpha}} \prod_{\lambda \in \sigma\left(T_{\phi} T_{\phi}-1\right) \backslash\{0\}} \lambda\left\|\wedge T_{\frac{1}{g}} \mid \operatorname{ker} T_{\Theta_{1} \bar{\Theta}_{2}}\right\|^{2}}{\operatorname{det}\left(u_{\alpha}, T_{\frac{1}{g}} e_{\tau}\right)_{1 \leq \alpha, \tau \leq \gamma}},
$$

where $\left\{u_{\alpha}\right\}$ are the top root vectors associated with $\left\{T_{f} x_{a}\right\}$.

There are two prominent generators for $\operatorname{det} H\left(T_{\phi} S^{* n+1} R\left(T_{\phi}\right) S^{n+1}\right)$, if $D_{n}\left(T_{\phi}\right) \neq$ 0 . One is the perturbation vector $\sigma_{1, T_{\phi}} S^{* n+1} R\left(T_{\phi}\right) S^{n+1}$ and the other is $\bigwedge w_{j}^{n} \otimes$ $\bigwedge\left(S^{n+1} w_{j}^{n}+\operatorname{ran} T_{\phi}\right)^{*}$, where $w_{j}^{n}$ is any basis for $\operatorname{ker} S^{* n+1} R\left(T_{\phi}\right) S^{n+1}$. Again, we have independence of the choice of basis. Pairing these two yields the Szegö sequence $\frac{D_{n}\left(T_{\phi}\right)}{\mathbf{G}(\phi)^{n+1}}$, with the generalized geometric mean in the denominator.

- When $\phi$ is both unimodular and rational we have previously [17 explored a relation between the geometry of subspaces in Hilbert space and hyperbolic plane geometry by evaluating the Szegö limit in terms of the Bolyai-Lobachevsky angles of parallelism associated with pairs of zeros of the Blaschke factors. Recall that these angles are defined by limiting directions.

3 N.K. Nikol'skii, Treatise on the shift operator, Springer-Verlag, Grundlehren 273, Berlin (1986).

${ }^{4}$ It is natural to consider the Szegö limit problem for more general inner functions. For example, when $\Theta_{1}$ is singular and the supports of $\Theta_{1}$ and $\Theta_{2}$ are identical, as in the work of Lee and Sarason (The spectra of some Toeplitz operators, J. Anal. Appl. 33 (1971), 529-543) where $\Theta_{1}$ is singular with mass one and $\Theta_{2}$ is a Blaschke product with zeros clustering at 1. 
- The limit result for the factorization $\phi=\Theta_{1} \bar{\Theta}_{2} f \bar{g}$ clearly displays the way in which the perturbation vector is related to algebraic K-theory, Steinberg symbols, and the tame symbols formed from the zeros of the symbols.

Steinberg symbols were originally introduced for the study of arithmetic because of their natural connection to tame symbols. We are interested in another relationship to geometry in Hilbert space involving the notion of separation of subspaces when degree $\Theta_{1}=\operatorname{degree} \Theta_{2}$. The purely geometric object $\chi^{2}\left(P\left(T_{\bar{\Theta}_{1}}\right), P\left(T_{\bar{\Theta}_{2}}\right)\right) \equiv \operatorname{det}\left(\left.P\left(T_{\bar{\Theta}_{1}}\right) P\left(T_{\bar{\Theta}_{2}}\right)\right|_{\text {ker } T_{\bar{\Theta}_{1}}}\right)$ can be computed in terms of tame symbols and was found to express angular relationships between the indicated kernel spaces (see [15]).

Theorem 1.6. Let $\phi=\Theta_{1} \cdot \bar{\Theta}_{2} f \cdot \bar{g}$ with $\Theta_{1}=\prod_{i=1}^{q} \frac{z-\nu_{i}}{1-\bar{\nu}_{i} z}$ and $\Theta_{2}=\prod_{k=1}^{\ell} \frac{z-\mu_{k}}{1-\bar{\mu}_{k} z}$ coprime finite Blaschke products, and let $f$ and $g$ be outer functions in $K_{2,2}^{\frac{1}{2}, \frac{1}{2}} \cap \mathcal{W}$, the intersection of the Krein algebra $\left\{a \in \mathcal{L}^{\infty}(\mathbb{T}): \sum_{n \in Z}(|n|+1)\left|a_{n}\right|^{2}<\infty\right.$ and the Wiener algebra, $\mathcal{W}$. Let $\gamma \equiv q-\ell=\operatorname{wind}(\phi, 0) \geq 0$. Let $w_{\alpha}=T_{f} x_{\alpha}$ and let $u_{\alpha}$ be the associated top root vectors lying over $(-1)^{m_{\alpha}} T_{f} x_{\alpha}$ where $\left\{x_{\alpha}\right\}_{1}^{\gamma}$ is an orthonormal basis for $\operatorname{ker} T_{\bar{\Theta}_{1} \Theta_{2}}=T_{\bar{\Theta}_{2}}\left(K_{\Theta_{1}} \cap K_{\Theta_{2}}^{\perp}\right)$. Then for any sequence of vectors $\left\{v_{\alpha}^{(n)}\right\}_{1}^{\gamma}$ in $\operatorname{ker} S^{* n+1} R\left(T_{\phi}\right) S^{n+1}$ for which $\lim _{n \rightarrow \infty} v_{\alpha}^{(n)}=T_{f} x_{\alpha}$, for $n \gg 0$,

$$
\begin{aligned}
D_{n}\left(T_{\phi}\right)= & \mathbf{G}(\phi)^{n+1} \cdot \operatorname{det}\left(v_{\alpha}^{(n)}, S^{* n+1} T_{\frac{1}{g}} x_{\tau}\right)_{\gamma \times \gamma} \\
& \cdot\left[\frac{\prod_{\lambda \in \sigma\left(T_{\phi} T_{\phi}-1\right) \backslash\{0\}}^{\lambda}}{\operatorname{det}\left(u_{\alpha}, T_{\frac{1}{g}} x_{\tau}\right)}\right]\left[1+O\left(n^{(1-2 \beta)}\right)\right] .
\end{aligned}
$$

ii)

$$
\begin{aligned}
\lim _{n \rightarrow \infty} & \frac{D_{n}\left(T_{\phi}\right)}{\mathbf{G}(\phi)^{n+1} \cdot \operatorname{det}\left(v_{\alpha}^{(n)}, S^{* n+1} T_{\frac{1}{g}} x_{\tau}\right)} \\
= & \chi^{2}\left(P\left(T_{\bar{\Theta}_{1}}\right), P\left(T_{\bar{\Theta}_{2}}\right)\right) \cdot \prod_{k=1}^{\ell}\left[\frac{f\left(\mu_{k}\right)}{\bar{g}\left(\mu_{k}\right)}\right] \prod_{i=1}^{q}\left[\frac{\bar{g}\left(\nu_{i}\right)}{f\left(\nu_{i}\right)}\right] \\
& \cdot \exp \sum_{k=1}^{\infty} k(\log f)_{k}(\log \bar{g})_{-k} \cdot \operatorname{det}\left(P\left(T_{\bar{\Theta}_{1}}\right) \wedge \operatorname{rp}\left(T_{\Theta_{2}}\right)\right. \\
& \left.\cdot T_{|f|^{2} \mid K_{\Theta_{1}} \cap K_{\Theta_{2}}}\right)^{\frac{1}{2}} \cdot \operatorname{det}\left(P\left(T_{\bar{\Theta}_{1}}\right) \wedge \operatorname{rp}\left(T_{\Theta_{2}}\right) \cdot T_{\left|\frac{1}{g}\right|^{2}} \mid K_{\Theta_{1}} \cap K_{\Theta_{2}}^{\perp}\right)^{\frac{1}{2}}
\end{aligned}
$$

where explicitly for simple zeros $\left\{\mu_{\alpha}\right\}$ and $\left\{\nu_{\tau}\right\}$,

$$
\begin{aligned}
& \chi^{2}\left(P\left(T_{\bar{\Theta}_{1}}\right), P\left(T_{\bar{\Theta}_{2}}\right)\right)=\frac{\prod_{1 \leq \alpha, \tau \leq \ell}\left|1-\bar{\mu}_{\alpha} \mu_{\tau}\right|}{\prod_{\substack{1 \leq \alpha \leq \ell \\
1 \leq \beta \leq q}}\left|1-\bar{\mu}_{\alpha} \nu_{\tau}\right|^{2}} \\
& \cdot \mid \sum_{N} \frac{\prod_{\substack{\mu_{\alpha} \in \bar{N} \\
1 \leq \beta \leq q}}\left(\mu_{\alpha}-\nu_{\tau}\right)}{\prod_{\substack{\mu_{\alpha} \in N \\
\mu_{\tau} \in \bar{N}}}\left(\mu_{\alpha}-\mu_{\tau}\right)} \cdot \frac{\prod_{\frac{1}{\bar{\mu}_{\alpha} \in N}}\left(\bar{\mu}_{\alpha}-\bar{\nu}_{\tau}\right)}{\prod_{\substack{1 \leq \tau \leq q \\
\frac{1}{\mu_{\alpha}} \in N \\
\frac{1}{\bar{\mu}_{\tau}} \in \bar{N}}}\left(\bar{\mu}_{\alpha}-\bar{\mu}_{\tau}\right)}
\end{aligned}
$$

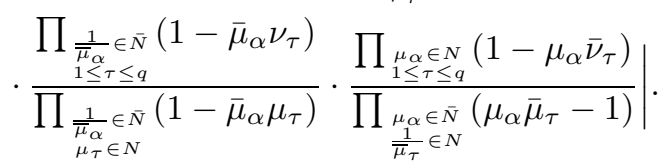


The sum is extended over all subsets $N \subset\left\{\mu_{1}, \cdots, \mu_{\ell} ; \frac{1}{\bar{\mu}_{1}}, \cdots, \frac{1}{\bar{\mu}_{\ell}}\right\}$ of cardinality $\ell$, and $\bar{N}=\left\{\mu_{1}, \cdots, \mu_{\ell} ; \frac{1}{\bar{\mu}_{1}}, \cdots, \frac{1}{\bar{\mu}_{\ell}}\right\} \backslash N$.

For an inner function $\Theta$ we use the notation $K_{\Theta}=H^{2} \ominus \Theta H^{2}$. The numbers $\operatorname{det}\left(P\left(T_{\bar{\Theta}_{1}}\right) \wedge \operatorname{rp}\left(T_{\Theta_{2}}\right) \cdot T_{|f|^{2}} \mid K_{\Theta_{1}} \cap K_{\Theta_{2}}^{\perp}\right)^{\frac{1}{2}}$ and $\operatorname{det}\left(P\left(T_{\bar{\Theta}_{1}}\right) \wedge \operatorname{rp}\left(T_{\Theta_{2}}\right) \cdot T_{\left|\frac{1}{g}\right|^{2} \mid K_{\Theta_{1}} \cap K_{\Theta_{2}}^{\perp}}\right)^{\frac{1}{2}}$ are fixed finite order determinants. These normalization factors are computed from the eigenvectors associated with the zeros.

\section{Perturbation vectors}

Let $H$ be an $n$-dimensional vector space over a field $F$. Let $T$ be an endomorphism on $H$. Let $p=\operatorname{dim} \operatorname{ker} T$. Then we have the exact sequence

$$
\mathcal{T}: \quad 0 \longrightarrow \operatorname{Ker} T \stackrel{i}{\longrightarrow} H \stackrel{T}{\longrightarrow} H \stackrel{\pi_{T}}{\longrightarrow} \text { Coker } T \longrightarrow 0 .
$$

The torsion vector of this complex is the element in $\operatorname{det} \mathcal{T}$ defined as follows: Pick non-zero vectors $s_{1} \in \operatorname{det} \operatorname{ker} T, s_{2} \in \bigwedge^{n-p} H$ and $s_{3} \in \bigwedge^{p} H$, where $s_{1} \wedge s_{2} \neq 0$ and $T s_{2} \wedge s_{3} \neq 0$. Then

$$
(-1)^{p(n-p)} s_{1}^{*} \otimes\left(s_{1} \wedge s_{2}\right) \otimes\left(T s_{2} \wedge s_{3}\right)^{*} \otimes \pi_{T} s_{3}
$$

defines a generator $\sigma(T)$ of $\operatorname{det} \mathcal{T}$ that is independent of the choice of vectors $s_{1}, s_{2}, s_{3}$.

In the same way, a pair of maps $S$ and $T$ acting between finite dimensional spaces $H_{1}$ and $H_{2}$ produces torsion vectors $\sigma(S)$ and $\sigma(T)$. The product

$$
\sigma_{S, T} \equiv \sigma(S) \otimes \sigma(T)^{*}
$$

is then a well-defined element in $\operatorname{det} \mathcal{S} \otimes(\operatorname{det} \mathcal{T})^{*}$.

In infinite dimensions, $\sigma(S)$ and $\sigma(T)$ are not defined, so the coupling represented by $\sigma_{S, T}$ may not factor. But in [15] it was shown how the construction extends to the case where $H_{1}$ and $H_{2}$ are infinite dimensional, with $S$ and $T$ algebraically Fredholm and $S-T$ finite rank, or in the case of Banach spaces where $S$ and $T$ are Fredholm in the usual sense and $S-T$ is nuclear.

The following properties of the norms of perturbation vectors were proved in [15]:

Let $H_{1}$ and $H_{2}$ be Hilbert spaces. Then

i) $\left\|\sigma_{T, S}\right\|=\left\|\sigma_{S, T}\right\|^{-1}$.

ii) If index $T \geq 0$, then $\left\|\sigma_{T, S}\right\|^{2}=\operatorname{det}\left(\left(T T^{*}+P\left(T^{*}\right)\right)^{-1} \cdot\left(S S^{*}+P\left(S^{*}\right)\right)\right)$.

iii) If index $T \leq 0$, then $\left\|\sigma_{T, S}\right\|^{2}=\operatorname{det}\left(\left(T^{*} T+P(T)\right)^{-1}\left(S^{*} S+P(S)\right)\right)$.

iv) Suppose $H_{1}=H_{2}=H$ is a complex Hilbert space and $T^{*} T-T T^{*} \in \mathcal{L}^{1}(H)$. Then $\left\|\sigma_{T, S}\right\|=\left\|\sigma_{T^{*}, S^{*}}\right\|$.

v) $\sigma_{S, T}=\sigma_{T, S}^{*}, \sigma_{T, S} \otimes \sigma_{S, R}=\sigma_{T, R}$.

The equality in (v) is to be understood in the sense that the vectors on the right and left-hand sides are images of each other under the canonical isomorphism $H \otimes H^{*} \cong C$.

Example. Suppose $H=H_{1}=H_{2}$ is a separable Hilbert space and $1-X$ is a trace class operator. Let $x$ be any vector in $\operatorname{det} \operatorname{ker} X$. Then $x$ is decomposable and there is a basis $\left\{x_{j}\right\}$ for $\operatorname{ker} X$ so that $x=\bigwedge x_{j}$. Let $\left\{u_{j}\right\}$ be a set of root vectors of maximal height lying over $\left\{(-1)^{m_{j}} x_{j}\right\}$ relative to $X{\mid \cup_{n=1}^{\infty} \operatorname{ker}(X)^{n}}$ with algebraic 
multiplicity $m_{j}+1$. Recall that the Riesz-Schauder theorem implies there is a direct sum decomposition of the Hilbert space $H=\mathcal{X}+\mathcal{Y}$ into $X$ invariant subspaces, $\mathcal{X}=\bigcap_{n=1}^{\infty} \operatorname{ran}(X)^{n}$ and $\mathcal{Y}=\bigcup_{n=1}^{\infty} \operatorname{ker}(X)^{n}$, of $X$ so that $X$ restricted to $\mathcal{X}$ is invertible, $\mathcal{Y}$ is finite dimensional, and $X$ restricted to $\mathcal{Y}$ is nilpotent. Furthermore,

$$
\sigma_{1, X}=\prod_{\lambda \in \sigma(X) \backslash\{0\}} \lambda \cdot \bigwedge x_{j} \otimes\left(\bigwedge\left(u_{j}+\operatorname{ran} X\right)\right)^{*}
$$

and

$$
\left\|\sigma_{1, X}\right\|=\frac{\prod_{\lambda \in \sigma(X) \backslash\{0\}}|\lambda|}{\left|\operatorname{det}\left(u_{i}, y_{j}\right)\right|}=\prod_{s_{\alpha}(X) \neq 0} \mathbf{s}_{\alpha}(X),
$$

where $\left\{y_{j}\right\}$ is any orthonormal basis for $\operatorname{ker} X^{*},\left\{u_{i}\right\}$ is any set of maximal root vectors relative to any orthonormal basis of $\operatorname{ker} X$ and $\left\{\mathbf{s}_{\alpha}(\mathbf{X})\right\}$ are the singular values of $X$. If $\sigma(X) \backslash\{0\}=\emptyset$, the product is understood to be 1 .

Proposition 2.1. $\quad$ Let $X$ be an operator on Hilbert space $H$ so that $X=i$ dentity + trace class. Suppose $\left\{w_{\alpha}\right\}_{1}^{\gamma}$ is any basis for $\operatorname{ker} X$ and $\left\{y_{\alpha}\right\}_{1}^{\gamma}$ is any orthonormal basis for $\operatorname{ker} X^{*}$. Let $\left\{u_{\alpha}\right\}_{1}^{\gamma}$ be a corresponding set of maximal root vectors for $\left\{w_{\alpha}\right\}_{1}^{\gamma}$, i.e., $(X)^{\max } u_{\alpha}=w_{\alpha}, \alpha=1,2, \ldots, \gamma$. Then

i) $\sigma_{1, X}=(-1)^{\sum_{\alpha=1}^{\gamma} m_{\alpha}} \cdot \prod_{\lambda \in \sigma(X) \backslash\{0\}} \lambda \cdot\left(\bigwedge_{k=1}^{\gamma} w_{k}\right) \otimes\left(\bigwedge_{\tau=1}^{\gamma}\left[u_{\tau}+\operatorname{ran} X\right]\right)^{*}$,

ii) $(-1)^{\sum_{\alpha=1}^{\gamma} m_{\alpha}} \cdot \prod_{\lambda \in \sigma(X) \backslash\{0\}} \lambda \cdot \frac{\left\|\bigwedge w_{j}\right\|^{2}}{\operatorname{det}\left(u_{j}, y_{i}\right)}=\operatorname{det}\left(X+\sum_{j=1}^{\gamma} y_{j} \otimes w_{j}\right)$.

iii) Let $\left\{u_{\alpha}^{\prime}\right\}_{1}^{\gamma}$ be the maximal root vectors corresponding to $\left\{w_{\alpha}^{\prime}\right\}_{1}^{\gamma}$, the orthonormal vectors obtained from $\left\{w_{\alpha}\right\}_{1}^{\gamma}$ by the Gram-Schmidt process. Then $\left\|\bigwedge_{\alpha=1}^{\gamma} w_{\alpha}\right\| \cdot$ $\operatorname{det}\left(u_{\alpha}^{\prime}, y_{\tau}\right)=\operatorname{det}\left(u_{\alpha}, y_{\tau}\right)$.

Proof. By definition of the perturbation vector $\sigma_{1, X}$ (see $\S 2$ in [15]), we have

$$
\sigma_{1, X}=\operatorname{det}\left(X+\sum_{j=1}^{\gamma} u_{j} \otimes w_{j}\right)\left(\bigwedge_{1}^{\gamma} t_{j}\right) \otimes\left(\bigwedge_{1}^{\gamma}\left(u_{j}+\operatorname{ran} X\right)\right)^{*},
$$

where ${ }^{*}$ denotes the dual vector and $\left\{t_{j}\right\}_{1}^{\gamma}$ is a dual basis to $\left\{w_{j}\right\}_{1}^{\gamma}$, i.e., $\left(t_{j}, w_{k}\right)=\delta_{j k}$ and $\left\{t_{j}\right\}_{1}^{\gamma}$ spans $\operatorname{ker} X$. Note that $\bigwedge_{1}^{\gamma} t_{j}=\left\|\bigwedge_{1}^{\gamma} w_{j}\right\|^{-2} \bigwedge_{1}^{\gamma} w_{j}$.

But

$$
\operatorname{det}\left(X+\sum u_{j} \otimes w_{j}\right)=\operatorname{det}\left(X_{\left.\right|_{\mathcal{X}}}\right) \operatorname{det}\left(X+\sum u_{j} \otimes w_{\left.j\right|_{\mathcal{Y}}}\right) \text {, }
$$

and

$$
\begin{gathered}
\operatorname{det}\left(X_{\left.\right|_{\mathcal{X}}}\right)=\prod_{\lambda \in \sigma(X) \backslash\{0\}} \lambda, \\
\operatorname{det}\left(X+\sum_{1}^{\gamma} u_{j} \otimes w_{\left.j\right|_{\mathcal{Y}}}\right)=(-1)^{\sum_{j=1}^{\gamma} m_{j}}\left\|\bigwedge_{1}^{\gamma} w_{j}\right\|^{2} .
\end{gathered}
$$

Thus i) follows by substitution.

Now we prove ii). Again by definition of the perturbation vector $\sigma_{1, X}$, we have

$$
\sigma_{1, X}=\operatorname{det}\left(X+\sum_{j=1}^{\gamma} y_{j} \otimes w_{j}\right)\left(\bigwedge_{1}^{\gamma} t_{j}\right) \otimes\left(\bigwedge_{1}^{\gamma}\left(y_{j}+\operatorname{ran} X\right)\right)^{*}
$$


With $u_{j}=\sum_{k=1}^{\gamma}\left(u_{j}, y_{k}\right) y_{k} \bmod \operatorname{ran} X$ we have

$$
\left(\bigwedge_{1}^{\gamma}\left(u_{j}+\operatorname{ran} X\right)^{*}=\frac{\left(\bigwedge_{1}^{\gamma}\left(y_{j}+\operatorname{ran} X\right)\right)^{*}}{\operatorname{det}\left(u_{j}, y_{k}\right)} .\right.
$$

Therefore,

$$
\frac{\operatorname{det}\left(X+\sum_{1}^{\gamma} u_{j} \otimes w_{j}\right)}{\operatorname{det}\left(u_{j}, y_{k}\right)}=\operatorname{det}\left(X+\sum_{j=1}^{\gamma} y_{j} \otimes w_{j}\right)
$$

so that

$$
\left[(-1)^{\sum_{\alpha=1}^{\gamma} m_{\alpha}} \prod_{\lambda \in \sigma(X \backslash\{0\}} \lambda\right] \frac{\left\|\bigwedge_{1}^{\gamma} w_{j}\right\|^{2}}{\operatorname{det}\left(u_{j}, y_{k}\right)}=\operatorname{det}\left(X+\sum_{1}^{\gamma} y_{j} \otimes w_{j}\right) .
$$

Next we prove iii). Replacing $\left\{w_{j}\right\}_{1}^{\gamma}$ by $\left\{w_{j}^{\prime}\right\}_{1}^{\gamma}$ and $\left\{u_{j}\right\}_{1}^{\gamma}$ by $\left\{u_{j}^{\prime}\right\}_{1}^{\gamma}$ in part ii) gives

$$
\frac{(-1)^{\sum_{\alpha=1}^{\gamma} m_{\alpha}} \prod_{\lambda \in \sigma(X \backslash\{0\}} \lambda}{\operatorname{det}\left(u_{j}^{\prime}, y_{k}\right)}=\operatorname{det}\left(X+\sum_{1}^{\gamma} y_{j} \otimes w_{j}^{\prime}\right) .
$$

By equating expressions for the perturbation vector, we get

$$
\begin{aligned}
& \operatorname{det}\left(X+\sum_{1}^{\gamma} y_{j} \otimes w_{j}^{\prime}\right)\left(\bigwedge_{1}^{\gamma} w_{j}^{\prime}\right) \otimes\left(\bigwedge_{1}^{\gamma}\left(y_{j}+\operatorname{ran} X\right)^{*}\right. \\
= & \operatorname{det}\left(X+\sum_{1}^{\gamma} y_{j} \otimes w_{j}\right)\left(\bigwedge_{1}^{\gamma} t_{j}\right) \otimes\left(\bigwedge_{1}^{\gamma}\left(y_{j}+\operatorname{ran} X\right)\right)^{*} .
\end{aligned}
$$

Again $\bigwedge_{1}^{\gamma} t_{j}=\left\|\bigwedge_{1}^{\gamma} w_{j}\right\|^{-2} \bigwedge_{1}^{\gamma} w_{j}$ and $\bigwedge_{1}^{\gamma} w_{j}^{\prime}=\left\|\bigwedge_{1}^{\gamma} w_{j}\right\|^{-1} \bigwedge_{1}^{\gamma} w_{j}$. Therefore,

$$
\operatorname{det}\left(X+\sum_{1}^{\gamma} y_{j} \otimes w_{j}^{\prime}\right)=\left\|\bigwedge_{1}^{\gamma} w_{j}\right\|^{-1} \operatorname{det}\left(X+\sum_{1}^{\gamma} y_{j} \otimes w_{j}\right) .
$$

Consequently,

$$
\begin{aligned}
&(-1)^{\sum_{\alpha=1}^{\gamma} m_{\alpha}} \prod_{\lambda \in \sigma(X) \backslash\{0\}} \lambda \cdot \frac{1}{\operatorname{det}\left(u_{j}^{\prime}, y_{k}\right)} \\
&=\left\|\bigwedge_{1}^{\gamma} w_{j}\right\|^{-1}\left[(-1)^{\sum_{\alpha=1}^{\gamma} m_{\alpha}} \prod_{\lambda \in \sigma(X) \backslash\{0\}} \lambda\right] \frac{\left\|\bigwedge_{1}^{\gamma} w_{j}\right\|^{2}}{\operatorname{det}\left(u_{j}, y_{k}\right)} .
\end{aligned}
$$

Canceling like factors gives iii).

\section{Proof of Theorem 1.1, part i)}

The proof of Theorems 1.1, 1.2, and 1.3 are centered around equation (35) of [15] cited earlier:

$$
\frac{D_{n}\left(T_{\phi}\right)}{\mathbf{G}(\phi)^{n+1}}=\operatorname{det}\left(T_{\phi} S^{* n+1} R\left(T_{\phi}\right) S^{n+1}+P\left(T_{\bar{\phi}}\right) S^{n+1}\right) .
$$

We proceed under the assumptions of Theorem 1.1, part i). In particular, since $T_{\phi} T_{\phi^{-1}}$ is of the form $1+J$, where $J$ is in the trace ideal, $T_{\phi^{-1}}$ is a regularizer for $T_{\phi}$ and $R\left(T_{\phi}\right)-T_{\phi^{-1}}$ is in $\mathcal{L}^{1}(H)$. Let $\gamma=\operatorname{Index} T_{\phi}=\operatorname{dim} \operatorname{ker} T_{\bar{\phi}} \geq 1$. Thus $P\left(T_{\bar{\phi}}\right)$ has rank $\gamma$. 
Lemma 1.1 of $\S 1.1$ is contained in the following lemma.

Lemma 3.1. Suppose $\phi \in \mathcal{L}^{\infty}(\mathbb{T})$ and $T_{\phi}$ is injective and Fredholm with $T_{\phi} T_{\phi^{-1}}-$ $1 \in \mathcal{L}^{1}\left(H^{2}(\mathbb{T})\right)$. Then as $n \rightarrow \infty, P\left(S^{* n+1} R\left(T_{\phi}\right) S^{n+1}\right)$ converges to $P\left(T_{\phi^{-1}}\right)$ in trace norm and $D_{n}\left(T_{\phi}\right)=0 \Longleftrightarrow \operatorname{det}\left(S^{n+1} v_{\alpha}^{(n)}, y_{\tau}\right)=0$, where $\left\{v_{\alpha}^{(n)}\right\}_{1}^{\gamma}$ (respectively $\left.\left\{y_{\tau}\right\}_{1}^{\gamma}\right)$ is a basis for $\operatorname{ker}\left(S^{* n+1} R\left(T_{\phi}\right) S^{n+1}\right)$ (respectively $\operatorname{ker}\left(T_{\bar{\phi}}\right)$ ).

Proof. Since $T_{\phi} T_{\phi^{-1}}-1$ is compact, $R\left(T_{\phi}\right)$ has the form $T_{\phi^{-1}}+F$ where $F$ is also compact. Consequently, $S^{* n+1} R\left(T_{\phi}\right) S^{n+1}$ converges in the uniform norm to $T_{\phi^{-1}}$. Since $T_{\phi^{-1}}$ is surjective, the same is true for $S^{* n+1} R\left(T_{\phi}\right) S^{n+1}$ provided that $n \gg 0$. The first assertion now follows since $P\left(S^{* n+1} R\left(T_{\phi}\right) S^{n+1}\right)=1-$ $S^{* n+1} R\left(T_{\phi}\right)^{*} S^{n+1}\left[S^{* n+1} R\left(T_{\phi}\right) S^{n+1} S^{* n+1} R\left(T_{\phi}\right)^{*} S^{n+1}\right]^{-1} S^{* n+1} R\left(T_{\phi}\right) S^{n+1}$.

Now index $S^{* n+1} R\left(T_{\phi}\right) S^{n+1}=\gamma$ and therefore dim ker $S^{* n+1} R\left(T_{\phi}\right) S^{n+1}=\gamma$. Now fix $n \gg 0$. When $D_{n}(\phi) \neq 0$ we have from Proposition 1.1

$$
\frac{D_{n}\left(T_{\phi}\right)}{\mathbf{G}(\phi)^{n+1}}=\operatorname{det}\left(T_{\phi} S^{* n+1} R\left(T_{\phi}\right) S^{n+1}+P\left(T_{\bar{\phi}}\right) S^{n+1}\right)
$$

If we replace $\phi$ by $\phi-\lambda$, then continuity in the generating symbol implies that this equation holds also when $D_{n}\left(T_{\phi}\right)=0$. Thus $D_{n}\left(T_{\phi}\right)=0$ if and only if there is a non-zero vector $x \in \operatorname{ker}\left(S^{* n+1} R_{\phi} S^{n+1}\right)$ and $P\left(T_{\bar{\phi}}\right) S^{n+1} x=0$. For $n \gg 0$ this means $x$ is a linear combination $x=\sum_{\alpha=1}^{\gamma} \lambda_{\alpha} v_{\alpha}^{(n)}$ and $\left(S^{n+1} x, y_{\tau}\right)=0$ for all $\tau$. By Cramer's rule this is the same as $\operatorname{det}\left(\left(S^{n+1} v_{\alpha}^{(n)}, y_{\tau}\right)\right)=0$.

Lemma 3.2. Suppose that $\left(\begin{array}{ll}A & B \\ C & D\end{array}\right)$ and $A$ are invertible, and are of the form $1+$ trace class. Then

$$
\operatorname{det}\left(\begin{array}{ll}
A & B \\
C & D
\end{array}\right)=\frac{\operatorname{det} A}{\operatorname{det}\left(\begin{array}{ll}
0 & 0 \\
0 & 1
\end{array}\right)\left(\begin{array}{ll}
A & B \\
C & D
\end{array}\right)^{-1} \mid \operatorname{ran}\left(\begin{array}{ll}
0 & 0 \\
0 & 1
\end{array}\right)} .
$$

Proof. We have $\left(\begin{array}{cc}A & B \\ C & D\end{array}\right)=\left(\begin{array}{cc}1 & 0 \\ C A^{-1} & 1\end{array}\right) \cdot\left(\begin{array}{cc}A & B \\ 0 & -C A^{-1} B+D\end{array}\right)$. Taking determinants we have

$$
\operatorname{det}\left(\begin{array}{ll}
A & B \\
C & D
\end{array}\right)=\operatorname{det} A \cdot \operatorname{det}\left(-C A^{-1} B+D\right) \text {. }
$$

Now (cf. Lemma 2.9 in [12] $), D-C A^{-1} B=\left(\left(\begin{array}{ll}0 & 0 \\ 0 & 1\end{array}\right)\left(\begin{array}{ll}A & B \\ C & D\end{array}\right)^{-1} \mid \operatorname{ran}\left(\begin{array}{ll}0 & 0 \\ 0 & 1\end{array}\right)\right)^{-1}$. Hence

$$
\operatorname{det}\left(\begin{array}{ll}
A & B \\
C & D
\end{array}\right)=\frac{\operatorname{det} A}{\operatorname{det}\left(\begin{array}{ll}
0 & 0 \\
0 & 1
\end{array}\right)\left(\begin{array}{ll}
A & B \\
C & D
\end{array}\right)^{-1} \mid \operatorname{ran}\left(\begin{array}{ll}
0 & 0 \\
0 & 1
\end{array}\right)} .
$$

The Riesz Schauder decomposition applies to $X=T_{\phi} T_{\phi^{-1}}$ so that $H^{2}(\mathbb{T})=$ $\mathcal{X}+\mathcal{Y}$ where $\mathcal{X}=\bigcap_{n=1}^{\infty} \operatorname{ran}\left(T_{\phi} T_{\phi^{-1}}\right)^{n}$ and $\mathcal{Y}=\bigcup_{n=1}^{\infty} \operatorname{ker}\left(T_{\phi} T_{\phi^{-1}}\right)^{n}$. Let $P_{\mathcal{X}}$ be the projection onto $\mathcal{X}=\bigcap_{n=1}^{\infty} \operatorname{ran}(X)^{n}$ parallel to $\mathcal{Y}$, and set $P_{\mathcal{Y}}=1-P_{\mathcal{X}}$.

Then we have a block matrix decomposition

$$
X_{n}=\left(\begin{array}{ll}
A & B \\
C & D
\end{array}\right) \equiv T_{\phi} S^{* n+1} R\left(T_{\phi}\right) S^{n+1}+P\left(T_{\bar{\phi}}\right) S^{n+1}
$$

Since $R\left(T_{\phi}\right)=T_{\phi^{-1}}-E$ where $E$ is trace class, $X_{n}$ converges to $T_{\phi} T_{\phi^{-1}}$ in the uniform norm. It then follows that $P(\mathcal{X}) X_{\left.n\right|_{\mathcal{X}}}$ is invertible provided that $n \gg 0$. 
Now suppose that $n \gg 0$ is chosen so that $X_{n}$ is invertible, e.g., $D_{n}\left(T_{\phi}\right) \neq 0$. Then by Proposition 1.1 and Lemma 3.2

$$
\frac{D_{n}\left(T_{\phi}\right)}{\mathbf{G}(\phi)^{n+1}}=\frac{\operatorname{det}\left(\left.P_{\mathcal{X}} X_{n}\right|_{\mathcal{X}}\right)}{\operatorname{det}\left(\left.P_{\mathcal{Y}} X_{n^{-1}}\right|_{\mathcal{Y}}\right)}
$$

Since the numerator of this quotient converges to

$$
\operatorname{det}\left(\left.P_{\mathcal{X}} T_{\phi} T_{\phi^{-1}}\right|_{\mathcal{X}}\right)=\prod_{\lambda \in \sigma\left(T_{\phi} T_{\phi^{-1}}\right) \backslash\{0\}} \lambda
$$

the product of the non-zero eigenvalues - using the well-known theorem of V.B. Lidskii - it suffices to consider the denominator $\operatorname{det}\left(\left.P_{\mathcal{Y}} X_{n^{-1}}\right|_{\mathcal{Y}}\right)$.

Choose a basis $\left\{w_{\alpha}\right\}_{1}^{\gamma}$ for the kernel of $T_{\phi^{-1}}$. Let $u_{1}, u_{2}, \ldots, u_{\gamma}$ be associated vectors so that $u_{\alpha}$ has maximal order with respect to $\left\{w_{\alpha}\right\}$, i.e., $\left(T_{\phi} T_{\phi^{-1}}\right)^{m_{\alpha}} u_{\alpha}$ $=w_{\alpha}$ where $m_{\alpha}=\max \left\{m: \exists x\right.$ so that $\left.\left(T_{\phi} T_{\phi^{-1}}\right)^{m} x=w_{\alpha}\right\}$. Then the collection $\left\{\left(T_{\phi} T_{\phi^{-1}}\right)^{r} u_{\alpha}: 0 \leq r \leq m_{\alpha}, 1 \leq \alpha \leq \gamma\right\}$ is a basis for $\mathcal{Y}$. The set of algebraic multiplicities $\left\{m_{\alpha}+1\right\}$ is independent of the choice of basis for $\operatorname{ker} T_{\phi} T_{\phi^{-1}}$ and $\sum_{1}^{\gamma} m_{\alpha}+\gamma=\operatorname{dim} \mathcal{Y}$.

In what follows we take the collection $\left\{u_{\alpha}\right\}_{1}^{\gamma}$ so that for $j \geq \alpha \geq 1$ we have $m_{\alpha} \geq 1$ and for $\gamma \geq \alpha \geq j+1$ we have $m_{\alpha}=0$. Let $\mathcal{Z}$ be obtained from $\mathcal{Y}$ by replacing $\bigvee_{\alpha=1}^{\gamma}\left\{u_{\alpha}\right\}$ with the kernel of $T_{\phi}=\operatorname{ker}\left(T_{\phi} T_{\phi^{-1}}\right)^{*}$. Then we have the direct sum decomposition $H^{2}(\mathbb{T})=\mathcal{X} \dot{+} \mathcal{Z}$.

Moreover, $\operatorname{det}\left(\left.P_{\mathcal{Y}} X_{n}^{-1}\right|_{\mathcal{Y}}\right)=\operatorname{det}\left(\left.P_{\mathcal{Z}} X_{n}^{-1}\right|_{\mathcal{Z}}\right)$. So we can work with $\mathcal{Z}$ instead of $\mathcal{Y}$. The presence of the projection $P\left(T_{\bar{\phi}}\right)$ in the right-hand side of Proposition 1.1 makes this change desirable.

For Fredholm operators $T$ let $R(T)$ denote the regularizer of $T$ with respect to the trace ideal so that $T R(T)=1-P\left(T^{*}\right), R(T) T=1-P(T)$, with $P(T)$ denoting the orthogonal projection to the kernel of $T$. The Moore-Penrose inverse is such a regularizer.

Lemma 3.3. Suppose $\left\{B_{n}\right\}_{1}^{\infty}$ is a sequence of Fredholm operators which converges in the uniform norm to an operator $B$, and suppose also that $P\left(B_{n}\right)$ converges in the uniform norm to $P(B)$. Then $R\left(B_{n}\right)$ converges in the uniform norm to $R(B)$.

Proof. Recall that for any operator $A$ with closed range the Moore-Penrose inverse $A^{\dagger}$ has the form $R(A)=\left[A^{*} A+P(A)\right]^{-1} A^{*}$. Thus the assertion follows by continuity of the inverse map in the uniform topology.

\section{Corollary 3.1.}

$$
\lim _{n \rightarrow \infty} R\left(T_{\phi} S^{* n+1} R\left(T_{\phi}\right) S^{n+1}\right)=R\left(T_{\phi} T_{\phi^{-1}}\right) \quad \text { uniformly. }
$$

Proof. Since $T_{\phi}$ is injective $\operatorname{ker} T_{\phi} S^{* n+1} R\left(T_{\phi}\right) S^{n+1}=\operatorname{ker} S^{* n+1} R\left(T_{\phi}\right) S^{n+1}$. Also $S^{* n+1} R\left(T_{\phi}\right) S^{n+1}$ converges uniformly to $T_{\phi^{-1}}$. So the result follows by Lemmas 3.1 and 3.3 .

Now we will prove Lemma 1.1 which asserts that if $w_{1}, \ldots, w_{\gamma}$ is a basis for $\operatorname{ker} T_{\phi^{-1}}$, there is a basis $\left\{w_{\tau}^{(n)}\right\}$ in $\operatorname{ker}\left[S^{* n+1} R\left(T_{\phi}\right) S^{n+1}\right]$ so that $w_{\tau}^{(n)}$ converges to $w_{\tau}$ for $1 \leq \tau \leq \gamma$. 
Proof. Recall that by Lemma $3.1 P\left(S^{* n+1} R\left(T_{\phi}\right) S^{n+1}\right)$ converges to $P\left(T_{\phi^{-1}}\right)$. Hence we can take $w_{\alpha}^{(n)}=P\left(S^{* n+1} R\left(T_{\phi}\right) S^{n+1}\right) w_{\alpha}$.

Note that if $\left\{v_{\beta}^{(n)}\right\}_{1}^{\gamma}$ are the vectors obtained by applying the Gram-Schmidt pro-

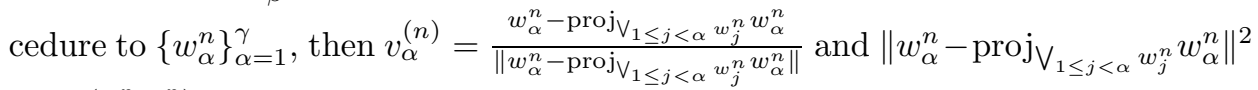
$=\frac{\operatorname{det}\left(w_{i}^{n}, w_{j}^{n}\right)_{1 \leq i, j \leq \alpha}}{\operatorname{det}\left(w_{i}^{n}, w_{j}^{n}\right)_{1 \leq i, j<\alpha}} \longrightarrow 1, \lim _{n \rightarrow \infty} v_{\alpha}^{(n)}=v_{\alpha}$, where the $v_{\alpha}$ are obtained by applying the Gram-Schmidt procedure to $w_{\alpha}$.

The action of $\left.P_{\mathcal{Z}} X_{n}^{-1}\right|_{\mathcal{Z}}$. We consider first the action of $P_{\mathcal{Z}} X_{n}^{-1}$ on the vectors $\left\{\left(T_{\phi} T_{\phi^{-1}}\right)^{r} u_{\alpha}\right.$. For $m_{\alpha} \geq r \geq 1$, define $z_{r \alpha}(n)=X_{n}^{-1}\left(T_{\phi} T_{\phi^{-1}}\right)^{r} u_{\alpha}$. Then $\left(T_{\phi} T_{\phi^{-1}}\right)^{r} u_{\alpha}=X_{n} z_{r \alpha}(n)$. Since $r \geq 1$, we have $P\left(T_{\bar{\phi}}\right) S^{n+1} z_{r \alpha}(n)=0$ so that

$$
\begin{aligned}
\left(T_{\phi} T_{\phi^{-1}}\right)^{r} u_{\alpha} & =T_{\phi} S^{* n+1} R\left(T_{\phi}\right) S^{n+1} z_{r \alpha}(n) \\
& =T_{\phi} S^{* n+1} R\left(T_{\phi}\right) S^{n+1}\left[1-P\left(S^{* n+1} R\left(T_{\phi}\right) S^{n+1}\right)\right] z_{r \alpha}(n)
\end{aligned}
$$

and

$$
R\left(T_{\phi} S^{* n+1} R\left(T_{\phi}\right) S^{n+1}\right)\left(T_{\phi} T_{\phi^{-1}}\right)^{r} u_{\alpha}=\left[1-P\left(S^{* n+1} R\left(T_{\phi} S^{n+1}\right)\right] z_{r \alpha}(n) .\right.
$$

By Lemma 3.1, $P\left(S^{* n+1} R\left(T_{\phi}\right) S^{n+1}\right)$ converges in the trace norm to $P\left(T_{\phi^{-1}}\right)=$ $P\left(T_{\phi} T_{\phi^{-1}}\right)$.

Therefore, by Corollary 3.1 it follows that $\left[1-P\left(S^{* n+1} R\left(T_{\phi}\right) S^{n+1}\right)\right] z_{r \alpha}(n) \quad$ converges to $\quad\left[1-P\left(T_{\phi} T_{\phi^{-1}}\right)\right]\left(T_{\phi} T_{\phi^{-1}}\right)^{r-1} u_{\alpha}$, and $\tilde{z}_{r \alpha}(n) \equiv X_{n}\left[1-P\left(S^{* n+1} R\left(T_{\phi}\right) S^{n+1}\right)\right] z_{r \alpha}(n)$ converges to the basis vector $\left(T_{\phi} T_{\phi^{-1}}\right)^{r} u_{\alpha}$. Note that $\mathcal{Z}=\bigvee_{1 \leq r \leq m_{\alpha}, 1 \leq \alpha \leq \gamma}\left\{\tilde{z}_{r \alpha}(n)\right\}+\operatorname{ker} T_{\bar{\phi}}$. Furthermore,

$$
\begin{aligned}
P_{\mathcal{Z}} X_{n}^{-1} \tilde{z}_{r \alpha}(n) & =P_{\mathcal{Z}}\left[1-P\left(S^{* n+1} R\left(T_{\phi}\right) S^{n+1}\right)\right] z_{r \alpha}(n) \\
& \longrightarrow P_{\mathcal{Z}}\left[1-P\left(T_{\phi} T_{\phi^{-1}}\right)\right]\left(T_{\phi} T_{\phi^{-1}}\right)^{r-1} u_{\alpha} .
\end{aligned}
$$

Next we consider $P_{\mathcal{Z}} X_{n}^{-1}$ acting on $\operatorname{ker} T_{\bar{\phi}}$. Let $y_{1}, \ldots, y_{\gamma}$ be an orthonormal basis for $\operatorname{ker} T_{\bar{\phi}}$. Set $z_{\alpha}(n)=X_{n}^{-1} y_{\alpha}$. Then $X_{n} z_{\alpha}(n)=y_{\alpha}$ so that

$$
S^{* n+1} R\left(T_{\phi}\right) S^{n+1} z_{\alpha}(n)=0 \quad \text { and } \quad\left(S^{n+1} z_{\alpha}(n), y_{\tau}\right)=\delta_{\alpha \tau} .
$$

Let $\left\{w_{\alpha}^{(n)}\right\}_{1}^{\gamma}$ be any basis for $\operatorname{ker}\left(S^{* n+1} T_{\phi} S^{n+1}\right)$. and suppose that $\left\{v_{\alpha}^{(n)}\right\}_{1}^{\gamma}$ denotes the associated orthonormal basis derived by the Gram-Schmidt procedure. Then, since $\left.z_{\alpha}(n)=\sum_{\alpha=1}^{\gamma}\left(z_{\alpha}(n), v_{\tau}^{(n)}\right) v_{\tau}^{(n)}\right)$, we have

$$
\delta_{\alpha \mu}=\left(S^{n+1} z_{\alpha}(n), y_{\mu}\right)=\sum_{\tau=1}^{\gamma}\left(S^{n+1} v_{\tau}^{(n)}, y_{\mu}\right)\left(z_{\alpha}(n), v_{\tau}^{(n)}\right)
$$

and it follows that

$$
\operatorname{det}\left(\left(z_{\alpha}(n), v_{\tau}^{(n)}\right)\right)=\frac{1}{\operatorname{det}\left(\left(S^{n+1} v_{\alpha}^{(n)}, y_{\tau}\right)\right)} .
$$

Let $C_{n}$ and $K_{n}$ denote the linear maps defined on $\mathcal{Z}$ so that for $\alpha=1,2, \ldots, \gamma$,

1) $C_{n}\left(y_{\alpha}\right)=\sum_{\ell=1}^{\gamma}\left(S^{* n+1} v_{\alpha}^{(n)}, y_{\ell}\right) y_{\ell}$,

2) $\quad C_{n}\left(T_{\phi} T_{\phi^{-1}}\right)^{r} u_{\alpha}=\tilde{z}_{r \alpha}(n)=\left(T_{\phi} T_{\phi^{-1}}\right)^{r} u_{\alpha}-d_{r \alpha}(n)$, where $d_{r \alpha}(n) \in \operatorname{ker} T_{\bar{\phi}}$,

3) $K_{n}\left(y_{\alpha}\right)=P_{\mathcal{Z}}\left(v_{\alpha}^{(n)}\right)$,

4) $K_{n}\left(T_{\phi} T_{\phi^{-1}}\right)^{r} u_{\alpha}=P_{\mathcal{Z}} R\left(T_{\phi} S^{* n+1} R_{\phi} S^{n+1}\right)\left(T_{\phi} T_{\phi^{-1}}\right)^{r} u_{\alpha}$ for $m_{\alpha} \geq r \geq 1$. 
Then

$$
K_{n}=P_{\mathcal{Z}} X_{n}^{-1} P_{\mathcal{Z}} C_{n}
$$

To check this, first consider the action on $y_{\alpha}$. We have

$$
\begin{aligned}
X_{n}^{-1} P_{\mathcal{Z}} C_{n}\left(y_{\alpha}\right) & =X_{n}^{-1}\left[\sum_{\ell=1}^{\gamma}\left(S^{n+1} v_{\alpha}^{(n)}, y_{\ell}\right) y_{\ell}\right]=\sum_{\ell=1}^{\gamma}\left(S^{n+1} v_{\alpha}^{(n)}, y_{\ell}\right) z_{\ell}(n) \\
& =\sum_{\ell=1}^{\gamma}\left(S^{n+1} v_{\alpha}^{(n)}, y_{\ell}\right)\left[\sum_{\tau=1}^{\gamma}\left(z_{\ell}(n), v_{\tau}^{(n)}\right) v_{\tau}^{(n)}\right] \\
& =\sum_{\tau=1}^{\gamma}\left[\sum_{\ell=1}^{\gamma}\left(S^{n+1} v_{\alpha}^{(n)}, y_{\ell}\right) \cdot\left(z_{\ell}(n), v_{\tau}^{(n)}\right)\right] v_{\tau}^{(n)} .
\end{aligned}
$$

After taking the transpose of equation (3.2), the inner sum becomes $\delta_{\alpha \tau}$ and therefore $P_{\mathcal{Z}} X_{n}^{-1} C_{n} y_{\alpha}=P_{\mathcal{Z}} v_{\alpha}^{(n)}$.

Next we consider the action on vectors $\left(T_{\phi} T_{\phi^{-1}}\right)^{r} u_{\alpha}$. It follows that

$$
\begin{aligned}
X_{n}^{-1} P_{\mathcal{Z}} C_{n}\left(T_{\phi} T_{\phi^{-1}}\right)^{r} u_{\alpha} & =\left[1-P\left(S^{* n+1} R\left(T_{\phi}\right) S^{n+1}\right)\right] z_{r_{\alpha}}(n) \\
& =R\left(T_{\phi} S^{* n+1} R\left(T_{\phi}\right) S^{n+1}\right)\left(T_{\phi} T_{\phi^{-1}}\right)^{r} u_{\alpha} ;
\end{aligned}
$$

thus $K_{n}=P_{\mathcal{Z}} X_{n}^{-1} P_{\mathcal{Z}} C_{n}$.

Now, since $\operatorname{det}\left(C_{n}\right)=\operatorname{det}\left(S^{n+1} v_{\alpha}^{(n)}, y_{\tau}\right)$, it follows that

$$
\operatorname{det}\left(\left.P_{\mathcal{Y}} X_{n}^{-1}\right|_{\mathcal{Y}}\right)=\operatorname{det}\left(\left.P_{\mathcal{Z}} X_{n}^{-1}\right|_{\mathcal{Z}}\right)=\frac{\operatorname{det}\left(K_{n}\right)}{\operatorname{det}\left(C_{n}\right)}=\frac{\operatorname{det}\left(K_{n}\right)}{\operatorname{det}\left(S^{n+1} v_{\alpha}^{(n)}, y_{\tau}\right)},
$$

and therefore,

$$
D_{n}\left(T_{\phi}\right)=\mathbf{G}(\phi)^{n+1} \frac{\left.\operatorname{det} P_{\mathcal{X}} X_{n \mid \mathcal{X}}\right)}{\operatorname{det}\left(K_{n}\right)} \cdot \operatorname{det}\left(S^{n+1} v_{\alpha}^{(n)}, y_{\tau}\right) \quad \text { provided } \quad D_{n}(\phi) \neq 0 .
$$

On the other hand, since $\operatorname{dim} \operatorname{ker}\left(S^{* n+1} R\left(T_{\phi}\right) S^{n+1}\right)=\gamma$ when $n \gg 0$, the operator $K_{n}$ is defined even when $X_{n}$ is singular. By Gram-Schmidt, if $w_{\alpha}^{(n)} \rightarrow w_{\alpha}$, then $v_{\alpha}^{(n)} \rightarrow v_{\alpha}$ where $\left\{v_{\alpha}\right\}_{1}^{\gamma}$ is derived from $\left\{w_{\alpha}\right\}_{1}^{\gamma}$ by the Gram-Schmidt procedure. In that case, $K_{n}$ converges to the operator $K$ defined by

$$
\begin{gathered}
K\left(y_{\alpha}\right)=P_{\mathcal{Z}} v_{\alpha}, \\
K\left(T_{\phi} T_{\phi^{-1}}\right)^{r} u_{\alpha}=P_{\mathcal{Z}}\left[1-P\left(T_{\phi} T_{\phi^{-1}}\right)\right]\left(T_{\phi} T_{\phi^{-1}}\right)^{r-1} u_{\alpha} \quad \text { for } \quad m_{\alpha} \geq r \geq 1 .
\end{gathered}
$$

Since $\mathcal{Z}$ is finite dimensional, $K_{n}$ will be invertible provided that $K$ is invertible.

Lemma 3.4. $\operatorname{det}(K)=(-1)^{\sum_{1}^{\gamma} m_{\alpha}} \operatorname{det}\left(b_{\alpha}, y_{\ell}\right)$ where $\left\{b_{\alpha}\right\}_{1}^{\gamma}$ is any set of $T_{\phi} T_{\phi^{-1}}$ maximal vectors for $\left\{v_{\alpha}\right\}_{1}^{\gamma}$. In particular, $K$ is invertible.

Proof. Let $L: \mathcal{Z} \rightarrow \mathcal{Y}$ be the linear map where for $\alpha=1,2, \ldots, \gamma, L y_{\alpha}=v_{\alpha}$ and $L\left(T_{\phi} T_{\phi^{-1}}\right)^{r} u_{\alpha}=\left[1-P\left(T_{\phi} T_{\phi^{-1}}\right)\right]\left(T_{\phi} T_{\phi^{-1}}\right)^{r-1} u_{\alpha}$, for $1 \leq r \leq m_{\alpha}$. Then $K=P_{\mathcal{Z}} L$. Hence, $\operatorname{det} K=\operatorname{det} P_{\mathcal{Z}} L=\operatorname{det} L P_{\mathcal{Z} \mid \mathcal{Y}}$.

Let

and let

$$
\mathcal{Y}_{1}=\bigvee_{\alpha=1}^{\gamma}\left\{u_{\alpha}\right\} \quad \text { and } \quad \mathcal{Y}_{2}=\bigvee_{\alpha=1}^{\gamma} \bigvee_{r=1}^{m_{\alpha}}\left\{\left(T_{\phi} T_{\phi}^{-1}\right)^{r} u_{\alpha}\right\}
$$

$$
\mathcal{Y}_{3}=\bigvee_{\alpha=1}^{\gamma}\left\{w_{\alpha}\right\} \quad \text { and } \quad \mathcal{Y}_{4}=\operatorname{ran}\left(1-P\left(T_{\phi} T_{\phi^{-1}}\right)\right) \mathcal{Y}
$$


Then

$$
\mathcal{Y}=\mathcal{Y}_{1}+\mathcal{Y}_{2}=\mathcal{Y}_{3}+\mathcal{Y}_{4}
$$

Note that $\mathcal{Y}_{4}=\operatorname{rp}\left[\left(T_{\phi} T_{\phi^{-1}}\right)^{*}\right] \mathcal{Y}$. Let $P\left(\mathcal{Y}_{3}\right)$ be the linear map on $\mathcal{Y}$ which acts as the projection onto $\mathcal{Y}_{3}$ parallel to $\mathcal{Y}_{4}$. Define the linear map $M: \mathcal{Y} \rightarrow \mathcal{Y}$ by setting

$$
M=\left(P\left(\mathcal{Y}_{3}\right) L P_{\mathcal{Z}_{\mathcal{Y}_{1}}}\right)^{-1} P\left(\mathcal{Y}_{3}\right)+\left(L P_{\mathcal{Z}_{\mathcal{Y}_{2}}}\right)^{-1}\left(1-P\left(\mathcal{Y}_{3}\right)\right)
$$

Computation shows that $\left.M L P_{\mathcal{Z}}\right|_{\mathcal{Y}}=I+N$ where $N \mathcal{Y}_{2}=0$, and $N: \mathcal{Y}_{1} \rightarrow$ $\mathcal{Y}_{2}$. Therefore, $\left.\operatorname{det} L P_{\mathcal{Z}}\right|_{\mathcal{Y}}=\operatorname{det} M^{-1}$. Further computation shows $M^{-1} u_{\alpha}=$ $\sum_{\rho=1}^{\gamma} a_{\alpha \rho} w_{\rho}$ where $a_{\alpha \rho}=\sum_{\tau=1}^{\gamma}\left(u_{\alpha}, y_{\tau}\right) \Gamma_{\tau \rho}$ with $\left(\Gamma_{\tau, \rho}\right)$ the matrix relative to $\left.\mid w_{\tau}\right\}_{1}^{\gamma}$ for the map defined by $\Gamma\left(w_{\tau}\right)=v_{\tau}$, and we also have

$$
M^{-1}\left(T_{\phi} T_{\phi^{-1}}\right)^{r} u_{\alpha}=\left[1-P\left(T_{\phi} T_{\phi^{-1}}\right)\right]\left(T_{\phi} T_{\phi^{-1}}\right)^{r-1} u_{\alpha} .
$$

Recall that the $\left\{u_{\alpha}\right\}_{1}^{\gamma}$ have been so ordered that $1 \leq m_{\alpha}$ for $1 \leq \alpha \leq j$, and $m_{\alpha}=0$ for $j+1 \leq \alpha \leq \gamma$. Thus, if $X=T_{\phi} T_{\phi^{-1}}$, then $M^{-1}$ has the following matrix representation:

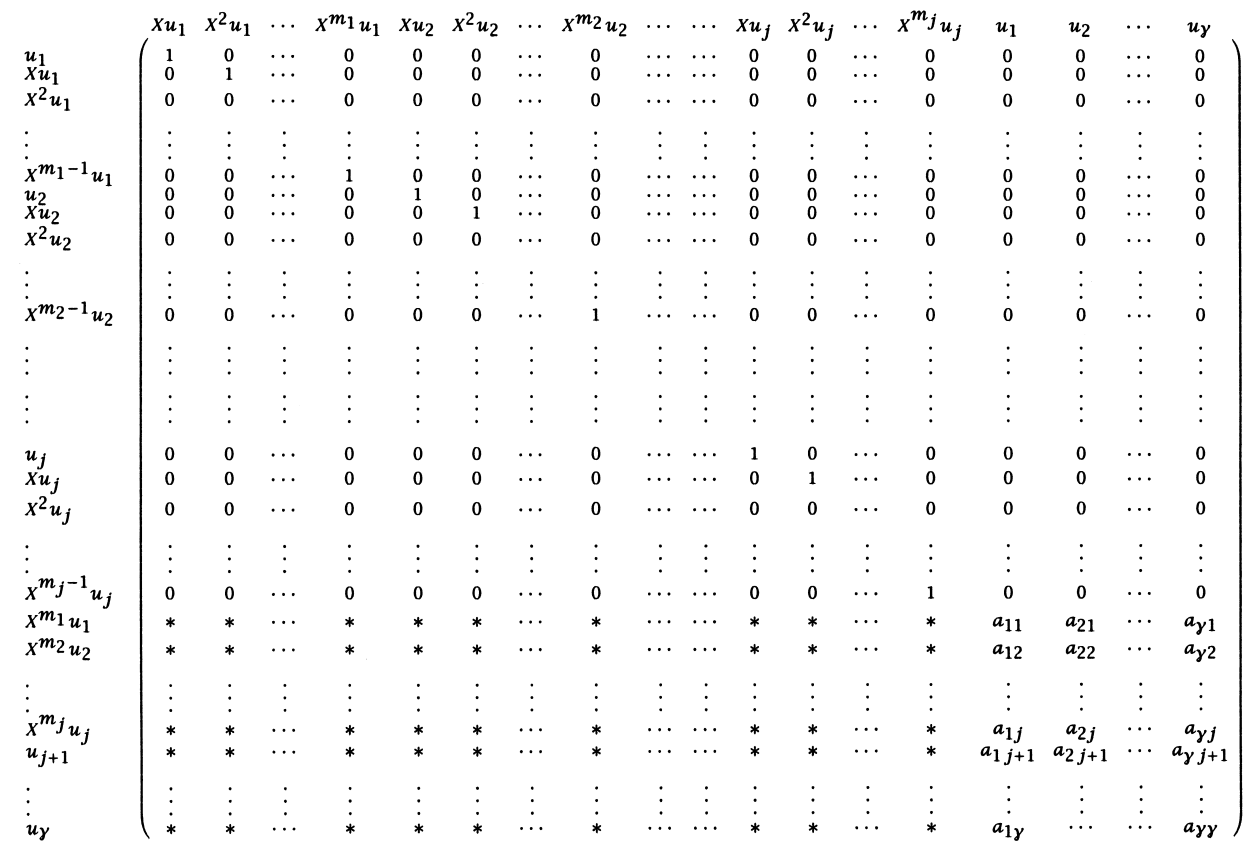

Now $\operatorname{det} \Gamma=\frac{1}{\left\|\wedge w_{\alpha}\right\|}$. Therefore by ii) of Proposition 2.1 we have $\operatorname{det} K=\operatorname{det} M^{-1}=(-1)^{\sum_{\alpha=1}^{\gamma} m_{\alpha}} \operatorname{det}\left(u_{\alpha}, y_{\tau}\right) \cdot \operatorname{det}(\Gamma)=(-1)^{\sum_{\alpha=1}={ }^{\gamma} m_{\alpha}} \operatorname{det}\left(b_{\alpha}, y_{\tau}\right)$, where the $\left\{b_{\alpha}\right\}_{1}^{\gamma}$ are maximal vectors for $\left\{v_{\alpha}\right\}_{1}^{\gamma}$.

Now, since $K_{n}$ is invertible, and $D_{n}\left(T_{\phi}\right)=0$ if and only if $\operatorname{det}\left(S^{n+1} v_{\alpha}^{(n)}, y_{\tau}\right)=0$, we have shown that for all $n \gg 0$,

$$
D_{n}(\phi)=G(\phi)^{n+1} \frac{\operatorname{det}\left(P_{\mathcal{X}} X_{n \mid \mathcal{X}}\right)}{\operatorname{det}\left(K_{n}\right)} \cdot \operatorname{det}\left(S^{n+1} v_{\alpha}^{(n)}, y_{\tau}\right) \text {. }
$$


Let $\Gamma^{(n)}$ be the linear map on $\operatorname{ker}\left(S^{* n+1} R\left(T_{\phi}\right) S^{n+1}\right)$ so that $\Gamma^{(n)}\left(w_{\alpha}^{(n)}\right)=v_{\alpha}^{(n)}$. Then $\operatorname{det}\left(\Gamma^{(n)}\right) \rightarrow \operatorname{det}(\Gamma)=\frac{1}{\left\|\wedge w_{\alpha}\right\|}$, where as above $\Gamma\left(w_{\alpha}\right)=v_{\alpha}$.

Let $\left\{t_{\tau}\right\}_{1}^{\gamma}$ be any basis for ker $T_{\phi}$. Then upon substitution into (3.3), Proposition 2.1 gives

$D_{n}(\phi)=\mathbf{G}(\phi)^{n+1} \cdot \operatorname{det}\left(S^{n+1} w_{\alpha}^{(n)}, t_{\tau}\right) \cdot\left[(-1)^{\sum_{1}^{\gamma} m_{\alpha}} \frac{\left.\prod_{\lambda \in \sigma\left(T_{\phi} T_{\phi}-1\right.}\right) \backslash\{0\}}{\lambda}\right] \cdot[1+o(1)]$,

where the eigenvalues $\lambda$ are repeated according to their multiplicity. Again the equality $\left.\operatorname{det} T_{\phi} T_{\phi^{-1}}\right|_{\mathcal{X}}=\prod_{\lambda \in \sigma\left(T_{\phi} T_{\phi^{-1}}\right) \backslash\{0\}} \lambda$ follows from the Riesz-Schauder decomposition and the well-known result of V.B. Lidskii. This completes the proof of part i) of Theorem 1.1.

\section{Proofs of Theorem 1.2 and Theorem 1.6}

Assertion a) of Theorem 1.2 is contained in Proposition 4.1 below. Assertion c) follows from b) while b) is a special case of Proposition 4.3 with $\Theta_{1}=z^{\gamma}$ and $\Theta_{2}=1$.

Let $\phi=\Theta_{1} f \cdot \bar{\Theta}_{2} \bar{g}$ with $\Theta_{1}$ and $\Theta_{2}$ coprime finite Blaschke products, and let $f$ and $g$ be outer functions in $K_{2,2}^{\frac{1}{2}, \frac{1}{2}} \cap \mathcal{W}$, the intersection of the Krein algebra $\left\{a \in \mathcal{L}(\mathbb{T})^{\infty}: \sum_{n \in Z}(|n|+1)\left|a_{n}\right|^{2}<\infty\right.$ and the Wiener algebra, $\mathcal{W}$. Note that if $a \in K_{2,2}^{\frac{1}{2}, \frac{1}{2}} \cap \mathcal{W}$ and has no zeros on $\mathbb{T}$, then $a^{-1} \in K_{2,2}^{\frac{1}{2}, \frac{1}{2}} \cap \mathcal{W}$. In this case the Hankel operators $H(a)$ and $H\left(a^{-1}\right)$ are in Hilbert-Schmidt class; cf. [12]. Let $\gamma \equiv \operatorname{wind}(\phi, 0)=\operatorname{deg} \Theta_{1}-\operatorname{deg} \Theta_{2}>0$. Note that $\operatorname{ker} T_{\bar{\phi}}=T_{\frac{1}{g}}\left[\operatorname{ker} T_{\bar{\Theta}_{1} \Theta_{2}}\right]$, $\operatorname{ker} T_{\phi^{-1}}=T_{f} \operatorname{ker} T_{\bar{\Theta}_{1} \Theta_{2}}$ and $\operatorname{ker} T_{\bar{\Theta}_{1} \Theta_{2}}=T_{\bar{\Theta}_{2}}\left[K_{\Theta_{1}} \cap K_{\Theta_{2}}^{\perp}\right]$.

Lemma 4.1. For $h \in \mathcal{L}^{\infty}(\mathbb{T})$ and the multiplication operator $M_{h}$ on $\mathcal{L}^{2}(\mathbb{T})$

$$
\begin{array}{cc}
K_{\Theta_{1}} \cap K_{\Theta_{2}}^{\perp} \stackrel{P\left(T_{\bar{\Theta}_{1}}\right) \wedge \operatorname{rp}\left(T_{\Theta_{2}}\right) M_{h}}{\longrightarrow} K_{\Theta_{1}} \cap K_{\Theta_{2}}^{\perp} \\
T_{\bar{\Theta}_{2}} \downarrow \\
\operatorname{ker} T_{\bar{\Theta}_{1} \Theta_{2}} \stackrel{P\left(T_{\bar{\Theta}_{1} \Theta_{2}}\right) M_{h}}{\longrightarrow} \quad \operatorname{ker} T_{\bar{\Theta}_{1} \Theta_{2}}
\end{array}
$$

Proof. The result is a consequence of the following two facts:

1) $P\left(T_{\bar{\Theta}_{1} \Theta_{2}}\right)=T_{\bar{\Theta}_{2}} P\left(T_{\bar{\Theta}_{1}}\right) \wedge \operatorname{rp}\left(T_{\Theta_{2}}\right) T_{\Theta_{2}}$,

2) $P\left(T_{\bar{\Theta}_{1}}\right) \wedge \operatorname{rp}\left(T_{\Theta_{2}}\right) \cdot M_{\Theta_{2}} P=P\left(T_{\bar{\Theta}_{1}}\right) \wedge \operatorname{rp}\left(T_{\Theta_{2}}\right) \cdot M_{\Theta_{2}}$.

As a consequence of the lemma we see that

$$
\begin{aligned}
& \left.\operatorname{det} P\left(T_{\bar{\Theta}_{1} \Theta_{2}}\right) M_{h}\right|_{\text {ker } T_{\bar{\Theta}_{1} \Theta_{2}}}=\operatorname{det} P\left(T_{\bar{\Theta}_{1}}\right) \wedge \operatorname{rp}\left(T_{\Theta_{2}}\right) M_{h_{K_{\Theta_{1}} \cap K \bar{\Theta}_{2}}}, \\
& \operatorname{dim} \operatorname{ker} P\left(T_{\bar{\Theta}_{1} \Theta_{2}}\right) M_{\left.\right|_{\operatorname{ker} T_{\bar{\Theta}_{1} \Theta_{2}}}}=\operatorname{dim} \operatorname{ker} P\left(T_{\bar{\Theta}_{1}}\right) \wedge \operatorname{rp}\left(T_{\Theta_{2}}\right) M_{\left.\right|_{K_{\Theta_{1}} \cap K_{\Theta_{2}}}} .
\end{aligned}
$$

Fix orthonormal bases $\left\{w_{\alpha}\right\}_{1}^{\gamma}$ and $\left\{y_{\alpha}\right\}_{1}^{\gamma}$ of $\operatorname{ker} T_{\phi^{-1}}$ and $\operatorname{ker} T_{\bar{\phi}}$. Pick $\left\{s_{\alpha}\right\}$ to be orthonormal eigenvectors of $P\left(T_{\bar{\Theta}_{1} \Theta_{2}}\right) T_{|f|^{2} \mid \operatorname{ker} T_{\bar{\Theta}_{1} \Theta_{2}}}$ so that

$$
P\left(T_{\bar{\Theta}_{1} \Theta_{2}}\right) T_{|f|^{2} \mid \operatorname{ker} T_{\bar{\Theta}_{1} \Theta_{2}}} s_{\alpha}=\mu_{\alpha} s_{\alpha}
$$


Then $\left\{\frac{1}{\sqrt{\mu_{\beta}}} T_{f} s_{\tau}\right\}_{1}^{\gamma}$ is a complete orthonormal set in $\operatorname{ker} T_{\phi^{-1}}$. Similarly, pick $\left\{r_{\alpha}\right\}_{1}^{\gamma}$ to be orthonormal eigenvectors of $P\left(T_{\bar{\Theta}_{1} \Theta_{2}}\right) T_{\left|\frac{1}{g}\right|^{2} \mid \operatorname{ker} T_{\bar{\Theta}_{1} \Theta_{2}}}$ so that

$$
P\left(T_{\bar{\Theta}_{1} \Theta_{2}}\right) T_{\left|\frac{1}{g}\right|^{2} \mid \operatorname{ker} T_{\bar{\Theta}_{1} \Theta_{2}}} r_{\alpha}=\lambda_{\alpha} r_{\alpha}
$$

Then $\left\{\frac{1}{\sqrt{\lambda_{\beta}}} T_{\frac{1}{g}} r_{\beta}\right\}_{1}^{\gamma}$ is a complete orthonormal set in $\operatorname{ker} T_{\bar{\phi}}$. Let $W: \operatorname{ker} P\left(T_{\bar{\Theta}_{1} \Theta_{2}}\right) \rightarrow$ $\operatorname{ker} P\left(T_{\bar{\Theta}_{1} \Theta_{2}}\right)$ be the unitary map so that $W r_{\alpha}=s_{\alpha}$. Similarly, let $V: \operatorname{ker} T_{\phi^{-1}} \rightarrow$ $\operatorname{ker} T_{\phi^{-1}}$ be the unitary map such that $V T_{f} \frac{s_{\beta}}{\sqrt{\mu_{\beta}}}=w_{\beta}$ and let $U: \operatorname{ker} T_{\bar{\phi}} \rightarrow \operatorname{ker} T_{\bar{\phi}}$ be the unitary map so that $U T_{\frac{1}{g}} \frac{r_{\beta}}{\sqrt{\lambda} \beta}=y_{\beta}$.

Proposition 4.1. a) $\#\left\{\alpha: m_{\alpha} \neq 0\right\}=\left.\operatorname{dim} \operatorname{ker} P\left(T_{\bar{\Theta}_{1}}\right) \wedge \operatorname{rp}\left(T_{\Theta_{2}}\right) M_{\frac{f}{g}}\right|_{K_{\Theta_{1}} \cap K_{\Theta_{2}}^{\perp}}=$ $\operatorname{dim} \operatorname{ker} T_{\phi} T_{\phi^{-1}}$. In particular, if $\phi$ is unimodular, then $m_{\alpha}=0$ for all $\alpha$.

b) $\sum_{1}^{\gamma} m_{\alpha}=\operatorname{dim} \operatorname{root}$ space $\left[Q_{\gamma-1}\left(I-H_{\psi} H_{\frac{1}{\psi}} \mid \operatorname{ran} S^{\gamma}\right]\right.$,

Proof. a) Since $T_{\phi^{-1}}$ is surjective,

$$
j=\text { cardinality }\left\{\alpha: m_{\alpha} \neq 0\right\}=\operatorname{dim}\left[\operatorname{ran} T_{\phi} \cap \operatorname{ker} T_{\phi^{-1}}\right] .
$$

Now we may pick the basis $\left\{w_{\alpha}\right\}_{1}^{\gamma}$ so that $w_{1}, \ldots, w_{j}$ is a basis for $\left[\operatorname{ran} T_{\phi} \cap \operatorname{ker} T_{\phi^{-1}}\right]$ and then $w_{j+1}, \ldots, w_{\gamma}$ is a basis for $\left[\operatorname{ker} T_{\phi^{-1}} \ominus \operatorname{ran} T_{\phi} \cap \operatorname{ker} T_{\phi^{-1}}\right]$. Since the inner product $\left(w_{\alpha}, y_{\beta}\right)=\left(u_{\alpha}, y_{\beta}\right)$ for $j+1 \leq \alpha \leq \gamma$ and $\left(w_{\alpha}, y_{\beta}\right)=0$ for $1 \leq \alpha \leq j$, it follows that

$$
\begin{gathered}
\gamma-j=\#\left\{\alpha: m_{\alpha}=0\right\}=\text { column rank }\left(\left(w_{\alpha}, y_{\beta}\right)\right)=\text { column } \operatorname{rank}\left(\left(T_{f} s_{\alpha}, T_{\frac{1}{g}} r_{\beta}\right)\right) \\
=\text { column rank }\left(\left(T_{f} r_{\alpha}, T_{\frac{1}{g}} r_{\beta}\right)\right)=\left.\operatorname{dim} \operatorname{ran} P\left(T_{\bar{\Theta}_{1} \Theta_{2}}\right) T_{\frac{f}{g}}\right|_{\operatorname{ker} P\left(T_{\bar{\Theta}_{1} \Theta_{2}}\right)} \\
=\gamma-\left.\operatorname{dim} \operatorname{ker} P\left(T_{\bar{\Theta}_{1} \Theta_{2}}\right) T_{\frac{f}{g}}\right|_{\operatorname{ker} P\left(T_{\bar{\Theta}_{1} \Theta_{2}}\right)} \\
=\gamma-\left.\operatorname{dim} \operatorname{ker} P\left(T_{\bar{\Theta}_{1}}\right) \wedge \operatorname{rp}\left(T_{\Theta_{2}}\right) M_{\frac{f}{g}}\right|_{K_{\Theta_{1} \cap K_{\Theta_{2}}}} .
\end{gathered}
$$

If $\phi$ is unimodular, then $f=c \cdot \frac{1}{g}$ for some $c \in C^{*}$, and then $j=0$.

Proof. b) With $\phi=f \bar{g} z^{\gamma}$, and $\langle$,$\rangle denoting multiplicative commutator we have$

$$
T_{\phi} T_{\phi^{-1}}=T_{f \bar{g}} Q_{\gamma-1} T_{\frac{1}{f \bar{g}}}=T_{f}\left\langle T_{\frac{1}{f}}, T_{\bar{g}}\right\rangle T_{\bar{g}} Q_{\gamma-1} T_{\frac{1}{\bar{g}}} T_{\frac{1}{f}} .
$$

Therefore, $T_{\phi} T_{\phi^{-1}}$ is similar to

$$
T_{\frac{1}{\bar{g}}}\left\langle T_{\frac{1}{f}}, T_{\bar{g}}\right\rangle T_{\bar{g}} Q_{\gamma-1}=T_{\frac{1}{f \bar{g}}} T_{f \bar{g}} Q_{\gamma-1}=\left[1-H_{\frac{1}{f \bar{g}}} H_{\tilde{f} \bar{g}}\right] Q_{\gamma-1} .
$$

This last operator has the block matrix form $\left(\begin{array}{cc}M & 0 \\ L & 0\end{array}\right)$ relative to the decomposition $H^{2}(\mathbb{T})=\operatorname{ran} S^{\gamma} \oplus \operatorname{ker} S^{* \gamma}$. Since $H_{\frac{1}{f \bar{g}}} H_{\tilde{f} \tilde{g}}$ is trace class, we have for sufficiently 
small $\delta>0$

$$
\begin{gathered}
\gamma+\sum_{1}^{\gamma} m_{\alpha}=\operatorname{dim} \operatorname{root} \text { space } T_{\phi} T_{\phi^{-1}}=\operatorname{dim} \operatorname{root} \operatorname{space}\left(\begin{array}{cc}
M & 0 \\
L & 0
\end{array}\right) \\
=\operatorname{tr} \frac{1}{2 \pi i} \int_{|z|=\delta}\left(z\left(\begin{array}{cc}
1 & 0 \\
0 & 1
\end{array}\right)-\left(\begin{array}{cc}
M & 0 \\
L & 0
\end{array}\right)\right)^{-1} d z \\
=\operatorname{tr} \frac{1}{2 \pi i} \int_{|z|=\delta}\left(\begin{array}{cc}
(z-M)^{-1} & 0 \\
\frac{L}{z}(z-M)^{-1} & \frac{1}{z}
\end{array}\right) d z \\
=\frac{1}{2 \pi i} \int_{|z|=\delta} \operatorname{tr}\left(\begin{array}{cc}
(z-M)^{-1}-(z-1)^{-1} & 0 \\
\frac{L}{z}(z-M)^{-1} & \frac{1}{z}
\end{array}\right) d z \\
=\gamma+\operatorname{tr} \frac{1}{2 \pi i} \int_{\mid z=\delta}(z-M)^{-1} d z=\gamma+\operatorname{dim} \operatorname{root} \operatorname{space}\left[Q_{\gamma-1}\left(I-H_{\psi} H_{\frac{1}{\psi} \mid \operatorname{ran} S^{\gamma}}\right)\right]
\end{gathered}
$$

Proposition 4.2. Suppose that $m_{\alpha}=0, \quad \forall \alpha$. Then

i) $\quad \sigma_{1, T_{\phi} T_{\phi^{-1}}}=\frac{\prod_{\lambda \in \sigma\left(T_{\phi} T_{\phi}-1\right) \backslash\{0\}} \lambda}{\operatorname{det}\left(v_{\alpha}, y_{\tau}\right)} \bigwedge v_{\alpha} \otimes\left(\bigwedge y_{\tau}+\operatorname{ran} T_{\phi}\right)^{*}$

where $\left\{v_{\alpha}\right\}_{1}^{\gamma}$ and $\left\{y_{\tau}\right\}_{1}^{\gamma}$ are respectively orthonormal bases for $\operatorname{ker} T_{\phi^{-1}}$ and $\operatorname{ker} T_{\bar{\phi}}$. In particular,

$$
\left\|\sigma_{1, T_{\phi} T_{\phi^{-1}}}\right\|=\left|\frac{\prod_{\lambda \in \sigma\left(T_{\phi} T_{\phi^{-1}}\right) \backslash\{0\}} \lambda}{\chi\left(P\left(T_{\phi^{-1}}\right), P\left(T_{\bar{\phi}}\right)\right)}\right| .
$$

ii) $\prod_{\lambda \in \sigma\left(T_{\phi} T_{\phi^{-1}}\right) \backslash\{0\}} \lambda=\chi^{2}\left(P\left(T_{\bar{\Theta}_{1}}\right), P\left(T_{\bar{\Theta}_{2}}\right)\right)$

$\times \prod_{|x|<1} c_{x}\left(\frac{f}{\bar{g}}, \frac{\Theta_{2}}{\Theta_{1}}\right) \cdot \operatorname{det}\left(P\left(T_{\bar{\Theta}_{1}}\right) \wedge \operatorname{rp}\left(T_{\Theta_{2}}\right) \cdot T_{\frac{f}{\bar{g}} \mid K_{\Theta_{1}} \cap K_{\Theta_{2}}^{\perp}}\right) \times \operatorname{det}_{*} \partial\left\{T_{\bar{g}}+\mathcal{L}^{1}, T_{f}+\mathcal{L}^{1}\right\}$.

iii) With $e^{i \rho}=\operatorname{det} U^{*} \operatorname{det} V \operatorname{det} W$,

$$
\begin{aligned}
& \operatorname{det}\left(v_{j}, y_{k}\right)= \\
& \frac{\operatorname{det}\left(\left.P\left(T_{\bar{\Theta}_{1}}\right) \wedge \operatorname{rp}\left(T_{\Theta_{2}}\right) \cdot T_{\frac{f}{\bar{g}}}\right|_{K_{\Theta_{1} \cap K_{\Theta_{2}}}}\right) e^{i \rho}}{\operatorname{det}\left(\left.P\left(T_{\bar{\Theta}_{1}}\right) \wedge \operatorname{rp}\left(T_{\Theta_{2}}\right) \cdot T_{|f|^{2}}\right|_{K_{\Theta_{1} \cap K_{\Theta_{2}}}}\right)^{\frac{1}{2}} \cdot \operatorname{det}\left(P\left(T_{\bar{\Theta}_{1}}\right) \wedge \operatorname{rp}\left(T_{\Theta_{2}}\right) \cdot T_{\left|\frac{1}{g}\right|^{2} \mid K_{\Theta_{1}} \cap K_{\Theta_{2}}^{\perp}}\right)^{\frac{1}{2}}} .
\end{aligned}
$$

iv)

$$
\begin{gathered}
\frac{\prod_{\lambda \in \sigma\left(T_{\phi} T_{\phi}-1\right) \backslash\{0\}} \lambda}{\operatorname{det}\left(v_{j}, y_{k}\right)}=\chi^{2}\left(P\left(T_{\bar{\Theta}_{1}}\right), P\left(T_{\bar{\Theta}_{2}}\right)\right) \cdot \prod_{|x|<1} c_{x}\left(\frac{f}{\bar{g}}, \frac{\Theta_{2}}{\Theta_{1}}\right) \\
\cdot \operatorname{det}_{*} \partial\left\{T_{\bar{g}}+\mathcal{L}^{1}, T_{f}+\mathcal{L}^{1}\right\} e^{-i \rho} \operatorname{det}\left(P\left(T_{\bar{\Theta}_{1}}\right) \wedge \operatorname{rp}\left(T_{\Theta_{2}}\right) \cdot T_{|f|^{2} \mid K_{\Theta_{1}} \cap K_{\Theta_{2}}}\right)^{\frac{1}{2}} \\
\cdot \operatorname{det}\left(P\left(T_{\bar{\Theta}_{1}}\right) \wedge \operatorname{rp}\left(T_{\Theta_{2}}\right) \cdot T_{\left|\frac{1}{g}\right|^{2} \mid K_{\Theta_{1}} \cap K_{\Theta_{2}}}\right)^{\frac{1}{2}} .
\end{gathered}
$$

In particular, if $\Theta_{1}=z^{\gamma}, \Theta_{2}=1$, then

$$
E(\psi) G\left(\frac{\bar{g}}{f}\right)^{\gamma}=\frac{\prod_{\lambda \in \sigma\left(T_{\phi} T_{\phi}-1\right) \backslash\{0\}} \lambda}{D_{\gamma-1}\left(T_{\frac{f}{g}}\right)} .
$$


v) Suppose $\left\{x_{\alpha}\right\}_{1}^{\gamma}$ is an orthonormal basis for $\operatorname{ker} T_{\Theta_{1} \bar{\Theta}_{2}}$. Let $\left\{w_{\alpha}\right\}_{1}^{\gamma}$ be obtained from the vectors $\left\{T_{f} x_{\alpha}\right\}_{1}^{\gamma}$ via the Gram-Schmidt orthogonalization procedure. Similarly, let $\left\{y_{\alpha}\right\}_{1}^{\gamma}$ be the result of orthogonalizing the vectors $\left\{T_{\frac{1}{g}} x_{\alpha}\right\}_{1}^{\gamma}$. Then, with this choice of bases for $\operatorname{ker} T_{\phi^{-1}}$ and $\operatorname{ker} T_{\bar{\phi}}$ respectively, statements iii) and iv) hold with $\rho=0$.

Proof. Part i) follows immediately from Proposition 2.1 by taking $X=T_{\phi} T_{\phi}-1$. To prove ii) recall that since $T_{\phi}$ is injective, $T_{\phi^{-1}}$ is surjective and so $\operatorname{ran} T_{\phi} T_{\phi^{-1}}=$ $\operatorname{ran} T_{\phi}$. Consequently, $\mathcal{X}=\operatorname{ran} T_{\phi}$ and so

$$
\prod_{\lambda \in \sigma\left(T_{\phi} T_{\phi^{-1}}\right) \backslash\{0\}} \lambda=\operatorname{det}\left(\left.T_{\phi} T_{\phi^{-1}}\right|_{\mathcal{X}}\right)=\operatorname{det}\left(\left.T_{\phi} T_{\phi^{-1}}\right|_{\operatorname{ran} T_{\phi}}\right)=\operatorname{det}\left(T_{\phi^{-1}} T_{\phi}\right) .
$$

Since $\phi^{-1}=\Theta_{2} \bar{\Theta}_{1} \frac{1}{f} \frac{1}{\bar{g}}$, assertion ii) is obtained by the arguments given in the proofs of Theorems 3.6 and Theorem 3.7 of [17].

To prove ii) note that $u_{j}=w_{j}$ for $j=1, \ldots, \gamma$.

Recall that $\left\{s_{\alpha}\right\}$ are orthonormal eigenvectors of $P\left(T_{\bar{\Theta}_{1} \Theta_{2}}\right) T_{|f|^{2} \mid \operatorname{ker} T_{\bar{\Theta}_{1} \Theta 2}}$ so that

$$
P\left(T_{\bar{\Theta}_{1} \Theta_{2}}\right) T_{|f|^{2} \mid \operatorname{ker} T_{\bar{\Theta}_{1} \Theta_{2}}} s_{\alpha}=\mu_{\alpha} s_{\alpha},
$$

and $\left\{\frac{1}{\sqrt{\mu_{\beta}}} T_{f} s_{\tau}\right\}$ is a complete orthonormal set in $\operatorname{ker} T_{\phi^{-1}}$. Also, the $\left\{r_{\alpha}\right\}$ are an orthonormal set of eigenvectors of $P\left(T_{\bar{\Theta}_{1} \Theta_{2}}\right) T_{\left|\frac{1}{g}\right|^{2} \mid \operatorname{ker} T_{\bar{\Theta}_{1} \Theta_{2}}}$ so that

$$
P\left(T_{\bar{\Theta}_{1} \Theta_{2}}\right) T_{\left|\frac{1}{g}\right|^{2} \mid \operatorname{ker} T_{\bar{\Theta}_{1} \Theta_{2}}} r_{\alpha}=\lambda_{\alpha} r_{\alpha}
$$

and $\left\{\frac{1}{\sqrt{\lambda_{\beta}}} T_{\frac{1}{g}} r_{\tau}\right\}$ is a complete orthonormal set in $\operatorname{ker} T_{\bar{\phi}}$. Let $W: \operatorname{ker} P\left(T_{\bar{\Theta}_{1} \Theta_{2}}\right) \rightarrow$ $\operatorname{ker} P\left(T_{\bar{\Theta}_{1} \Theta_{2}}\right)$ be the unitary map so that $W r_{\alpha}=s_{\alpha}$ and $V: \operatorname{ker} T_{\phi^{-1}} \rightarrow \operatorname{ker} T_{\phi^{-1}}$ is the unitary map such that $V T_{f} \frac{s_{\tau}}{\sqrt{\mu}_{\tau}}=w_{\tau}$ while $U: \operatorname{ker} T_{\bar{\phi}} \rightarrow \operatorname{ker} T_{\bar{\phi}}$ is the unitary map so that $U T_{\frac{1}{g}} \frac{r_{\tau}}{\sqrt{\lambda}_{\tau}}=y_{\tau}$.

Then

$$
\begin{gathered}
\operatorname{det}\left(w_{j}, y_{k}\right)=\operatorname{det}\left(V T_{f} W \frac{r_{j}}{\sqrt{\mu}_{j}}, U T_{\frac{1}{g}} \frac{r_{k}}{\sqrt{\lambda_{k}}}\right)=\operatorname{det} U^{*} \operatorname{det} V \operatorname{det} W \\
\times \frac{\operatorname{det}\left(\left.P\left(T_{\bar{\Theta}_{1}}\right) \wedge \operatorname{rp}\left(T_{\Theta_{2}}\right) \cdot T_{\frac{f}{\bar{g}}}\right|_{K_{\Theta_{1}} \cap K_{\Theta_{2}}}\right)}{\operatorname{det}\left(P\left(T_{\bar{\Theta}_{1}}\right) \wedge \operatorname{rp}\left(T_{\Theta_{2}}\right) \cdot T_{|f|^{2} \mid K_{\Theta_{1}} \cap K_{\Theta_{2}}^{\perp}}\right)^{\frac{1}{2}} \cdot \operatorname{det}\left(P\left(T_{\bar{\Theta}_{1}}\right) \wedge \operatorname{rp}\left(T_{\Theta_{2}}\right) \cdot T_{\left|\frac{1}{g}\right|^{2} \mid K_{\Theta_{1}} \cap K_{\Theta_{2}}^{\perp}}\right)^{\frac{1}{2}}} .
\end{gathered}
$$

Finally, set $\operatorname{det} U^{*} \operatorname{det} V \operatorname{det} W=e^{i \rho}$.

Combining ii) and iii) we arrive at iv).

Proof of v). It suffices to prove iii) with $\rho=0$. We have

$$
u_{j}=w_{j}=\frac{\left[T_{f} x_{j}-\operatorname{Proj}_{\bigvee_{1 \leq i<j} T_{f} x_{i}} T_{f} x_{j}\right]}{\left\|T_{f} x_{j}-\operatorname{Proj}_{\bigwedge_{1 \leq i<j} T_{f} x_{i}} T_{f} x_{j}\right\|}
$$

Therefore,

$$
\bigwedge_{j=1}^{\gamma} u_{j}=\frac{\bigwedge_{j=1}^{\gamma} T_{f} x_{j}}{\prod_{j=1}^{\gamma}\left\|T_{f} x_{j}-\operatorname{Proj}_{\bigvee_{1 \leq i \leq j} T_{f} x_{i}}\right\|}
$$


Since $\left\|\wedge u_{j}\right\|=1$,

$$
\prod_{j=1}^{\gamma}\left\|T_{f} x_{j}-\operatorname{Proj}_{\bigvee_{1 \leq i<j}} T_{f} e_{i} T_{f} x_{j}\right\|^{2}=\left\|\bigwedge_{j=1}^{\gamma} T_{f} x_{\alpha}\right\|^{2}=\operatorname{det}\left(T_{f} x_{\alpha}, T_{f} x_{\tau}\right)_{1 \leq \alpha, \tau \leq \gamma}
$$

Consequently,

$$
\bigwedge_{j=1}^{\gamma} u_{j}=\frac{\bigwedge_{j=1}^{\gamma} T_{f} x_{j}}{\operatorname{det}\left(P\left(T_{\bar{\Theta}_{1}}\right) \wedge \operatorname{rp}\left(T_{\Theta_{2}}\right) \cdot T_{|f|^{2} \mid K_{\Theta_{1}} \cap K_{\Theta_{2}}^{\perp}}\right)^{\frac{1}{2}}},
$$

and similarly,

$$
\bigwedge_{j=1}^{\gamma} y_{j}=\frac{\bigwedge_{j=1}^{\gamma} T_{\frac{1}{g}} x_{j}}{\operatorname{det}\left(P\left(T_{\bar{\Theta}_{1}}\right) \wedge \operatorname{rp}\left(T_{\Theta_{2}}\right) \cdot T_{\left|\frac{1}{g}\right|^{2} \mid K_{\Theta_{1}} \cap K_{\Theta_{2}}^{\perp}}\right)^{\frac{1}{2}}}
$$

Therefore,

$$
\begin{aligned}
& \operatorname{det}\left(u_{j}, y_{i}\right)=\left(\bigwedge_{j=1}^{\gamma} u_{j}, \bigwedge_{=1}^{\gamma} y_{j}\right)
\end{aligned}
$$

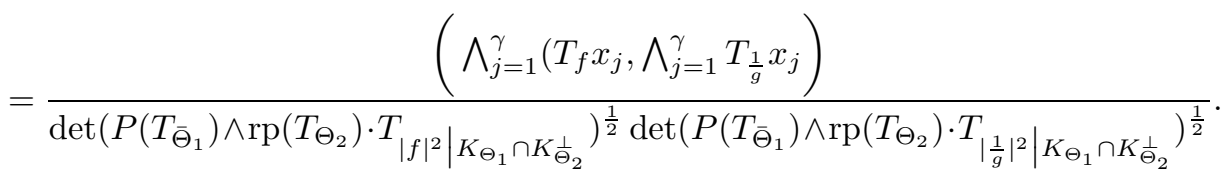

On the other hand,

$$
\left(\bigwedge_{j=1}^{\gamma}\left(T_{f} x_{j}, \bigwedge_{j=1}^{\gamma} T_{\frac{1}{g}} x_{j}\right)=\operatorname{det}\left(T_{\frac{f}{g}} x_{j}, x_{i}\right)=\operatorname{det}\left(\left.P\left(T_{\bar{\Theta}_{1}}\right) \wedge \operatorname{rp}\left(T_{\Theta_{2}}\right) \cdot T_{\frac{f}{g}}\right|_{K_{\Theta_{1}} \cap K_{\Theta_{2}}}\right) .\right.
$$

Combining the last two equations gives the desired result.

In order to compute $\sigma_{1, T_{\phi} T_{\phi}-1}$ in general we must remove the restriction that $m_{\alpha}=0$, for all $\alpha$.

Lemma 4.2. For small enough $t \neq 0 \in C$, the operator $P\left(T_{\bar{\Theta}_{1} \Theta_{2}}\right) T_{\frac{1}{g-t}} \mid \operatorname{ker} T_{\bar{\Theta}_{1} \Theta_{2}}$ is invertible.

Proof. $\sigma\left(\left.T_{\overline{\bar{g}-t}}\right|_{K_{\Theta_{1}}}\right)=\left\{\frac{1}{\bar{g}(z)-t}: z \in \Theta_{1}^{-1}(0)\right\}$; moreover for small $t \notin \sigma\left(\left.T_{\overline{\bar{g}}-t}\right|_{K_{\Theta_{1}}}\right)$, we have $P\left(T_{\bar{\Theta}_{1} \Theta_{2}}\right) T_{\frac{1}{\bar{g}-t} \mid \operatorname{ker} T_{\bar{\Theta}_{1} \Theta_{2}}}=\left.P\left(T_{\bar{\Theta}_{1} \Theta_{2}}\right)\left[g\left(\left.P\left(T_{\bar{\Theta}_{1}}\right) S\right|_{K_{\Theta_{1}}}\right)^{*}-t\right]^{-1}\right|_{\operatorname{ker} T_{\bar{\Theta}_{1} \Theta_{2}}}$. Consequently, $\operatorname{det}\left(P\left(T_{\bar{\Theta}_{1} \Theta_{2}}\right) T_{\bar{g}-t} \mid \operatorname{ker} T_{\bar{\Theta}_{2} \Theta_{1}}\right)$ is a non-zero function of $t$ analytic in a neighborhood of the origin. Thus it has at most an isolated zero at the origin.

Proposition 4.3. Suppose that $\left\{x_{j}\right\}_{1}^{\gamma}$ is any orthonormal basis for $\operatorname{ker} T_{\bar{\Theta}_{1} \Theta_{2}}$. Suppose that $\left\{v_{j}\right\}_{1}^{\gamma}$ is obtained from $\left\{T_{f} x_{j}\right\}_{1}^{\gamma}$ by the Gram-Schmidt procedure. Suppose also that $\left\{y_{j}\right\}_{1}^{\gamma}$ is obtained from $\left\{T_{\frac{1}{g}} x_{j}\right\}_{1}^{\gamma}$ by the Gram-Schmidt orthogonalization procedure. Then if $\left\{b_{j}\right\}_{1}^{\gamma}$ are the maximal root vectors associated to the orthonormal 
basis $\left\{v_{j}\right\}_{1}^{\gamma}$,

$$
\begin{gathered}
(-1)^{\sum_{\alpha=1}^{\gamma} m_{\alpha}} \frac{\prod_{\lambda \in \sigma\left(T_{\phi} T_{\phi}-1\right) \backslash\{0\}} \lambda}{\operatorname{det}\left(b_{j}, y_{k}\right)} \\
=\chi^{2}\left(P\left(T_{\bar{\Theta}_{1}}\right), P\left(T_{\bar{\Theta}_{2}}\right)\right) \cdot \prod_{|x|<1} c_{x}\left(\frac{f}{\bar{g}}, \frac{\Theta_{2}}{\Theta_{1}}\right) \cdot \operatorname{det}_{*} \partial\left\{T_{\bar{g}}+\mathcal{L}^{1}(H), T_{f}+\mathcal{L}^{1}(H)\right\} \\
\times \operatorname{det}\left(P\left(T_{\bar{\Theta}_{1}}\right) \wedge \operatorname{rp}\left(T_{\Theta_{2}}\right) \cdot T_{|f|^{2} \mid K_{\Theta_{1}} \cap K_{\Theta_{2}}^{\perp}}\right)^{\frac{1}{2}} \\
\cdot \operatorname{det}\left(P\left(T_{\bar{\Theta}_{1}}\right) \wedge \operatorname{rp}\left(T_{\Theta_{2}}\right) \cdot T_{\left|\frac{1}{g}\right|^{2} \mid K_{\Theta_{1}} \cap K_{\Theta_{2}}^{\perp}}\right)^{\frac{1}{2}} .
\end{gathered}
$$

Proof. Set $q(t)=\left.\operatorname{det} P\left(T_{\bar{\Theta}_{1} \Theta_{2}}\right) T_{\frac{1}{\bar{g}-t}}\right|_{\text {ker } T_{\bar{\Theta}_{1} \Theta_{2}}}$. By Lemma 4.2 for small $t$ the operator $\left.P\left(T_{\bar{\Theta}_{1} \Theta_{2}}\right) T_{\frac{1}{\bar{g}-t}}\right|_{\operatorname{ker} T_{\Theta_{1} \Theta_{2}}}$ is invertible. For small $t$ and $a \in C$ consider the two parameter family of operators

$$
\begin{gathered}
P\left(T_{\bar{\Theta}_{1} \Theta_{2}}\right) T_{\frac{f-a}{g-t}\left|\operatorname{ker} T_{\bar{\Theta}_{1} \Theta_{2}}=P\left(T_{\bar{\Theta}_{1} \Theta_{2}}\right) T_{\frac{f}{g-t}}\right| \operatorname{ker} T_{\bar{\Theta}_{1} \Theta_{2}}-a P\left(T_{\bar{\Theta}_{1} \Theta_{2}}\right) T_{\bar{g}-t} \mid \operatorname{ker} T_{\bar{\Theta}_{1} \Theta_{2}}} \\
=P\left(T_{\bar{\Theta}_{1} \Theta_{2}}\right) \frac{T_{\frac{1}{\bar{g}-t}} \mid \operatorname{ker} T_{\bar{\Theta}_{1} \Theta_{2}}}{q(t)}\left[q ( t ) \left(P\left(T_{\bar{\Theta}_{1} \Theta_{2}}\right) T_{\left.\frac{1}{\bar{g}-t} \mid \operatorname{ker} T_{\bar{\Theta}_{1} \Theta_{2}}\right)^{-1}}\right.\right. \\
\times P\left(T_{\bar{\Theta}_{1} \Theta_{2}}\right) T_{\left.\frac{f}{\bar{g}-t} \mid \operatorname{ker} T_{\bar{\Theta}_{1} \Theta_{2}}-q(t) a\right]}
\end{gathered}
$$

provided $q(t) \neq 0$.

Now $\left[q(t)\left(P\left(T_{\bar{\Theta}_{1} \Theta_{2}}\right) T_{\frac{1}{\bar{g}-t}} \mid \operatorname{ker} T_{\bar{\Theta}_{1} \Theta_{2}}\right)^{-1}\left(P\left(T_{\bar{\Theta}_{1} \Theta_{2}}\right) T_{\frac{f}{g}-t} \mid \operatorname{ker} T_{\bar{\Theta}_{1} \Theta_{2}}\right)-q(t) a\right]$ is analytic in $t$ in a neighborhood $\mathcal{N}$ of zero. Therefore,

$$
\operatorname{det} P\left(T_{\bar{\Theta}_{1} \Theta_{2}}\right) T_{\frac{f-a}{\bar{g}-t} \mid \operatorname{ker} T_{\bar{\Theta}_{1} \Theta_{2}}}=\frac{p(a, t)}{q(t)^{\gamma-1}}
$$

where $p(a, t)$ is a polynomial in $a$ of degree $\gamma$ with coefficients analytic in $t \in \mathcal{N}$. Since the divisor of the right-hand side is supported on a one-dimensional analytic variety, there exists a sequence of points $\left\{a_{n}, t_{n}\right\} \subset C^{2}$ converging to $(0,0)$ where $P\left(T_{\bar{\Theta}_{1} \Theta_{2}}\right) T_{\frac{f-a_{n}}{\bar{g}-t_{n}}} \mid \operatorname{ker} T_{\bar{\Theta}_{1} \Theta_{2}}$ is invertible.

For $t_{n} \in \mathcal{N}$ and small $\left|a_{n}\right|$, let $\phi_{n} \equiv \Theta_{1} \bar{\Theta}_{2}\left(f-a_{n}\right)\left(\bar{g}-t_{n}\right)$. The corresponding $m_{\alpha}=0$. Therefore, Proposition $4.2 \mathrm{v}$ ) holds for $\phi_{n}$. Since the right-hand side of Proposition $4.2 \mathrm{iv})$ is continuous in the spectral parameters $(t, a)$, it suffices to check the continuity of the left-hand side. To that end we shall use Proposition 2.1. So suppose $\left\{v_{j}^{(n)}\right\}_{1}^{\gamma}$ is obtained from $\left\{T_{f-a_{n}} x_{j}\right\}_{1}^{\gamma}$ by the Gram-Schmidt procedure and suppose also that $\left\{y_{j}^{(n)}\right\}_{1}^{\gamma}$ is similarly obtained from $\left\{T_{\frac{1}{g-t_{n}}} x_{j}\right\}_{1}^{\gamma}$. By Propositions 
4.3 and 4.2 part iv) we have for $n=1,2, \ldots$,

$$
\begin{gathered}
\operatorname{det}\left(T_{\phi_{n}} T_{\phi_{n}^{-1}}+\sum_{1}^{\gamma} y_{j}^{(n)} \otimes v_{j}^{(n)}\right) \\
=\chi^{2}\left(P\left(T_{\bar{\Theta}_{1}}\right), P\left(T_{\bar{\Theta}_{2}}\right)\right) \cdot \prod_{|x|<1} c_{x}\left(\frac{f-a_{n}}{\bar{g}-t_{n}}, \frac{\Theta_{1}}{\Theta_{2}}\right) \\
\cdot \operatorname{det}_{*} \partial\left\{T_{\bar{g}-t_{n}}+\mathcal{L}^{1}(H), T_{f-a_{n}}+\mathcal{L}^{1}(H)\right\} \\
\times \operatorname{det}\left(P\left(T_{\bar{\Theta}_{1}}\right) \wedge \operatorname{rp}\left(T_{\Theta_{2}}\right) \cdot T_{\left|f-a_{n}\right|^{2} \mid K_{\Theta_{1}} \cap K_{\Theta_{2}}}\right)^{\frac{1}{2}} \\
\cdot \operatorname{det}\left(P\left(T_{\bar{\Theta}_{1}}\right) \wedge \operatorname{rp}\left(T_{\Theta_{2}}\right) \cdot T_{\left|\frac{1}{g-t_{n}}\right|^{2} \mid K_{\Theta_{1}} \cap K_{\Theta_{2}}^{\perp}}\right)^{\frac{1}{2}} .
\end{gathered}
$$

Now suppose $\left\{h_{j}^{(n)}\right\}_{1}^{\gamma}$ is a sequence of linearly independent sets of vectors so that $h_{j}^{(n)} \rightarrow h_{j}$ for each $j=1,2, \ldots, \gamma$. Suppose also that $\left\{h_{j}\right\}_{1}^{\gamma}$ is linearly independent with associated orthonormal vectors $\left\{z_{j}\right\}_{1}^{\gamma}$. For each $n=1,2, \ldots$, we can apply the Gram-Schmidt procedure to $\left\{h_{j}^{(n)}\right\}_{1}^{\gamma}$ to get vectors $\left\{z_{j}^{(n)}\right\}_{1}^{\gamma}$.

We claim that $z_{j}^{(n)} \rightarrow z_{j}$, for $j=1,2, \ldots, \gamma$. To check this, note that

$$
\begin{gathered}
z_{1}^{(n)}=\frac{h_{1}^{(n)}}{\left\|h_{1}^{(n)}\right\|} \rightarrow \frac{h_{1}}{\left\|h_{1}\right\|}=z_{1}, \\
z_{2}^{(n)}=\frac{h_{2}^{(n)}-\left(h_{2}^{(n)}, z_{1}^{(n)}\right) z_{1}^{(n)}}{\left\|h_{2}^{(n)}\right\|} \rightarrow \frac{h_{2}-\left(h_{2}, z_{1}\right) z_{1}}{\left\|h_{2}\right\|}=z_{2} .
\end{gathered}
$$

So in general,

$$
z_{j}^{(n)}=\frac{h_{j}^{(n)}-\sum_{1 \leq i<j}\left(h_{j}^{(n)}, z_{i}^{(n)}\right) z_{i}^{(n)}}{\left\|h_{j}^{(n)}-\sum_{1 \leq i<j}\left(h_{j}^{(n)}, z_{i}^{(n)}\right) z_{i}^{(n)}\right\|} \rightarrow \frac{h_{j}-\sum_{1 \leq i<j}\left(h_{j}, z_{i}\right) z_{i}}{\left\|h_{j}-\sum_{1 \leq i<j}\left(h_{j}, z_{i}\right) z_{i}\right\|}=z_{j} .
$$

Now with $h_{j}^{(n)}=T_{f-a_{n}} x_{j}$, we get $v_{j}^{(n)}=z_{j}^{(n)} \rightarrow z_{j}=v_{j}$. Similarly, if $h_{j}^{(n)}=$ $T_{\frac{1}{g-t_{n}}} x_{j}$, then $y_{j}^{(n)}=z_{j}^{(n)} \rightarrow z_{j}=y_{j}$. Therefore,

$$
\sum_{j=1}^{\gamma} y_{j}^{(n)} \otimes v_{j}^{(n)} \rightarrow \sum_{j=1}^{\gamma} y_{j} \otimes v_{j} \quad \text { in trace norm. }
$$

Lemma 4.3. If $\left\{b_{j}\right\}_{1}^{\gamma}$ are maximal vectors associated to the orthonormal basis $\left\{v_{j}\right\}_{1}^{\gamma}$, then

$$
\lim _{n \rightarrow \infty} \frac{\prod_{\lambda \in \sigma\left(T_{\phi_{n}} T_{\phi_{n}}\right) \backslash\{0\}} \lambda}{\operatorname{det}\left(v_{j}^{(n)}, y_{k}^{(n)}\right)}=(-1)^{\sum_{\alpha=1}^{\gamma} m_{\alpha}} \frac{\prod_{\lambda \in \sigma\left(T_{\phi} T_{\phi}-1\right) \backslash\{0\}} \lambda}{\operatorname{det}\left(b_{j}, y_{k}\right)} .
$$

Proof. By Proposition 2.1 it suffices to show that

$$
\lim _{n \rightarrow \infty} \operatorname{det}\left(T_{\phi_{n}} T_{\phi_{n}^{-1}}+\sum_{j=1}^{\gamma} y_{j}^{(n)} \otimes v_{j}^{(n)}\right)=\operatorname{det}\left(T_{\phi} T_{\phi^{-1}}+\sum_{j=1}^{\gamma} y_{j} \otimes v_{j}\right) .
$$

Since the dyadic sums converge in $\mathcal{L}^{1}(H)$ it remains to check that $1-T_{\phi_{n}} T_{\phi_{n}}^{-1}$ converges to $1-T_{\phi} T_{\phi^{-1}}$ in trace norm.

Note that the Hankel operators $H_{\Theta_{1} \bar{\Theta}_{2}}$ and $H_{\bar{\Theta}_{1} \tilde{\Theta}_{2}}$ are trace class. 
Let $\psi=\Theta_{1} \bar{\Theta}_{2} \cdot \omega \bar{\eta}$, where $\omega, \eta$ are outer in $K_{2,2}^{\frac{1}{2}, \frac{1}{2}} \cap \mathcal{W}$. Then

$$
T_{\psi} T_{\psi^{-1}}=\left[T_{\Theta_{1} \bar{\Theta}_{2}} T_{\omega \bar{\eta}}+H_{\Theta_{1} \bar{\Theta}_{2}} H_{\tilde{\omega} \bar{\eta}}\right]\left[T_{\omega^{-1} \bar{\eta}^{-1}} T_{\bar{\Theta}_{1} \Theta_{2}}+H_{\omega^{-1} \bar{\eta}^{-1}} H_{\tilde{\Theta}_{1} \tilde{\Theta}_{2}}\right] .
$$

With $\omega=f-a_{n}$ and $\eta=g-\bar{t}_{n}$ we have $H_{\tilde{\omega} \tilde{\eta}} \rightarrow H_{\tilde{f} \tilde{\tilde{g}}}$ and $H_{\omega^{-1} \bar{\eta}^{-1}} \rightarrow H_{f^{-1} \bar{g}^{-1}}$ in the uniform norm. Also, $T_{\Theta_{1} \bar{\Theta}_{2}}\left[T_{\omega \bar{\eta}} T_{\omega^{-1} \bar{\eta}^{-1}}\right] T_{\bar{\Theta}_{1} \Theta_{2}}=T_{\Theta_{1} \bar{\Theta}_{2}}\left[1+\left[T_{\bar{\eta}}, T_{\omega}\right] T_{\bar{\eta}^{-1}} T_{\omega^{-1}}\right] T_{\bar{\Theta}_{1} \Theta_{2}}$. But $\left[T_{\bar{\eta}}, T_{\omega}\right]=\left[T_{\bar{g}}, T_{f}\right] \in \mathcal{L}^{1}\left(H^{2}(\mathbb{T})\right)$. Since $T_{\bar{\eta}^{-1}} T_{\omega^{-1}} \rightarrow T_{\bar{g}}^{-1} T_{f^{-1}}$ in the uniform norm it follows that $1-T_{\phi_{n}} T_{\phi_{n}}^{-1}$ converges to $1-T_{\phi} T_{\phi^{-1}}$ in trace norm.

To complete the proof of Theorem 1.6 we note that when the zeros of $\Theta_{1}$ and $\Theta_{2}$ are simple, the equality

$$
\begin{aligned}
& \chi^{2}\left(P\left(T_{\bar{\Theta}_{1}}\right), P\left(T_{\bar{\Theta}_{2}}\right)\right)=\frac{\prod_{1 \leq \alpha, \beta \leq \ell}\left|1-\bar{\mu}_{\alpha} \mu_{\beta}\right|}{\prod_{\substack{1 \leq \alpha \leq \ell \\
1 \leq \beta \leq q}}\left|1-\bar{\mu}_{\alpha} \nu_{\beta}\right|^{2}} \mid \sum_{N} \frac{\prod_{\substack{\mu_{\alpha} \in \bar{N} \\
1 \leq \beta \leq q}}\left(\mu_{\alpha}-\nu_{\beta}\right)}{\prod_{\substack{\mu_{\alpha} \in N \\
\mu_{\beta} \in \bar{N}}}\left(\mu_{\alpha}-\mu_{\beta}\right)}
\end{aligned}
$$

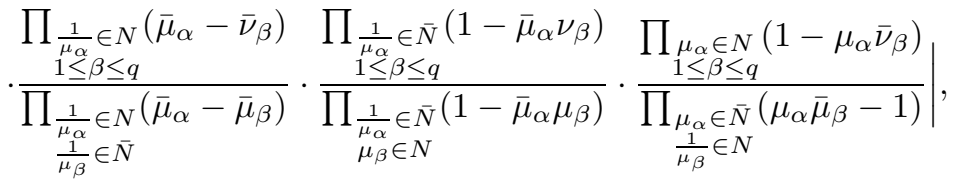

with the sum extended over all subsets $N \subset\left\{\mu_{1}, \cdots, \mu_{\ell} ; \frac{1}{\bar{\mu}_{1}}, \cdots, \frac{1}{\bar{\mu}_{\ell}}\right\}$ of cardinality $\ell$, and $\bar{N}=\left\{\mu_{1}, \cdots, \mu_{\ell} ; \frac{1}{\bar{\mu}_{1}}, \cdots, \frac{1}{\bar{\mu}_{\ell}}\right\} \backslash N$ is proved in Proposition 3.4 of [17.

Corollary 4.1. Let $\left\{x_{j}\right\}_{1}^{\gamma}$ be an orthonormal basis for $\operatorname{ker} T_{\bar{\Theta}_{1} \Theta_{2}}$. Let $\left\{u_{j}\right\}_{1}^{\gamma}$ be the top root vectors of the associated Jordan chain lying over $T_{f} x_{j}$. Then

$$
\begin{aligned}
\sigma_{1, T_{\phi} T_{\phi}-1}= & (-1)^{\sum_{\alpha=1}^{\gamma} m_{\alpha}} \prod_{\lambda \in \sigma\left(T_{\phi} T_{\phi}-1\right) \backslash\{0\}} \lambda \cdot \frac{\left\|\bigwedge T_{\frac{1}{g} \mid \operatorname{ker} T_{\bar{\Theta}_{1} \Theta_{2}}}\right\|^{2}}{\operatorname{det}\left(u_{j}, T_{\frac{1}{g}} x_{\tau}\right)} \\
& \left.\cdot\left[\bigwedge T_{f} x_{j} \otimes \bigwedge T_{\frac{1}{g}} x_{j}+\operatorname{ran} T_{\phi}\right]\right)^{*},
\end{aligned}
$$

ii)

$$
\begin{aligned}
& (-1)^{\sum_{\alpha=1}^{\gamma} m_{\alpha}} \frac{\prod_{\lambda \in \sigma\left(T_{\phi} T_{\phi}-1\right) \backslash\{0\}} \lambda}{\operatorname{det}\left(u_{j}, T_{\frac{1}{g}} x_{\tau}\right)}=\chi^{2}\left(P\left(T_{\bar{\Theta}_{1}}\right), P\left(T_{\bar{\Theta}_{2}}\right)\right) \prod_{\{x:\|x\|<1\}} c_{x}\left(\frac{f}{\bar{g}}, \frac{\Theta_{2}}{\Theta_{1}}\right) \\
& \cdot \operatorname{det}_{*} \partial\left\{T_{\bar{g}}+\mathcal{L}^{1}, T_{f}+\mathcal{L}^{1}\right\} .
\end{aligned}
$$

\section{Proofs of Theorem 1.1 Part ii) and Theorem 1.3}

It is of interest to estimate the speed of convergence in our limit theorems. The proofs of Theorem 1.1 part ii) and Theorem 1.3 are based on a series of lemmas with numerical estimates. In what follows $\|\cdot\|_{2}$ denotes the Hilbert-Schmidt norm and $\|\cdot\|_{1}$ denotes the trace norm.

Lemma 5.1. Suppose $f \in \operatorname{Lip}_{\beta}, \beta>\frac{1}{2}$. Then

$$
\left\|S^{* n+1} \operatorname{PfQ}\right\|_{2} \leq\left(\frac{4 \pi}{3}\right)^{\beta}\left(\frac{1}{1-\left(\frac{1}{2}\right)^{2 \beta}}\right)^{\frac{1}{2}}\left(\frac{1}{2 \beta-1}\right)^{\frac{1}{2}}\|f\|_{\operatorname{Lip}_{\beta}} \cdot(n+1)^{\frac{1-2 \beta}{2}} .
$$


Proof. The proof ultimately depends upon the Bernstein inequality; cf. p. 32, Katznelson [27. It is known 5 that

$$
\sum_{2^{m} \leq k \leq 2^{m+1}}|\hat{f}(k)|^{2} \leq\left(\frac{2 \pi}{3 \cdot 2^{m}}\right)^{2 \beta}\|f\|_{\operatorname{Lip}_{\beta}}^{2} .
$$

So, if $2^{m} \leq n \leq 2^{m+1}$, we have

$$
\begin{gathered}
\sum_{n \leq k}|\hat{f}(k)|^{2} \leq\left(\frac{2 \pi}{3}\right)^{2 \beta}\|f\|_{\operatorname{Lip}_{\beta}}^{2} \sum_{r \geq m}\left(\frac{1}{2^{r}}\right)^{2 \beta} \\
=\left(\frac{2 \pi}{3}\right)^{2 \beta}\|f\|_{\operatorname{Lip}_{\beta}}^{2}\left(\frac{1}{2^{m}}\right)^{2 \beta} \frac{1}{1-\left(\frac{1}{2}\right)^{2 \beta}} .
\end{gathered}
$$

Hence $\sum_{n \leq k}|\hat{f}(k)|^{2} \leq \frac{\Omega}{n^{2 \beta}}$, where $\Omega=\left(\frac{4 \pi}{3}\right)^{2 \beta}\left(\frac{1}{1-\left(\frac{1}{2}\right)^{2 \beta}}\right)\|f\|_{\operatorname{Lip}_{\beta}}^{2}$.

Now,

$$
\begin{gathered}
\left\|S^{* n+1} P M_{f} Q\right\|_{2}^{2}=\sum_{k \geq 1} k|\hat{f}(k+n+1)|^{2} \\
=\sum_{k \geq 1}|\hat{f}(k+n+1)|^{2}+\sum_{k \geq 1}|\hat{f}(k+n+2)|^{2}+\sum_{k \geq 1}|\hat{f}(k+n+3)|^{2}+\cdots \\
\leq \Omega\left[\frac{1}{(n+2)^{2 \beta}}+\frac{1}{(n+3)^{2 \beta}}+\frac{1}{(n+4)^{2 \beta}}+\cdots\right] \leq \frac{\Omega}{(2 \beta-1)}(n+1)^{1-2 \beta} .
\end{gathered}
$$

Since $S^{* \gamma} T_{\frac{1}{f}} T_{\frac{1}{\bar{g}}}\left[1-P\left(T_{\bar{\phi}}\right)\right]=R\left(T_{\phi}\right)$, it follows that for $n \gg 0$,

$$
\operatorname{ker}\left[S^{*} n+1 R\left(T_{\phi}\right) S^{n+1}\right]=\operatorname{ran}\left\{S^{* n+1} T_{\frac{1}{f}} T_{\frac{1}{g}}\left[1-P\left(T_{\bar{\phi}}\right)\right] S^{n+1}\right\}^{-1} P_{\gamma} .
$$

Moreover, $\left\{S^{* n+1} T_{\frac{1}{f}} T_{\frac{1}{\bar{g}}}\left[1-P\left(T_{\bar{\phi}}\right)\right] S^{n+1}\right\}^{-1}$ converges uniformly to $T_{f} T_{\bar{g}}$.

Lemma 5.2. Suppose $\phi=z^{\gamma} \psi$ with $\psi \in \operatorname{Lip}_{\beta}, \frac{1}{2}<\beta<1$, and $T_{\psi}$ is invertible. Set $J_{n}=S^{* n+1} T_{\psi}^{-1}\left[1-P\left(T_{\bar{\phi}}\right)\right] S^{n+1}$. Then

$$
\begin{gathered}
\left\|S^{* n+1} R\left(T_{\phi}\right) S^{n+1}-T_{\phi^{-1}}\right\|=O\left(n^{-2 \beta}\right) . \\
\left\|J_{n}-T_{\psi^{-1}}\right\|=O\left(n^{-2 \beta}\right) \quad \text { and } \quad\left\|J_{n}-T_{\psi^{-1}}\right\|_{1}=O\left(n^{1-2 \beta}\right) .
\end{gathered}
$$

In particular, for $n \gg 0, J_{n}$ is invertible and

$$
\begin{aligned}
& \operatorname{ker} S^{* n+1} R\left(T_{\phi}\right) S^{n+1}=J_{n}^{-1} P_{\gamma-1}\left(H^{2}(\mathbb{T})\right) . \\
& \left\|J_{n}^{-1} T_{\frac{1}{\bar{g}}} e_{\alpha}-T_{f} e_{\alpha}\right\|=O\left(n^{-2 \beta}\right) \quad \text { for } \quad \alpha=0,1,2, \ldots, \gamma-1 .
\end{aligned}
$$

Proof. We have $R\left(T_{\phi}\right)=S^{* \gamma} T_{\psi}^{-1}\left[1-P\left(T_{\bar{\phi}}\right)\right]$, and $\operatorname{ker} P\left(T_{\bar{\phi}}\right)=\left\{\bigvee_{\alpha=0}^{\gamma-1} \frac{1}{g} e_{\alpha}\right\}$ where $\psi=f \bar{g}, f, g \in H^{\infty} \cap \operatorname{Lip}_{\beta}$. In particular, $\left\|P\left(T_{\bar{\phi}}\right) S^{n+1}\right\|=O\left(n^{-\beta}\right)$.

The fact that $f, g \in \operatorname{Lip}_{\beta} \cap H^{\infty}$ follows because $T_{\psi}$ is invertible and the conjugate function is bounded on $\operatorname{Lip}_{\beta}, 0<\beta<1$; cf. [28]. Accordingly, there are polynomials

\footnotetext{
${ }^{5}$ See for example equation (6.4), Katznelson.
} 
$p_{n}$ and $q_{n}$ of degree $\leq n$ so that $\left\|\frac{1}{f}-p_{n}\right\|_{\infty}$ and $\left\|\frac{1}{g}-q_{n}\right\|_{\infty}$ are $O\left(n^{-\beta}\right)$. Also, since $S^{* n+1} T_{\psi}^{-1} S^{n+1}=T_{\psi^{-1}}-S^{* n+1} P \frac{1}{f} Q \frac{1}{\bar{g}} S^{n+1}$, we have

$$
\begin{gathered}
J_{n}=S^{* n+1} T_{\psi}^{-1}\left[1-P\left(T_{\bar{\phi}}\right)\right] S^{n+1}=S^{* n+1} T_{\psi}^{-1} S^{n+1}-S^{* n+1} T_{\psi}^{-1} P\left(T_{\bar{\phi}}\right) S^{n+1} \\
=T_{\psi^{-1}}-S^{* n+1} P \frac{1}{f} Q \frac{1}{\bar{g}} P S^{n+1}-S^{* n+1} T_{\psi^{-1}} P\left(T_{\bar{\phi}}\right) S^{n+1}+S^{* n+1} P \frac{1}{f} Q \frac{1}{\bar{g}} P\left(T_{\bar{\phi}}\right) S^{n+1} .
\end{gathered}
$$

Also

$$
\begin{gathered}
\left\|S^{* n+1} P \frac{1}{f} Q \frac{1}{\bar{g}} P S^{n+1}\right\| \leq\left\|S^{* n+1} P \frac{1}{f} Q\right\|\left\|Q \frac{1}{\bar{g}} P S^{n+1}\right\| \\
=\left\|S^{* n+1} P\left(\frac{1}{f}-p_{n}\right) Q\right\|\left\|Q\left(\frac{1}{\bar{g}}-\bar{q}_{n}\right) P S^{n+1}\right\| \leq\left\|\frac{1}{f}-p_{n}\right\|_{\infty}\left\|\frac{1}{g}-q_{n}\right\|_{\infty}=O\left(n^{-2 \beta}\right),
\end{gathered}
$$

and $\left\|S^{* n+1} P \frac{1}{f} Q \frac{1}{\bar{g}} S^{n+1}\right\|_{1} \leq\left\|S^{* n+1} P \frac{1}{f} Q\right\|_{2}\left\|S^{* n+1} P \frac{1}{\bar{g}} Q\right\|_{2}=O\left(n^{1-2 \beta}\right)$.

Now, $S^{* n+1} T_{\psi^{-1}}=T_{\frac{1}{\bar{g}}} S^{* n+1} T_{\frac{1}{f}}$, and $\operatorname{ker} T_{\bar{\phi}}=\bigvee_{\alpha=0}^{\gamma-1}\left\{\frac{1}{g} e_{\alpha}\right\}$, ran $T_{\frac{1}{f}} P\left(T_{\bar{\phi}}\right)=$ $\bigvee_{\alpha=0}^{\gamma-1}\left\{\frac{1}{f g} e_{\alpha}\right\}$. Since $\frac{1}{f}, \frac{1}{g} \in \operatorname{Lip}_{\beta}$ it follows that

$$
\left\|S^{* n+1} T_{\psi^{-1}} P\left(T_{\bar{\phi}}\right) S^{n+1}\right\|_{1}=O\left(n^{-2 \beta}\right) .
$$

Therefore,

$$
\left\|J_{n}-T_{\psi^{-1}}\right\|=O\left(n^{-2 \beta}\right) \quad \text { and } \quad\left\|J_{n}-T_{\psi^{-1}}\right\|_{1}=O\left(n^{1-2 \beta}\right) .
$$

The fact that $\operatorname{ker} S^{* n+1} R\left(T_{\phi}\right) S^{n+1}=J_{n}^{-1} P_{k-1}\left(H^{2}(\mathbb{T})\right)$ follows immediately since $S^{* n+1} R\left(T_{\phi}\right) S^{n+1}=S^{* k} J_{n}$. Because $\left\|J_{n}-T_{\psi^{-1}}\right\|=O\left(n^{-2 \beta}\right)$ and $T_{\psi^{-1}}$ is invertible we have $\left\|J_{n}^{-1}-T_{\psi^{-1}}^{-1}\right\|=O\left(n^{-2 \beta}\right)$. Furthermore, since for all $\alpha$ we have $T_{\psi^{-1}}^{-1} T_{\frac{1}{9}} e_{\alpha}=$ $T_{f} e_{\alpha}$, we have $\left\|J_{n}^{-1} T_{\frac{1}{\bar{g}}} e_{\alpha}-T_{f} e_{\alpha}\right\|=O\left(n^{-2 \beta}\right)$.

\section{Lemma 5.3.}

$$
\left\|R\left(T_{\phi} S^{* n+1} R\left(T_{\phi}\right) S^{n+1}\right)-R\left(T_{\phi} T_{\phi^{-1}}\right)\right\|=O\left(n^{-2 \beta}\right) .
$$

Proof. Set $Y_{n}=T_{\phi} S^{* n+1} R\left(T_{\phi}\right) S^{n}$ and $Y=T_{\phi} T_{\phi^{-1}}$. For $n \gg 0, S^{* n+1} R\left(T_{\phi}\right) S^{n+1}$ is surjective. Hence $\operatorname{rp}\left(Y_{n}\right)=1-P\left(T_{\bar{\phi}}\right)$. Therefore, since $R\left(Y_{n}^{*}\right)=\left[Y_{n} Y_{n}^{*}+\right.$ $\left.P\left(T_{\bar{\phi}}\right)\right]^{-1} Y_{n}$, we have $\left\|R\left(Y_{n}^{*}\right)-R\left(Y^{*}\right)\right\|=O\left(n^{-2 \beta}\right)$. Consequently, since $R(A)^{*}=$ $R\left(A^{*}\right)$, we have

$$
\left\|R\left(Y_{n}\right)-R(Y)\right\|=\left\|R\left(Y_{n}\right)^{*}-R(Y)^{*}\right\|=\left\|R\left(Y_{n}^{*}\right)-R\left(Y^{*}\right)\right\|=O\left(n^{-2 \beta}\right) .
$$

Fix an orthonormal basis $\left\{y_{\tau}\right\}_{\tau=1}^{\gamma}$ of $\operatorname{ker}\left(T_{\bar{\phi}}\right)$. Suppose $n \gg 0$ so that $\operatorname{dim} \operatorname{ker}\left(S^{* n+1} R\left(T_{\phi}\right) S^{n+1}\right)=\gamma$ and $J_{n}$ is invertible. For $\alpha=1,2, \ldots, \gamma$ set $w_{\alpha}^{(n)}=$ $J_{n}^{-1} T_{\frac{1}{9}} e_{\alpha}$ and let $\left\{v_{\alpha}^{(n)}\right\}_{1}^{\gamma}$ be obtained from $\left\{w_{\alpha}^{(n)}\right\}_{1}^{\gamma}$ by the Gram-Schmidt orthogonalization procedure. Note that $\left\|w_{\alpha}^{(n)}-f e_{\alpha}\right\|=O\left(n^{-2 \beta}\right)$.

As above let $\mathcal{Z}$ denote the subspace of $H^{2}(\mathbb{T})$ given by $\operatorname{ker}\left(T_{\bar{\phi}}\right) \oplus \bigvee\left\{\left(T_{\phi} T_{\phi^{-1}}\right)^{r} u_{\alpha}\right.$ : $\left.m_{\alpha} \geq r \geq 1, \alpha=1,2, \ldots, \gamma\right\}$, where $\left\{u_{\alpha}\right\}$ is any set of maximal root vectors for $\left\{T_{f} e_{\alpha}\right\}_{1}^{\gamma}$. Let $K_{n}$ denote the operator on $\mathcal{Z}$ where

$$
\begin{gathered}
K_{n}\left(y_{\tau}\right)=P_{\mathcal{Z}}\left(v_{\alpha}^{(n)}\right) \\
K_{n}\left(T_{\phi} T_{\phi^{-1}}\right)^{r} u_{\alpha}=P_{\mathcal{Z}} R\left(T_{\phi} S^{* n+1} R\left(T_{\phi}\right) S^{n+1}\right)\left(T_{\phi} T_{\phi^{-1}}\right)^{r} u_{\alpha} .
\end{gathered}
$$


By (ii) of Lemma 5.2 and the continuity of the Gram-Schmidt procedure, $v_{\alpha}^{(n)} \rightarrow$ $v_{\alpha}$ where $\left\{v_{\alpha}^{(n)}\right\}_{1}^{\gamma}$ is obtained from $\left\{T_{f} e_{\alpha}\right\}_{1}^{\gamma}$ by applying the Gram-Schmidt orthogonalization. Let $K$ denote the limit operator on $\mathcal{Z}$ so that

$$
\begin{gathered}
K\left(y_{\tau}\right)=P_{\mathcal{Z}} v_{\tau}, \\
K\left(T_{\phi} T_{\phi^{-1}}\right)^{r} u_{\alpha}=P_{\mathcal{Z}}\left[1-P\left(T_{\phi} T_{\phi^{-1}}\right)\right]\left(T_{\phi} T_{\phi^{-1}}\right)^{r-1} u_{\alpha} .
\end{gathered}
$$

Lemma 5.4. $\left\|K_{n}-K\right\|=O\left(n^{-2 \beta}\right)$.

Proof. Since $\left\|J_{n}^{-1} T_{\frac{1}{\bar{g}}} e_{\alpha}-T_{f} e_{\alpha}\right\|=O\left(n^{-2 \beta}\right)$, computation shows that $\left\|v_{\alpha}^{(n)}-v_{\alpha}\right\|=$ $O\left(n^{-2 \beta}\right)$. Now, comparing $K_{n}$ and $K$ on basis elements of $Z$, we have with $z_{r_{\alpha}}(n)=$ $X_{n}^{-1}\left(T_{\phi} T_{\phi^{-1}}\right)^{r} u_{\alpha}$ as in $\S 3$, for $j=1,2, \ldots, \gamma$,

$$
\left\|\left(K_{n}-K\right) y_{j}\right\|=\left\|P_{\mathcal{Z}}\left(v_{\alpha}^{(n)}-v_{\alpha}\right)\right\|=O\left(n^{-2 \beta}\right) .
$$

Also, on $\left(T_{\phi} T_{\phi^{-1}}\right)^{r} u_{\alpha}$ we have

$$
\begin{aligned}
{\left[K_{n}-K\right]\left(T_{\phi} T_{\phi^{-1}}\right)^{r} u_{\alpha}=} & P_{\mathcal{Z}}\left[1-P\left(S^{* n+1} R\left(T_{\phi}\right) S^{n+1}\right)\right] z_{r_{\alpha}}(n) \\
& -P_{\mathcal{Z}}\left[1-P\left(T_{\phi^{-1}}\right)\right]\left(T_{\phi} T_{\phi^{-1}}\right)^{r-1} u_{\alpha} .
\end{aligned}
$$

Now,

$$
\left[1-P\left(S^{* n+1} R\left(T_{\phi}\right) S^{n+1}\right)\right] z_{r_{\alpha(n)}}=R\left[T_{\phi} S^{* n+1} R\left(T_{\phi}\right) S^{n+1}\right]\left(T_{\phi} T_{\phi^{-1}}\right)^{r} u_{\alpha} .
$$

Hence, by Lemma 5.3 we have

$$
\begin{gathered}
\left\|\left[K_{n}-K\right]\left(T_{\phi} T_{\phi}^{-1}\right)^{r} u_{\alpha}\right\|=\| P_{\mathcal{Z}}\left\{R\left(T_{\phi} S^{* n+1} R\left(T_{\phi}\right) S^{n+1}\right)\left(T_{\phi} T_{\phi^{-1}}\right)\right. \\
\left.-\left[1-P\left(T_{\phi^{-1}}\right)\right]\right\}\left(T_{\phi} T_{\phi^{-1}}\right)^{r-1} u_{\alpha} \| \\
=\left\|P_{\mathcal{Z}}\left\{R\left(T_{\phi} S^{* n+1} R\left(T_{\phi}\right) S^{n+1}\right)-R\left(T_{\phi} T_{\phi^{-1}}\right)\right\}\left(T_{\phi} T_{\phi^{-1}}\right)^{r} u_{\alpha}\right\|=O\left(n^{-2 \beta}\right) .
\end{gathered}
$$

Consequently,

$$
\left\|K_{n}-K\right\|=O\left(n^{-2 \beta}\right) .
$$

Recall that for $n \gg 0$,

$$
D_{n}\left(T_{\phi}\right)=\mathbf{G}(\phi)^{n+1} \frac{\operatorname{det}\left(P_{\mathcal{X}} X_{n \mid \mathcal{X}}\right)}{\operatorname{det} K_{n}} \cdot \operatorname{det}\left(S^{n+1} v_{\alpha}^{(n)}, y_{\tau}\right) .
$$

Now

$$
\begin{gathered}
\left\|X_{n}-T_{\phi} T_{\phi^{-1}}\right\|_{1}=\left\|T_{\phi} S^{* \gamma} J_{n}-P\left(T_{\bar{\phi}}\right) S^{n+1}-T_{\phi} S^{* \gamma} T_{\psi^{-1}}\right\|_{1} \\
\leq\left\|T_{\phi} S^{* \gamma}\left[J_{n}-T_{\psi^{-1}}\right]\right\|_{1}+\left\|P\left(T_{\bar{\phi}}\right) S^{n+1}\right\|_{1}=O\left(n^{1-2 \beta}\right) .
\end{gathered}
$$

Using the inequality

$$
|\operatorname{det}[1+M]-\operatorname{det}[1+N]| \leq\|M-N\|_{1} \exp \left(\|M\|_{1}+\|N\|_{1}+1\right),
$$

we see that

$$
\begin{gathered}
\operatorname{det} P_{\mathcal{X}} X_{n} P_{\mathcal{X}}=\operatorname{det}\left[P_{\mathcal{X}} T_{\phi} T_{\phi^{-1}} \mid \mathcal{X}\right]\left[1+O\left(n^{1-2 \beta}\right)\right] \\
=\prod_{\lambda \in \sigma\left(T_{\phi} T_{\phi^{-1}} \backslash\{0\}\right.} \lambda\left[1+O\left(n^{1-2 \beta}\right)\right] .
\end{gathered}
$$


Also by Lemmas 5.2 and 5.4

$$
\frac{\operatorname{det}\left(S^{n+1} v_{\alpha}^{(n)}, y_{\tau}\right)}{\operatorname{det} K_{n}}=(-1)^{\sum_{1}^{\gamma} m_{\alpha}} \cdot \frac{\operatorname{det}\left(S^{n+1} w_{\alpha}^{(n)}, y_{\tau}\right)}{\operatorname{det}\left(u_{\alpha}, y_{\tau}\right)} \cdot\left[1+O\left(n^{-2 \beta}\right)\right] .
$$

Consequently, switching vectors $\left\{y_{\tau}\right\}_{1}^{\gamma}$ to an arbitrary basis $\left\{t_{\tau}\right\}_{1}^{\gamma}$ of $\operatorname{ker} T_{\bar{\phi}}$, we have

$D_{n}\left(T_{\phi}\right)=\mathbf{G}(\phi)^{n+1} \operatorname{det}\left(S^{n+1} w_{\alpha}^{(n)}, t_{\tau}\right) \frac{(-1)^{\sum_{1}^{\gamma} m_{\alpha}} \prod_{\lambda \in \sigma\left(T_{\phi} T_{\phi^{-1}}\right) \backslash\{0\}} \lambda}{\operatorname{det}\left(u_{\alpha}, t_{\tau}\right)}\left[1+O\left(n^{1-2 \beta}\right)\right]$.

This proves statement ii) of Theorem 1.1. We now complete the proof of Theorem 1.3 .

Lemma 5.5.

$$
\begin{gathered}
\operatorname{det}\left(S^{n+1} w_{\alpha}^{(n)}, T_{\frac{1}{g}} e_{\tau}\right) \\
=\frac{1}{\bar{g}(0)^{\gamma}} \operatorname{det}\left(S^{n+1}\left(S^{* n+1-\gamma} T_{\psi}^{-1} S^{n+1-\gamma}\right)^{-1} e_{\alpha}, T_{\frac{1}{g}} e_{\tau}\right)\left[1+O\left(n^{-2 \beta}\right)\right] .
\end{gathered}
$$

Proof. It is enough to prove the statement with $T_{\frac{1}{g}} e_{\alpha}$ replaced by any basis of $\operatorname{ker} T_{\bar{\phi}}$.

Let $\left\{y_{\tau}\right\}_{1}^{\gamma}$ be an orthonormal basis for ker $T_{\bar{\phi}}$. Since $S^{n+1} w_{\alpha}^{(n)}=S^{n+1} J_{n}^{-1} T_{\overline{1}} e_{\alpha} \in$ $\operatorname{ker}\left[T_{\bar{\phi}} \oplus \operatorname{ran} T_{\phi} P_{n}\right] \cap \operatorname{ran} S^{n+1}$, there are complex numbers $\left\{\lambda_{n, \alpha, \tau}\right\}_{1 \leq \alpha, \tau \leq \gamma}$ and a vector $g_{n \alpha} \in \operatorname{ran}\left(T_{\phi} P_{n}\right)$ so that

$$
S^{n+1} w_{\alpha}^{(n)}=\sum_{\tau=1}^{\gamma} \lambda_{n, \alpha, \tau} y_{\tau}+T_{\phi} g_{n \alpha},
$$

where $\lambda_{n, \alpha, \tau}=\left(S^{n+1} w_{\alpha}^{(n)}, y_{\tau}\right)$. Since $T_{\phi}=T_{\psi} S^{\gamma}$ and $S^{* \gamma} T_{\psi} S^{\gamma}=T_{\psi}$, we have for $n \gg 0$

$$
S^{* n+1} T_{\psi}^{-1} S^{n+1-\gamma} w_{\alpha}^{(n)}=\sum_{\tau=1}^{\gamma} \lambda_{n, \alpha, \tau} S^{* n+1} T_{\psi}^{-1} S^{* \gamma} y_{\tau} .
$$

Multiplying by $S^{\gamma}$ and rewriting gives

$$
\begin{aligned}
S^{* n+1-\gamma} T_{\psi}^{-1} S^{n+1-\gamma} w_{\alpha}^{(n)}= & \sum_{\tau=1}^{\gamma}\left(S^{* n+1-\gamma} T_{\psi}^{-1} S^{n+1-\gamma} w_{\alpha}^{(n)}, e_{\tau}\right) e_{\tau} \\
& +\sum_{\tau=1}^{\gamma} \lambda_{n, \alpha, \tau} S^{\gamma} S^{* n+1} T_{\psi}^{-1} S^{* \gamma} y_{\tau} .
\end{aligned}
$$

Applying the inverse of $S^{* n+1-\gamma} T_{\psi}^{-1} S^{n+1-\gamma}$ to both sides gives

$$
\begin{aligned}
w_{\alpha}^{(n)} & =\sum_{\tau=1}^{\gamma}\left(S^{* n+1-\gamma} T_{\psi}^{-1} S^{n+1-\gamma} w_{\alpha}^{(n)}, e_{\tau}\right)\left(S^{* n+1-\gamma} T_{\psi}^{-1} S^{n+1-\gamma}\right)^{-1} e_{\tau} \\
& +\sum_{\tau=1}^{\gamma} \lambda_{n, \alpha, \tau}\left(S^{* n+1-\gamma} T_{\psi}^{-1} S^{n+1-\gamma}\right)^{-1} S^{\gamma} S^{* n+1-\gamma} T_{\psi}^{-1} S^{* \gamma} y_{\tau} .
\end{aligned}
$$


Taking the inner product on both sides with $S^{* n+1} y_{k}$ we get

$$
\begin{gathered}
\left(w_{\alpha}^{(n)}, S^{* n+1} y_{k}\right)=\sum_{\tau=1}^{\gamma}\left(S^{* n+1-\gamma} T_{\psi}^{-1} S^{n+1-\gamma} w_{\alpha}^{(n)}, e_{\tau}\right) \\
\cdot\left(\left[S^{* n+1-\gamma} T_{\psi}^{-1} S^{n+1-\gamma}\right]^{-1} e_{\tau}, S^{* n+1} y_{k}\right) \\
+\sum_{\ell=1}^{\gamma}\left(w_{\alpha}^{(n)}, S^{* n+1} y_{\ell}\right)\left(\left[S^{* n+1-\gamma} T_{\psi}^{-1} S^{n+1-\gamma}\right]^{-1} S^{\gamma} S^{* n+1} T_{\psi}^{-1} S^{* \gamma} y_{\ell}, S^{* n+1} y_{k}\right) .
\end{gathered}
$$

Now define $\gamma \times \gamma$ matrices $A_{n}, B_{n}, E_{n}, F_{n}$ as follows:

$$
\begin{gathered}
A_{n}=\left(\left(S^{n+1} w_{\alpha}^{(n)}, y_{\tau}\right)\right), \\
B_{n}=\left(\left(S^{* n+1-\gamma} T_{\psi}^{-1} S^{n+1-\gamma} w_{\alpha}^{(n)}, e_{\tau}\right)\right), \\
E_{n}=\left(\left(S^{n+1}\left(S^{* n+1-\gamma} T_{\psi}^{-1} S^{n+1-\gamma}\right)^{-1} e_{\alpha}, y_{\tau}\right)\right), \\
F_{n}=\left(\left(S^{n+1}\left(S^{* n+1-\gamma} T_{\psi}^{-1} S^{n+1-\gamma}\right)^{-1} \cdot S^{\gamma} S^{* n+1-\gamma} T_{\psi}^{-1} S^{* \gamma} y_{\alpha}, y_{\tau}\right) .\right.
\end{gathered}
$$

The preceding system of equations becomes $A_{n}=B_{n} E_{n}+A_{n} F_{n}$. Accordingly, $\operatorname{det} A_{n}=\frac{\operatorname{det} B_{n} \operatorname{det} E_{n}}{\operatorname{det}\left(1-F_{n}\right)}$. By Lemma 5.2, $B_{n}=\left(\left(T_{\overline{1}} e_{\alpha}, e_{\tau}\right)\right)+O\left(n^{-2 \beta}\right)$, and since $\left(T_{\psi}\right)^{-1}$ maps $\operatorname{Lip}_{\beta}$ to $\operatorname{Lip}_{\beta}$ we have $F_{n}=O\left(n^{-2 \beta}\right)$, so that

$$
\operatorname{det} A_{n}=\operatorname{det}\left(T_{\overline{\bar{g}}} e_{\alpha}, e_{\tau}\right) \cdot \operatorname{det} E_{n} \cdot\left[1+O\left(n^{-2 \beta}\right)\right]=\frac{1}{\bar{g}(0)^{\gamma}} \operatorname{det} E_{n}\left[1+O\left(n^{-2 \beta}\right)\right] .
$$

Therefore,

$\operatorname{det}\left(\left(S^{n+1} w_{\alpha}^{(n)}, y_{\tau}\right)\right)=\frac{1}{\bar{g}(0)^{\gamma}} \operatorname{det}\left(S^{n+1}\left(S^{* n+1-\gamma} T_{\psi}^{-1} S^{n+1-\gamma}\right)^{-1} e_{\alpha}, y_{\tau}\right) \cdot\left[1+O\left(n^{-2 \beta}\right)\right]$.

Lemma 5.6.

$$
\begin{aligned}
& \operatorname{det}\left(\left(S^{n+1}\left(S^{* n+1-\gamma} T_{\psi}^{-1} S^{n+1-\gamma}\right)^{-1} e_{\alpha}, T_{\frac{1}{g}} e_{\tau}\right)\right) \\
& \quad=\bar{g}(0)^{\gamma} \cdot \operatorname{det}\left(\left(T_{\frac{f}{g} z^{n+1}} \cdot\left[1-H_{\frac{\bar{g}}{f}} Q_{n-\gamma} H_{\left(\frac{f}{g}\right)}\right]^{-1} e_{\alpha}, e_{\tau}\right)\right)_{\gamma \times \gamma}
\end{aligned}
$$

Proof. We have

$$
\begin{aligned}
& S^{* n+1-\gamma} T_{\psi}^{-1} S^{n+1-\gamma}=S^{* n+1-\gamma} T_{f \bar{g}}^{-1} S^{n+1-\gamma}=S^{* n+1-\gamma} T_{\frac{1}{f}} T_{\frac{1}{g}} S^{n+1-\gamma} \\
& \quad=S^{* n+1-\gamma} T_{\frac{1}{\bar{g}}}\left\langle T_{\bar{g}}, T_{\frac{1}{f}}\right\rangle T_{\frac{1}{f}} S^{n+1-\gamma}=T_{\frac{1}{\bar{g}}} S^{* n+1-\gamma} T_{\frac{\bar{g}}{f}} T_{\frac{f}{\bar{g}}} S^{n+1-\gamma} T_{\frac{1}{f}} \\
& \quad=T_{\frac{1}{\bar{g}}}\left(1-S^{* n+1-\gamma} H_{\frac{\bar{g}}{f}} H_{\left(\frac{f}{\bar{g}}\right)} S^{n+1-\gamma}\right) T_{\frac{1}{f}}=T_{\frac{1}{\bar{g}}}\left[1-H_{\frac{\bar{g}}{f}} Q_{n-\gamma} H_{\left(\frac{f}{\bar{g}}\right)}\right] T_{\frac{1}{f}} .
\end{aligned}
$$

Thus

$$
\left[S^{* n+1-\gamma} T_{\psi}^{-1} S^{n+1-\gamma}\right]^{-1}=T_{f}\left[1-H_{\frac{\bar{g}}{f}} Q_{n-\gamma} H_{\left(\frac{f}{\bar{g}}\right)}\right]^{-1} T_{\bar{g}},
$$

and so,

$$
\begin{gathered}
\operatorname{det}\left(\left(S^{n+1}\left[S^{* n+1-\gamma} T_{\psi}^{-1} S^{n+1-\gamma}\right]^{-1} e_{\alpha}, T_{\frac{1}{g}} e_{\tau}\right)\right) \\
=\operatorname{det}\left(\left(T_{\frac{f}{g} z^{n+1}} \cdot\left[1-H_{\frac{\bar{g}}{f}} Q_{n-\gamma} H_{\left(\frac{f}{g}\right)}\right]^{-1} T_{\bar{g}} e_{\alpha}, e_{\tau}\right)\right)_{\gamma \times \gamma} \\
=\bar{g}(0)^{\gamma} \operatorname{det}\left(\left(T_{\frac{f}{g} z^{n+1}} \cdot\left[1-H_{\frac{\bar{g}}{f}} Q_{n-\gamma} H_{\left(\frac{f}{\bar{g}}\right)}\right]^{-1} e_{\alpha}, e_{\tau}\right)\right)_{\gamma \times \gamma} .
\end{gathered}
$$


Collecting the results: by equation (3.3) we have for $n \gg 0$,

$$
D_{n}\left(T_{\phi}\right)=\mathbf{G}(\phi)^{n+1} \frac{\left.\operatorname{det} P_{\mathcal{X}} X_{n} P_{\mathcal{X}}\right)}{\operatorname{det}\left(K_{n}\right)} \cdot \operatorname{det}\left(S^{n+1} v_{\alpha}^{(n)}, y_{\tau}\right) .
$$

By equation (5.1) we have

$$
\operatorname{det} P_{\mathcal{X}} X_{n} P_{\mathcal{X}}=\prod_{\lambda \in \sigma\left(T_{\phi} T_{\phi}-1\right) \backslash\{0\}} \lambda\left[1+O\left(n^{1-2 \beta}\right)\right],
$$

and by Lemmas 3.5 and 5.4

$$
\operatorname{det}\left(K_{n}\right)=(-1)^{\sum_{1}^{\gamma} m_{\alpha}} \operatorname{det}\left(b_{\alpha}, y_{\tau}\right) \cdot\left[1+O\left(n^{-2 \beta}\right)\right],
$$

while Lemmas 5.5 and 5.6 give us

$$
\begin{gathered}
\left(S^{n+1} v_{\alpha}^{(n)}, y_{\tau}\right)=\operatorname{det} \Gamma^{(n)} \cdot \operatorname{det}\left(S^{n+1} w_{\alpha}^{(n)}, y_{\tau}\right) \\
=\frac{1}{\left\|\bigwedge T_{f} e_{\alpha}\right\|} \operatorname{det}\left(S^{n+1} T_{f}\left[1-H_{\frac{\bar{g}}{f}} Q_{n-\gamma} H_{\left(\frac{f}{g}\right)}\right]^{-1} e_{\alpha}, y_{\tau}\right) \cdot\left[1+O\left(n^{-2 \beta}\right)\right] .
\end{gathered}
$$

On the other hand, Proposition 2.1 ii) gives

$$
\left\|\bigwedge T_{f} e_{\alpha}\right\| \operatorname{det}\left(b_{\alpha}, y_{\tau}\right)=\operatorname{det}\left(u_{\alpha}, y_{\tau}\right),
$$

and we have "the ratio invariance property"

$$
\frac{\operatorname{det}\left(S^{n+1} w_{\alpha}^{(n)}, y_{\tau}\right)}{\operatorname{det}\left(u_{\alpha}, y_{\tau}\right)}=\frac{\operatorname{det}\left(S^{n+1} w_{\alpha}^{(n)}, T_{\frac{1}{g}} e_{\tau}\right)}{\operatorname{det}\left(u_{\alpha}, T_{\frac{1}{g}} e_{\tau}\right)},
$$

while Theorem 1.2 gives us

$$
(-1)^{\sum_{\alpha=1}^{\gamma} m_{\alpha}} \frac{\prod_{\lambda \in \sigma\left(T_{\phi} T_{\phi^{-1}}\right) \backslash\{0\}} \lambda}{\operatorname{det}\left(u_{\alpha}, T_{\frac{1}{g}} e_{\alpha}\right)}=E(\psi) G\left(\frac{\bar{g}}{f}\right)^{\gamma} .
$$

Putting these pieces together yields the approximation theorem

$$
\begin{gathered}
D_{n}\left(T_{\phi}\right)=\mathbf{G}(\phi)^{n+1} E(\psi) G\left(\frac{\bar{g}}{f}\right)^{\gamma} \\
\cdot \operatorname{det}\left(\left(T_{\frac{f}{g} z^{n+1}} \cdot\left[1-H_{\frac{\bar{g}}{f}} Q_{n-\gamma} H_{\left(\frac{f}{g}\right)}\right]^{-1} e_{\alpha}, e_{\tau}\right)\right)_{\gamma \times \gamma}\left[1+O\left(n^{1-2 \beta}\right)\right] .
\end{gathered}
$$

Note that if $\phi=z^{\gamma} f \bar{g}$ where $f, g \in K_{2,2}^{\frac{1}{2}, \frac{1}{2}} \cap H^{\infty}(\mathbb{T})$, the same formula holds with $O\left(n^{-2 \beta}\right)$ replaced by $o(1)$. This concludes the proof of Theorem 1.3.

6. Rational Symbol $\phi$ With $D_{n}\left(T_{\phi}\right) \neq 0$ AND $D_{\gamma-1}\left(T_{\frac{f}{g}} z^{n+1}\right)=0$

Now we construct a rational symbol $\phi$ with $\phi \neq 0$ on $\mathbb{T}$ so that $T_{\phi}$ is injective and Fredholm and $D_{n}\left(T_{\phi}\right) \neq 0$ for $n \gg 0$, yet for an $r \neq 0$, we have the factorization $\operatorname{det}\left(P\left(T_{\bar{\phi}}\right) S^{n+1} P\left(T_{\phi^{-1}}\right) S^{* n+1} \mid \operatorname{ker} T_{\bar{\phi}}\right)=a_{n} r^{n+1}$ where $a_{n}$ is periodic in $n$, and one of the values it assumes is zero. Let $r \in(0,1), \theta \in(0, \pi)$. Set $\lambda_{1}=r e^{i \theta}, \lambda_{2}=$ $r e^{-i \theta}=\bar{\lambda}_{1} \neq \lambda_{1}, \mu=r=\left|\lambda_{1}\right| \neq \lambda_{1}, \lambda_{2}$. Set $\phi=\Theta_{\lambda_{1}} \Theta_{\lambda_{2}} \bar{\Theta}_{\mu}$. Then $\phi^{-1}=$ 
$\bar{\phi}, T_{\phi}$ is injective and Fredholm. Thus $\left.\operatorname{det}\left(P\left(T_{\bar{\phi}}\right) S^{n+1} P\left(T_{\phi^{-1}}\right) S^{* n+1}\right) \mid \operatorname{ker} T_{\bar{\phi}}\right)=$ $\left|\operatorname{det}\left(P\left(T_{\bar{\phi}}\right) S^{n+1} \mid \operatorname{ker} T_{\bar{\phi}}\right)\right|^{2}$. In Lemma 52 of [15] we showed that

$$
\begin{gathered}
\operatorname{det}\left(P\left(T_{\bar{\phi}}\right) S^{n+1} P\left(T_{\phi^{-1}}\right) \mid \operatorname{ker} T_{\bar{\phi}}\right)=\frac{1}{\left|1+r^{2} e^{i \theta}\right|^{2}+r^{2}\left|e^{i \theta}-1\right|^{2}} \frac{1}{\left(e^{-i \theta}-e^{i \theta}\right)} \\
\times\left[\left(e^{i \theta}-1\right)\left(1-r^{2} e^{i \theta}\right)\left(1-r^{2} e^{-2 i \theta}\right) e^{i(n+1) \theta}\right. \\
\left.-\left(e^{-i \theta}-1\right)\left(1-r^{2} e^{-i \theta}\right)\left(1-r^{2} e^{2 i \theta}\right) e^{-i(n+1) \theta}\right] r^{n+1} .
\end{gathered}
$$

Set

$$
B_{n}(\theta, r)=\left(e^{i \theta}-1\right)\left(1-r^{2} e^{i \theta}\right)\left(1-r^{2} e^{-2 i \theta}\right) e^{i(n+1) \theta},
$$

so that

$$
\begin{gathered}
\operatorname{det}\left(P\left(T_{\bar{\phi}}\right) S^{n+1} P\left(T_{\phi^{-1}}\right) \mid \operatorname{ker} T_{\bar{\phi}}\right) \\
=\frac{1}{\left|1+r^{2} e^{i \theta}\right|^{2}+r^{2}\left|e^{i \theta}-1\right|^{2}} \frac{r^{n+1}}{\left(e^{-i \theta}-e^{i \theta}\right)}\left[B_{n}(\theta, r)-B_{n}(-\theta, r)\right]=0,
\end{gathered}
$$

whenever $B_{n}(\theta, r)$ is real valued. Suppose that $n=0$. For fixed $\theta \in\left(0, \frac{\pi}{4}\right)$, consider $\Im B_{0}(\theta, r)$ as a function of $r \in[0,1]$. We have

$$
\begin{gathered}
\Im B_{0}(\theta, 0)=\sin 2 \theta-\sin \theta>0, \\
\Im B_{0}(\theta, 1)=-\left|1-e^{i \theta}\right|^{2} \sin 2 \theta<0 .
\end{gathered}
$$

Hence, by the intermediate value theorem, we get $r(\theta) \in(0,1)$ so that $\Im B_{0}(\theta, r(\theta))$ $=0$.

Now pick $\theta \in\left(0, \frac{\pi}{4}\right)$ so that $\frac{\theta}{\pi}$ is rational. Then with $r=r\left(\frac{\theta}{\pi}\right)$ the sequence $\left\{\Im B_{n}(\theta, r)\right\}_{n=1}^{\infty}$ is periodic with some period $P$ and has zero for one of its values. Hence $\operatorname{det}\left(P\left(T_{\bar{\phi}}\right) S^{k P+1} P\left(T_{\phi^{-1}}\right) \mid \operatorname{ker} T_{\bar{\phi}}\right)=0$ for $k=0,1,2, \ldots$

Now we must check that for the indicated choices of $r$ and $\theta$ the determinant $D_{n}\left(T_{\phi}\right) \neq 0$ for $n \gg 0$.

For this purpose we use results of K.M. Day [18,

Theorem 6.1. Let $\phi=\frac{c_{0} \prod_{j=1}^{p}\left(z-r_{j}\right)}{\prod_{j=1}^{h}\left(1-\frac{z}{\rho_{j}}\right) \prod_{j=1}^{\ell}\left(z-\delta_{j}\right)}$ on the circle $|z|=1$ with $\left|\delta_{j}\right|<$ $1(j=1, \ldots, \ell)$, and $\left|\rho_{j}\right|>1$ for $j=1, \ldots, h, c_{0}$ a constant and $r_{1}, \ldots, r_{p}$ pairwise distinct. Then,

$$
\text { For } p \geq \ell+h \text { and } n \geq 0, \quad D_{n}(\phi)=(-1)^{(p-\ell)(n+1)} \sum_{M} A_{M} r_{M}^{n+1}
$$

$$
\begin{gathered}
\text { for } n \geq \ell, \quad D_{n}(\phi)=0 \quad \text { if } p<\ell, \\
\text { and } D_{n}(\phi)=(-1)^{(p-\ell)(n+1)} \sum_{M} A_{M} r_{M}^{n+1} \quad \text { if } \quad p \geq \ell
\end{gathered}
$$


where the sum is taken over all $\left(\begin{array}{c}q \\ \ell\end{array}\right)$ subsets $M \subset\{1, \ldots, p\}$ of cardinality $\ell$, and with $\bar{M}=\{1, \ldots, p\} \backslash M$ the $r_{M}$ 's and the $A_{M}$ 's are given as

$$
\begin{gathered}
r_{M}=c_{0} \prod_{j \in \bar{M}} r_{j}, \\
A_{M}=\prod_{\substack{j \in \bar{M} \\
\alpha \in\{1, \ldots, \ell\}}}\left(r_{j}-\delta_{\alpha}\right) \prod_{\substack{i \in M \\
\beta \in\{1, \ldots, h\}}}\left(\rho_{\beta}-r_{i}\right) \prod_{\substack{\alpha \in\{1, \ldots, \ell\} \\
\beta \in\{1, \ldots, h\}}}\left(\rho_{\beta}-\delta_{\alpha}\right)^{-1} \prod_{\substack{i \in M \\
j \in M}}\left(r_{j}-r_{i}\right)^{-1} .
\end{gathered}
$$

In order to apply this theorem the $\phi$ of our example is rewritten as

$$
\phi=c_{0} \frac{\left(z-r_{1}\right)\left(z-r_{2}\right)\left(z-r_{3}\right)}{\left(1-\frac{z}{\rho_{1}}\right)\left(1-\frac{z}{\rho_{2}}\right)\left(z-\delta_{1}\right)}
$$

i.e.,

$$
c_{0}=-r, r_{1}=r e_{1}^{i \rho}=\frac{1}{r e^{i \theta}}, \rho_{2}=\frac{1}{r e^{-i \theta}}, \delta_{1}=r .
$$

Thus, $p=3, h=2$ and $\ell=1$. Accordingly, the set $M$ is either $\{1\},\{2\}$ or $\{3\}$ and we have

$$
\begin{aligned}
& A_{\{1\}} r_{\{1\}}{ }^{n+1}=\left(r_{2}-\delta_{1}\right)\left(r_{3}-\delta_{1}\right)\left(\rho_{1}-r_{1}\right)\left(\rho_{2}-r_{1}\right) \\
& \cdot\left(\rho_{1}-\delta_{1}\right)^{-1}\left(\rho_{2}-\delta_{1}\right)^{-1}\left(r_{2}-r_{1}\right)^{-1}\left(r_{3}-r_{1}\right)^{-1}(-r)^{n+1}\left(r_{2} r_{3}\right)^{n+1} . \\
& A_{\{2\}} r_{\{2\}}{ }^{n+1}=\left(r_{1}-\delta_{1}\right)\left(r_{3}-\delta_{1}\right)\left(\rho_{1}-r_{2}\right)\left(\rho_{2}-r_{2}\right) \cdot\left(\rho_{1}-\delta_{1}\right)^{-1}\left(\rho_{2}-\delta_{1}\right)^{-1}\left(r_{1}-r_{2}\right)^{-1}\left(r_{3}-r_{2}\right)^{-1}(-r)^{n+1}\left(r_{1} r_{3}\right)^{n+1} . \\
& A_{\{3\}} r_{\{3\}}{ }^{n+1}=\left(r_{1}-\delta_{1}\right)\left(r_{2}-\delta_{1}\right)\left(\rho_{1}-r_{3}\right)\left(\rho_{2}-r_{3}\right) \\
& \quad \cdot\left(\rho_{1}-\delta_{1}\right)^{-1}\left(\rho_{2}-\delta_{1}\right)^{-1}\left(r_{1}-r_{3}\right)^{-1}\left(r_{2}-r_{3}\right)^{-1}(-r)^{n+1}\left(r_{1} r_{2}\right)^{n+1} .
\end{aligned}
$$

A straightforward substitution in the Day formula now gives

$$
\begin{gathered}
A_{\{1\}} r_{\{1\}}^{n+1}+A_{\{2\}} r_{\{2\}}^{n+1}=\left(r_{3}-\delta_{1}\right)\left(\rho_{1}-\delta_{1}\right)^{-1}\left(\rho_{2}-\delta_{1}\right)^{-1}\left(r_{1}-r_{2}\right)^{-1}(-r)^{n+1} \\
\cdot \frac{\left(1-r^{2}\right)}{\left|1-r^{2} e^{i \theta}\right|^{2}} \cdot\left[\left(e^{i \theta}-1\right)\left(1-r^{2} e^{i \theta}\right)\left(1-r^{2} e^{-2 i \theta}\right) e^{i(n+1) \theta}\right. \\
\left.-\left(e^{-i \theta}-1\right)\left(1-r^{2} e^{-i \theta}\right)\left(1-r^{2} e^{2 i \theta}\right) e^{i(n+1) \theta}\right] \\
=\left(r_{3}-\delta_{1}\right)\left(\rho_{1}-\delta_{1}\right)^{-1}\left(\rho_{2}-\delta_{1}\right)^{-1}\left(r_{1}-r_{2}\right)^{-1}(-r)^{n+1} \\
2 \cdot \frac{\left(1-r^{2}\right)}{\left|1-r^{2} e^{i \theta}\right|^{2}} \Im B_{n}(\theta, r) .
\end{gathered}
$$

Hence there are non-zero constants $C_{1}$ and $C_{2}$ so that $D_{n}\left(T_{\phi}\right)$ has the form

$$
D_{n}\left(T_{\phi}\right)=\left[C_{1} \Im B_{n}(\theta, r)+C_{2} r^{2(n+1)}\right](-r)^{n+1} .
$$

Since $0<r<1$ and $\Im B_{n}(\theta, r)$ assumes only finitely many values, it follows that $D_{n}\left(T_{\phi}\right) \neq 0$ provided that $n \gg 0$.

\section{REFERENCES}

[1] J. Anderson and L.N. Vasserstein, Commutators in ideals of trace class operators, Indiana Univ. Mat. J. 35 (1986), 345-372. MR0833399 (87f:47025a)

[2] _ Commutators in ideals of trace class operators, II, Indiana Univ. Mat. J. 35 ( 1986) 373-382. MR0833400 (87f:47025b) 
[3] E.L. Basor, Asymptotic formulas for Toeplitz determinants, Trans. Amer. Math. Soc. 239 (1978), 33-65. MR0493480 (58:12484)

[4] E.L. Basor and J.W. Helton, A new proof of the Szegö limit theorem and new results for Toeplitz operators with discontinuous symbols, J. Operator Theory 3 (1980) 23-29. MR0565749 (81m:47042)

[5] G.A. Baxter, A convergence equivalence related to polynomials on the unit circle, Trans. Amer. Math. Soc. 99 (1961), 471-487. MR0126126 (23:A3422)

[6] _ A norm inequality for a "finite section" Wiener-Hopf equation, Illinois J. Math. 7 (1963), 97-103. MR0145285 (26:2818)

[7] A.A. Beilinson, Current Problems in Mathematics 24 (Russian) Akad. Nauk SSSR, Vsessoyuz, Inst. Nauchin. i Tekhn. Inform., Moscow (1984). MR0760999 (86h:11103)

[8] Higher regulators and values of L-functions of curves, Funktsional. Anal. i Prilozhen 14 No. 2,(1980) 46-47. MR 0575206 (81k:14020)

[9] A. Borodin and A. Okunkov, A Fredholm determinant formula for Toeplitz determinants, Integral Equations Operator Theory $\mathbf{3 7}$ (2000), 386-396. MR1780118(2001g:47042a)

[10] A. Böttcher and B. Silbermann, The asymptotic behaviour of Toeplitz determinants for generating functions with zeros of integral orders, Math. Nachr., 102 (1981), 79-105. MR 0642143 (83f:47022)

[11] - Invertibility and asymptotics of Toeplitz matrices, Math. Forschung 17, AkademieVerlag, Berlin, 1983. MR0734173 (85g:47037)

[12] _ Introduction to large truncated Toeplitz matrices, Springer-Verlag, Berlin, 1999. MR.1724795 (2001b:47043)

[13] Jean-Luc Brylinski, Loop spaces, characteristic classses, and geometric quantization, Progress in Mathematics, 3, Birkhäuser Boston, 1993. MR1197353 (94b:57030)

[14] R.W. Carey and J.D. Pincus, Joint Torsion, preprint (1995-1996), 1-174.

[15] — Perturbation Vectors, Integral Equations Operator Theory 35 (1999), 271-365. MR1716541(2001e:47048)

[16] - Joint torsion of Toeplitz operators with $\mathrm{H}^{\infty}$ symbols Integral Equations Operator Theory 33 (1999), 273-304. MR.1671481 (2000f:47050)

[17] _ Toeplitz operators with rational symbols, reciprocity, Integral Equations Operator Theory 40 (2001), 127-184. MR.1831825 (2002e:47030)

[18] K.M. Day, Toeplitz matrices generated by by the Laurent series expansion of an arbitrary rational function, Trans. Amer. Math. Soc. 206 ( 1975), 224-245. MR0379803 (52:708)

[19] L. Deligne, Le symbol moderé, Publ. Math., I.H.E.S. 173,(1991), 147-181. MR.1114212 (93i:14030)

[20] A. Devinatz, The strong Szegö limit theorem, Illinois. J. Math. 11 (1967), 160-175. MR0206620 (34:6438)

[21] M.E. Fisher and R.E. Hartwig, Toeplitz determinants, some applications, theorems and conjectures, 'J. Adv. Chem. Phys. 15, (1968), 333-353.

[22] _ Asymptotic behavior of Toeplitz matrices and determinants, J. Arch. Rational. Mech. Anal. 32, (1969), 190-255. MR0236593(38:4888)

[23] I.C. Gohberg and I.A. Fel'dman, Convolution equations and projection methods for their solution, Trans. of Math. Monographs Volume 41, translated from the Russian, American Mathematical Society, Providence, 1974. MR0355675 (50:8149)

[24] U. Grenander and G. Szegö, Toeplitz forms and their applications, Univ. of Calif. Press, Berkeley, 1958. MR0094840 (20:1349)

[25] I.I. Hirschman, The strong Szegö limit theorem for Toeplitz determinants, Amer. J. Math. 88 (1966), 577-614. MR0211182(35:2064)

[26] I.A. Ibragimov, On a theorem of G. Szegö, Mat. Zametki 3 (1968), 693-702. MR0231114 $(37: 6669)$

[27] Y. Katznelson, Introduction to Harmonic Analysis Wiley, New York, 1968. MR0248482 $(40: 1734)$

[28] M. Kac, Toeplitz matrices, translation kernels and a related problem in probability, Duke Math. J 21 (1954) 501-509. MR0062867(16:31a)

[29] G. Szegö, Ein Grenzwertsatz über die Toeplitzschen Determinanten einer reelen positiven Funktion, Math. Ann. 76 (1915), 490-503.

[30] On certain Hermitian forms associated with the Fourier series of a positive function, Festskrift Marcel Riesz, Lund (1952), 222-238. 
[31] H. Widom, Asymptotic behavior of block Toeplitz matrices and determinants II., Adv. in Math. 21 (1976), 1-29. MR0409512 (53:13266b)

[32] _ Eigenvalue distribution of non-self adjoint Toeplitz matrices and the asymptotics of Toeplitz determinants in the case of non-vanishing index, Operator Theory: Adv. and Appl. 48 (1990), 387-421. MR:1207410 (93m:47033)

Department of Mathematics, University of Kentucky, Lexington, Kentucky 40511

E-mail address: carey@ms.uky.edu

806 Hunt Lane, Manhasset, New York 11030

E-mail address: joelppp@earthlink.net 